Supporting Information for

\title{
Convergent Synthesis of Diverse Nitrogen Heterocycles via Rh(III)-Catalyzed C-H Conjugate Addition/Cyclization Reactions
}

\author{
Adam B. Weinstein and Jonathan A. Ellman* \\ Yale University Department of Chemistry \\ 225 Prospect Street, New Haven, CT, 06520-8107 \\ jonathan.ellman@yale.edu
}

\section{$\underline{\text { Table of Contents }}$}

I. General considerations

S2

II. Preparation of $\mathrm{C}-\mathrm{H}$ functionalization substrates

$\mathrm{S} 3-\mathrm{S} 4$

III. Preparation of electrophilic olefin coupling partners

S5 - S8

IV. Rhodium catalyzed coupling reactions

S9-S16

V. Synthesis of oxoassoanine

S17 - S21

VI. References

S22

VII. ${ }^{1} \mathrm{H}$ and ${ }^{13} \mathrm{C}$ NMR spectra for new compounds

$\mathrm{S} 23-\mathrm{S} 46$ 


\section{General considerations}

All commercially available reagents were purchased and used as received, unless otherwise noted. Reactions carried out in toluene, dichloromethane, diethyl ether, tetrahydrofuran or dioxane made use of solvent that was purified by elution through a column of activated alumina under argon. 1,2Dichloroethane (DCE) was deoxygenated by sparging with nitrogen gas and stored over activated $3 \AA$ molecular sieves in a nitrogen filled glovebox. Catalytic reactions were set up inside a nitrogen filled glovebox using oven-dried glassware, unless otherwise noted. Heating/stirring, work-up and isolation of products from catalytic reactions were performed on the benchtop using standard techniques. $\left[\mathrm{Cp}^{*} \mathrm{RhCl}_{2}\right]_{2}$ was prepared according to a published literature procedure ${ }^{1}$ and stored in a nitrogen filled glovebox, along with commercial $\mathrm{AgSbF}_{6} .{ }^{1} \mathrm{H}$ and ${ }^{13} \mathrm{C}$ NMR spectra were recorded on $400 \mathrm{MHz}, 500 \mathrm{MHz}$ or $600 \mathrm{MHz}$ spectrometers and chemical shifts are reported in parts per million relative to $\mathrm{CDCl}_{3}\left(7.26 \mathrm{ppm}\right.$ for ${ }^{1} \mathrm{H}$ NMR and 77.16 ppm for ${ }^{13} \mathrm{C}$ NMR). Flash chromatography was carried out with SiliaFlash ${ }^{\circledR}$ P60 (particle size 40-63 um, 230-400 mesh). Only partial data are provided for IR spectra. Melting points are reported uncorrected. High-resolution mass spectra (HRMS) were recorded on an Agilent 6550 iFunnel QTOF LCMS. 


\section{Preparation of $\mathbf{C}-\mathbf{H}$ functionalization substrates}

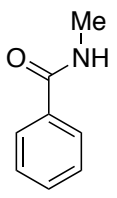

$1 \mathrm{a}$

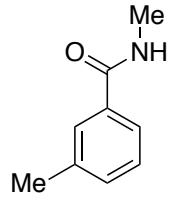

$1 \mathrm{~b}$
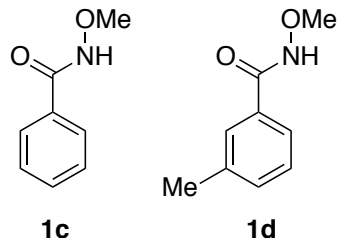

1d

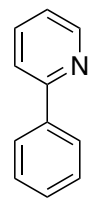

1e

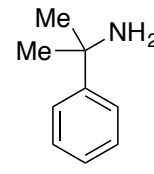

$1 f$

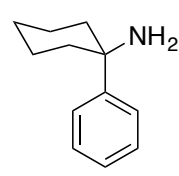

$1 \mathrm{~g}$

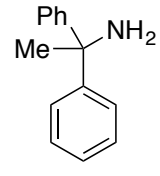

$1 \mathrm{~h}$

$\mathrm{N}$-Methyl benzamide (1a), 2-phenylpyridine (1e) and cumylamine (1f) were purchased from commercial sources and used as received. $N$-Methyl 3-methylbenzamide (1b), ${ }^{2} N$-methoxy benzamide $(\mathbf{1 c})^{3}$ and $N$ methoxy 3-methylbenzamide (1d) ${ }^{3}$ were prepared according to prior reports.

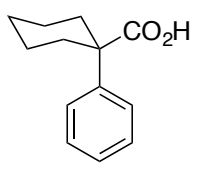

1 equiv

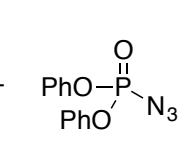

1.1 equiv

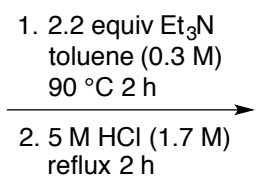

reflux $2 \mathrm{~h}$

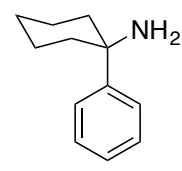

$1 \mathrm{~g}$

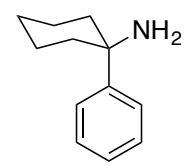

1-Phenylcyclohexan-1-amine (1g): 1-phenylcyclohexanecarboxylic acid (1.02 g, 5.00 mmol, 1 equiv), was weighed into a $100 \mathrm{ml}$ round bottomed flask equipped with a stir bar, and the reaction vessel was flushed with nitrogen. Toluene $(17 \mathrm{~mL})$ and triethylamine $(1.52$ $\mathrm{mL}, 11.0 \mathrm{mmol}, 2.2$ equiv) were added via syringe. Diphenylphosphoryl azide (1.18 mL, $5.50 \mathrm{mmol}, 1.1$ equiv) was added via syringe. The flask was equipped with a reflux condenser, and the reaction mixture was heated to $90{ }^{\circ} \mathrm{C}$. Gas evolution was notable upon heating. After 2 hours, the reaction mixture was cooled to room temperature, diluted with ethyl acetate and transferred to a separatory funnel. The organic layer was washed with saturated sodium bicarbonate solution $2 \mathrm{x}$ and then with brine. The organic layer was dried over magnesium sulfate and concentrated to an oil. The residue was transferred to a $25 \mathrm{~mL}$ round bottomed flask equipped with a stir bar, and the residue was suspended in $5 \mathrm{M} \mathrm{HCl}(3 \mathrm{~mL})$. A reflux condenser was attached to the flask and the mixture was heated to reflux, with notable evolution of gas. The mixture was cooled to room temperature and transferred in toluene to a $100 \mathrm{~mL}$ round bottomed flask. The solvent was removed under vacuum, including azeotropic removal of water, leaving a white solid. The white solid was dissolved and partitioned between $100 \mathrm{~mL}$ of saturated sodium bicarbonate solution and $100 \mathrm{~mL}$ of ethyl acetate. The organic layer was washed $4 \mathrm{x}$ with saturated sodium bicarbonate solution, then dried over magnesium sulfate and concentrated to an oil. Purification over silica using a 3:2 hexanes:ethyl acetate / $2 \%$ triethylamine solvent system afforded the desired product as a clear oil (574 $\mathrm{mg}, 66 \%$ yield). Characterization data was consistent with previously reported data. ${ }^{4} \mathrm{H}$ NMR (400 MHz, $\left.\mathrm{CDCl}_{3}\right) \delta 7.56-7.50(\mathrm{~m}, 2 \mathrm{H}), 7.35(\mathrm{t}, J=7.7 \mathrm{~Hz}, 2 \mathrm{H}), 7.25-7.19(\mathrm{~m}, 1 \mathrm{H}), 2.02-1.92(\mathrm{~m}, 2 \mathrm{H}), 1.73-$ $1.46(\mathrm{~m}, 9 \mathrm{H}), 1.40-1.28(\mathrm{~m}, 1 \mathrm{H})$. 


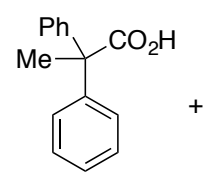

1 equiv

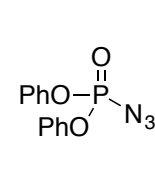

1.1 equiv

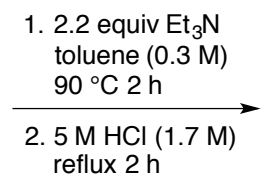

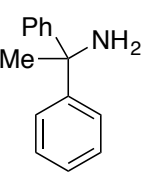

$1 \mathrm{~h}$

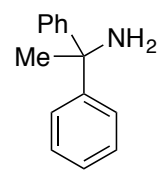

1,1-Diphenylethan-1-amine (1h): 2,2-diphenylpropanoic acid (1.13 g, $5.00 \mathrm{mmol}, 1$ equiv), was weighed into a $100 \mathrm{~mL}$ round bottomed flask equipped with a stir bar, and the reaction vessel was flushed with nitrogen. Toluene $(17 \mathrm{~mL})$ and triethylamine $(1.52 \mathrm{~mL}, 11.0 \mathrm{mmol}$, via syringe. The flask was equipped with a reflux condenser, and the reaction mixture was heated to 90 ${ }^{\circ} \mathrm{C}$. Gas evolution was notable upon heating. After 2 hours, the reaction mixture was cooled to room temperature, diluted with ethyl acetate and transferred to a separatory funnel. The organic layer was washed with saturated sodium bicarbonate solution $2 \mathrm{x}$ and then with brine. The organic layer was dried over magnesium sulfate and concentrated to an oil. The residue was transferred to a $25 \mathrm{~mL}$ round bottomed flask equipped with a stir bar, and the residue was suspended in $5 \mathrm{M} \mathrm{HCl}(3 \mathrm{~mL})$. A reflux condenser was affixed to the flask, and the mixture was heated to reflux, with notable evolution of gas. The mixture was cooled to room temperature and transferred in toluene to a $100 \mathrm{~mL}$ round bottomed flask. The solvent was removed under vacuum, including azeotropic removal of water, leaving a white solid. The white solid was dissolved and partitioned between $100 \mathrm{~mL}$ of saturated sodium bicarbonate solution and $100 \mathrm{~mL}$ of ethyl acetate. The organic layer was washed $4 \mathrm{x}$ with saturated sodium bicarbonate solution, then dried over magnesium sulfate and concentrated to an oil. Purification over silica using a 65:35 hexanes:ethyl acetate / $2 \%$ triethylamine solvent system afforded the desired product as a clear oil (820 mg, 83\% yield). ${ }^{1} \mathrm{H}$ NMR $\left(600 \mathrm{MHz}, \mathrm{CDCl}_{3}\right) \delta 7.38(\mathrm{~d}, J=6.9 \mathrm{~Hz}, 4 \mathrm{H}), 7.30(\mathrm{t}, J=7.7 \mathrm{~Hz}, 4 \mathrm{H})$, $7.22(\mathrm{t}, J=7.3 \mathrm{~Hz}, 2 \mathrm{H}), 1.86(\mathrm{~s}, 3 \mathrm{H}), 1.85$ (br s, 2H); ${ }^{13} \mathrm{C}$ NMR $\left(151 \mathrm{MHz}, \mathrm{CDCl}_{3}\right) \delta 149.99,128.25$, 126.47, 126.28, 58.55, 32.00. IR (neat) $\mathrm{cm}^{-1}: 3060,1597,1491,1445,1027$. HRMS (ESI) calculated for $\left[\mathrm{C}_{14} \mathrm{H}_{13}\right]^{+}$(loss of ammonia) requires $m / z$ 181.1012, found 181.1014 . 


\section{Preparation of electrophilic olefin coupling partners}

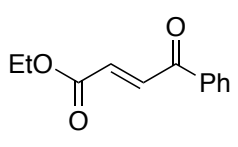

$2 a$

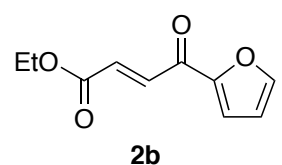

$2 b$

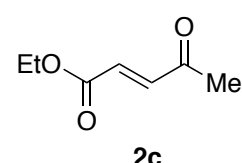

2c

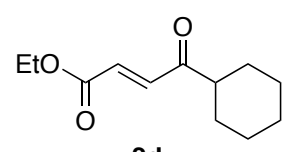

2d

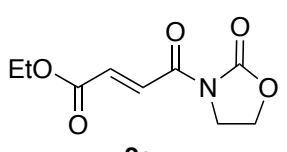

$2 \mathrm{e}$

Ethyl (E)-4-oxo-4-phenylbut-2-enoate (2a) was purchased from commercial sources and purified over silica (pentane/ether) prior to use.

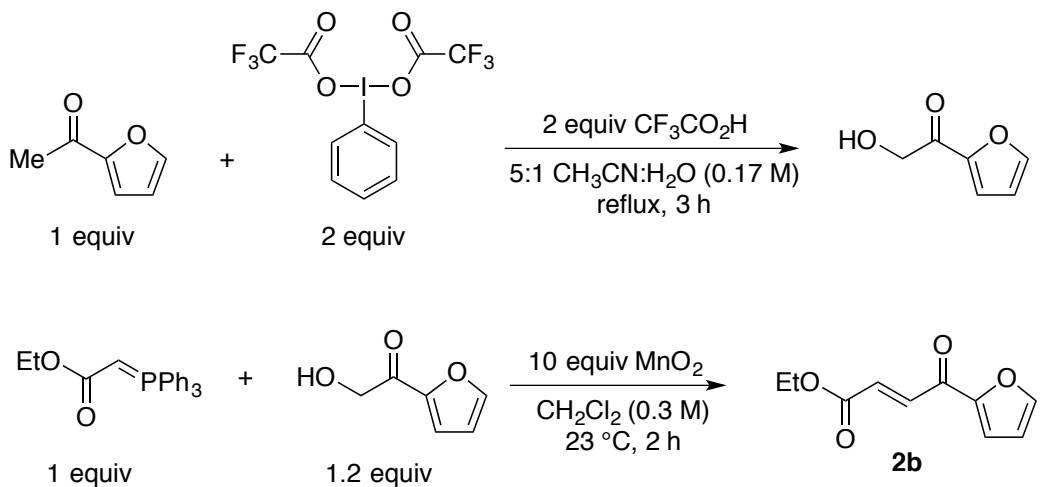

Ethyl $(E)$-4-(furan-2-yl)-4-oxobut-2-enoate (2b): According to a literature procedure, ${ }^{5}$
2-acetylfuran $(1.10 \mathrm{~g}, 10.0 \mathrm{mmol}, 1$ equiv) was weighed into a $250 \mathrm{~mL}$ round bottomed flask with a stir bar. A solution of trifluoroacetic acid $(1.49 \mathrm{~mL}, 20.0 \mathrm{mmol}, 2$ equiv) in $50 \mathrm{~mL}$ of acetonitrile and $10 \mathrm{~mL}$ of water was added to the reaction vessel. Bis(trifluoroacetoxy)iodobenzene ( $8.60 \mathrm{~g}, 20.0 \mathrm{mmol}, 2$ equiv) was added in one portion. The flask was equipped with a reflux condenser, and the reaction mixture was heated to reflux for 3 hours. The reaction mixture was then cooled to room temperature and concentrated to remove the acetonitrile. The residue was partitioned between dichloromethane $(200 \mathrm{~mL})$ and water $(100 \mathrm{~mL})$, and the aqueous phase was re-extracted $3 \mathrm{x}$ with dichloromethane $(50 \mathrm{~mL})$. The combined organics were washed with saturated sodium bicarbonate $3 \mathrm{x}$, then dried over magnesium sulfate and concentrated to a white solid. The solid residue was lixiviated with cold hexanes to dissolve the iodobenzene, leaving the desired product as a white solid (537 $\mathrm{mg}, 43 \%$ yield). ${ }^{1} \mathrm{H}$ NMR $\left(500 \mathrm{MHz}, \mathrm{CDCl}_{3}\right) \delta 7.63(\mathrm{~d}, J=1.6 \mathrm{~Hz}, 1 \mathrm{H}), 7.30(\mathrm{~d}, J=3.6 \mathrm{~Hz}, 1 \mathrm{H}), 6.60(\mathrm{dd}, J=3.6$, $1.7 \mathrm{~Hz}, 1 \mathrm{H}), 4.74$ (d, $J=4.6 \mathrm{~Hz}, 2 \mathrm{H}), 3.23$ (t, $J=4.9 \mathrm{~Hz}, 1 \mathrm{H}) .1$-(Furan-2-yl)-2-hydroxyethan-1-one (537 $\mathrm{mg}, 4.26 \mathrm{mmol}, 1.2$ equiv) thus obtained was transferred into a $100 \mathrm{~mL}$ round bottomed flask equipped with a stir bar. In accordance with a literature procedure, ${ }^{6}$ dichloromethane $(12 \mathrm{~mL})$ was added via syringe, followed by ethyl 2-(triphenyl- $\lambda^{5}$-phosphanylidene)acetate (1.24 g, $3.55 \mathrm{mmol}$, 1 equiv). Manganese dioxide (3.09 g, $35.5 \mathrm{mmol}, 10$ equiv) was added in one portion with vigorous stirring, and an exothermic reaction was noticeable. The reaction mixture was stirred at room temperature for 2 hours, 
then filtered over celite and concentrated. The solid residue was dissolved in a minimal amount of dichloromethane, loaded onto a plug of silica, and the crude product was eluted with a 1:1 pentane:diethyl ether solvent mixture to afford a yellow solid after concentration. Purification over silica using a 3:1 pentane:diethyl ether solvent system afforded the product as a pale yellow solid (565 $\mathrm{mg}, 82 \%$ yield, m.p. $\left.=59-64{ }^{\circ} \mathrm{C}\right) .{ }^{1} \mathrm{H} \mathrm{NMR}\left(600 \mathrm{MHz}, \mathrm{CDCl}_{3}\right) \delta 7.75(\mathrm{~d}, J=15.6 \mathrm{~Hz}, 1 \mathrm{H}), 7.69(\mathrm{~s}, 1 \mathrm{H}), 7.37(\mathrm{dd}, J=3.7,0.8$ $\mathrm{Hz}, 1 \mathrm{H}), 6.97(\mathrm{~d}, J=15.6 \mathrm{~Hz}, 1 \mathrm{H}), 6.62(\mathrm{dd}, J=3.6,1.6 \mathrm{~Hz}, 1 \mathrm{H}), 4.29$ (q, $J=7.1 \mathrm{~Hz}, 2 \mathrm{H}), 1.34(\mathrm{t}, J=7.2$ $\mathrm{Hz}, 3 \mathrm{H}) ;{ }^{13} \mathrm{C} \mathrm{NMR}\left(151 \mathrm{MHz}, \mathrm{CDCl}_{3}\right) \delta 176.67,165.59,152.94,147.94,135.60,132.35,119.68,113.06$, $61.55,14.30$. IR (neat) $\mathrm{cm}^{-1}: 3131,1705,1665,1628,1297,1168,987$. HRMS (ESI) calculated for $\left[\mathrm{C}_{10} \mathrm{H}_{10} \mathrm{O}_{4} \mathrm{Na}\right]^{+}$requires $\mathrm{m} / z$ 217.0471, found 217.0475.
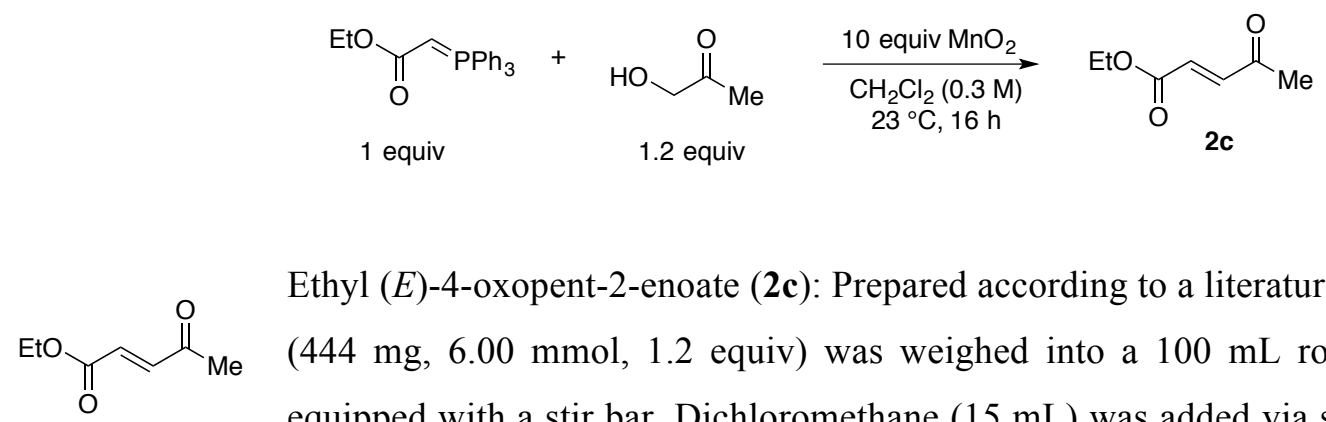

Ethyl (E)-4-oxopent-2-enoate (2c): Prepared according to a literature procedure. ${ }^{6}$ Acetol (444 mg, $6.00 \mathrm{mmol}, 1.2$ equiv) was weighed into a $100 \mathrm{~mL}$ round bottomed flask equipped with a stir bar. Dichloromethane $(15 \mathrm{~mL})$ was added via syringe, followed by ethyl 2-(triphenyl- $\lambda^{5}$-phosphanylidene)acetate (1.74 g, $5.00 \mathrm{mmol}, 1$ equiv). Manganese dioxide (4.35 g, $50.0 \mathrm{mmol}, 10$ equiv) was added in one portion with vigorous stirring, and an exothermic reaction was noticeable. The reaction mixture was stirred at room temperature for 16 hours, then filtered over celite and concentrated. The solid residue was dissolved in a minimal amount of dichloromethane, loaded onto a plug of silica, and the crude product was eluted with a 1:1 pentane:diethyl ether solvent mixture to afford a yellow liquid after concentration. Purification over silica using a 4:1 pentane:diethyl ether solvent system afforded the product as a pale yellow liquid (380 mg, 54\% yield). Characterization data was consistent with previously reported data. ${ }^{7} \mathrm{H}$ NMR $\left(500 \mathrm{MHz}, \mathrm{CDCl}_{3}\right) \delta 7.01(\mathrm{~d}, J=16.1 \mathrm{~Hz}, 1 \mathrm{H}), 6.64$ $(\mathrm{d}, J=16.1 \mathrm{~Hz}, 1 \mathrm{H}), 4.27$ (q, $J=7.1 \mathrm{~Hz}, 2 \mathrm{H}), 2.36$ (s, 3H), 1.32 (t, $J=7.1 \mathrm{~Hz}, 3 \mathrm{H})$. 

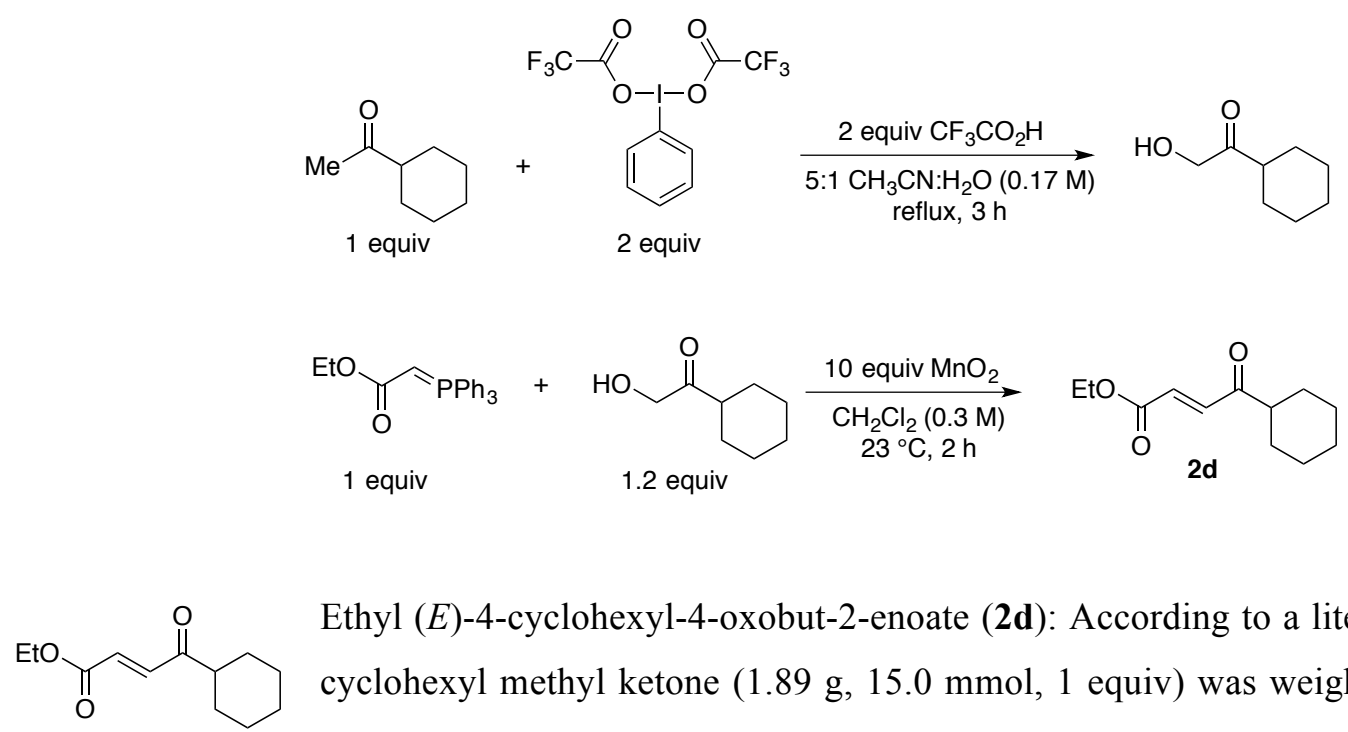

Ethyl (E)-4-cyclohexyl-4-oxobut-2-enoate (2d): According to a literature procedure, ${ }^{5}$ cyclohexyl methyl ketone $(1.89 \mathrm{~g}, 15.0 \mathrm{mmol}, 1$ equiv) was weighed into a $250 \mathrm{~mL}$ round bottomed flask with a stir bar. A solution of trifluoroacetic acid $(2.23 \mathrm{~mL}, 30.0$ mmol, 2 equiv) in $75 \mathrm{~mL}$ acetonitrile and $15 \mathrm{~mL}$ water was added to the reaction vessel. Bis(trifluoroacetoxy)iodobenzene (12.9 g, $30.0 \mathrm{mmol}, 2$ equiv) was added in one portion. The flask was equipped with a reflux condenser, and the reaction mixture was heated to reflux for 3 hours. The reaction mixture was then cooled to room temperature and concentrated to remove the acetonitrile. The residue was partitioned between dichloromethane $(200 \mathrm{~mL})$ and water $(100 \mathrm{~mL})$, and the aqueous phase was reextracted $3 \mathrm{x}$ with dichloromethane $(50 \mathrm{~mL})$. The combined organics were washed with saturated sodium bicarbonate $3 \mathrm{x}$, then dried over magnesium sulfate and concentrated to a yellow liquid. The residue was purified over silica using a 1:1 pentane:diethyl ether solvent system to afford the product as a pale yellow liquid (860 mg, 40\% yield). ${ }^{1} \mathrm{H}$ NMR (400 MHz, $\left.\mathrm{CDCl}_{3}\right) \delta 4.30$ (s, 2H), 3.14 (br s, $\left.1 \mathrm{H}\right), 2.38$ (tt, $J=11.6$, $3.4 \mathrm{~Hz}, 1 \mathrm{H}), 1.88-1.76(\mathrm{~m}, 4 \mathrm{H}), 1.72-1.64(\mathrm{~m}, 1 \mathrm{H}), 1.47-1.36(\mathrm{~m}, 2 \mathrm{H}), 1.35-1.22(\mathrm{~m}, 3 \mathrm{H})$. $1-$ (Cyclohexyl)-2-hydroxyethan-1-one ( $857 \mathrm{mg}, 6.03 \mathrm{mmol}, 1.2$ equiv) thus obtained was transferred into a $100 \mathrm{~mL}$ round bottomed flask equipped with a stir bar. In accordance with a literature procedure, ${ }^{6}$ dichloromethane $(15 \mathrm{~mL})$ was added via syringe, followed by ethyl 2-(triphenyl-1 ${ }^{5}$ phosphanylidene)acetate (1.75 g, $5.02 \mathrm{mmol}, 1$ equiv). Manganese dioxide (4.37 g, $50.2 \mathrm{mmol}, 10$ equiv) was added in one portion with vigorous stirring, and an exothermic reaction was noticeable. The reaction mixture was stirred at room temperature for 2 hours, then filtered over celite and concentrated. The solid residue was dissolved in a minimal amount of dichloromethane, loaded onto a plug of silica, and the crude product was eluted with a 1:1 pentane:diethyl ether solvent mixture to afford a yellow oil after concentration. Purification over silica using a 9:1 pentane:diethyl ether solvent system afforded the product as a yellow oil $\left(770 \mathrm{mg}, 73 \%\right.$ yield). ${ }^{1} \mathrm{H} \mathrm{NMR}\left(600 \mathrm{MHz}, \mathrm{CDCl}_{3}\right) \delta 7.16(\mathrm{~d}, J=15.8 \mathrm{~Hz}, 1 \mathrm{H})$, $6.69(\mathrm{~d}, J=15.8 \mathrm{~Hz}, 1 \mathrm{H}), 4.26(\mathrm{q}, J=7.1 \mathrm{~Hz}, 2 \mathrm{H}), 2.59$ (tt, $J=11.2,3.4 \mathrm{~Hz}, 1 \mathrm{H}), 1.91-1.85(\mathrm{~m}, 2 \mathrm{H})$, $1.81(\mathrm{dt}, J=12.6,3.3 \mathrm{~Hz}, 2 \mathrm{H}), 1.73-1.66(\mathrm{~m}, 1 \mathrm{H}), 1.41-1.27(\mathrm{~m}, 4 \mathrm{H}), 1.32(\mathrm{t}, J=7.1 \mathrm{~Hz}, 3 \mathrm{H}), 1.21$ 
(qt, $J=12.1,3.4 \mathrm{~Hz}, 1 \mathrm{H}) ;{ }^{13} \mathrm{C}$ NMR $\left(151 \mathrm{MHz}, \mathrm{CDCl}_{3}\right) \delta 202.52,165.81,138.42,130.81,61.47,49.86$, 28.26, 25.88, 25.65, 14.29. IR (neat) $\mathrm{cm}^{-1}: 2931,2855,1722,1696,1277,1179$. HRMS (ESI) calculated for $\left[\mathrm{C}_{12} \mathrm{H}_{18} \mathrm{O}_{3} \mathrm{Na}\right]^{+}$requires $m / z 233.1148$, found 233.1150 .
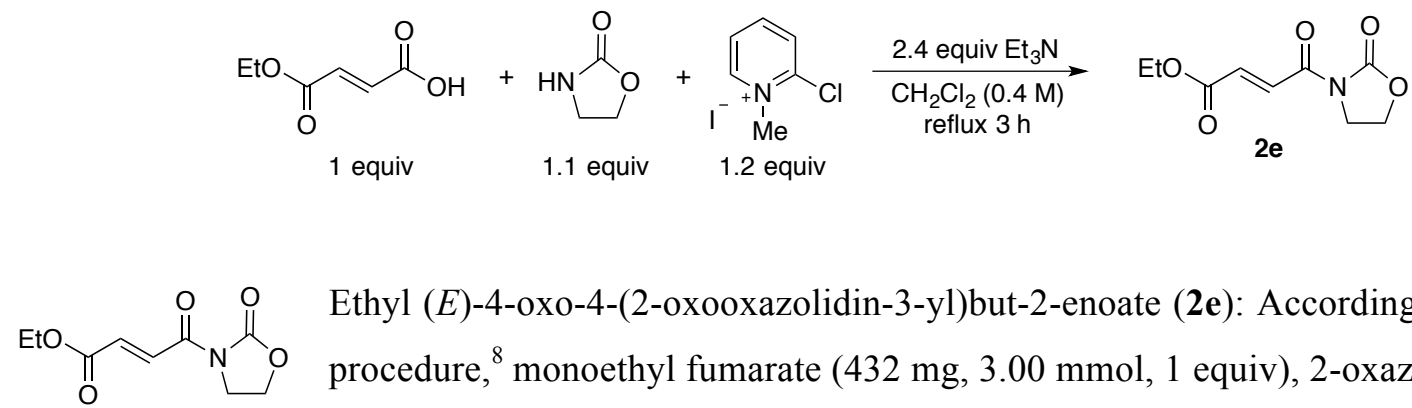

Ethyl (E)-4-oxo-4-(2-oxooxazolidin-3-yl)but-2-enoate (2e): According to a literature procedure, ${ }^{8}$ monoethyl fumarate (432 $\mathrm{mg}, 3.00 \mathrm{mmol}, 1$ equiv), 2-oxazolidinone (287 $\mathrm{mg}, 3.30 \mathrm{mmol}, 1.1$ equiv) and 2-chloro-1-methylpyridinium iodide (920 mg, 3.60 mmol, 1.2 equiv) were combined in a $25 \mathrm{~mL}$ pear shaped flask with a stir bar. Dichloromethane $(7.5 \mathrm{~mL})$ and triethylamine (1.00 mL, $7.20 \mathrm{mmol}, 2.4$ equiv) were added via syringe. The flask was equipped with a reflux condenser, and the reaction mixture was heated to reflux for 3 hours. The reaction mixture was cooled to room temperature, concentrated and purified over silica using a 1:1 hexanes:ethyl acetate solvent system to afford the product as a white solid (588 mg, 92\%). Characterization data was consistent with previously reported data. ${ }^{9} \mathrm{H}$ NMR $\left(400 \mathrm{MHz}, \mathrm{CDCl}_{3}\right) \delta 8.15(\mathrm{~d}, J=15.6 \mathrm{~Hz}, 1 \mathrm{H}), 6.96(\mathrm{~d}, J=15.5$ $\mathrm{Hz}, 1 \mathrm{H}), 4.48(\mathrm{t}, J=8.0 \mathrm{~Hz}, 2 \mathrm{H}), 4.28(\mathrm{q}, J=7.1 \mathrm{~Hz}, 2 \mathrm{H}), 4.11(\mathrm{t}, J=8.0 \mathrm{~Hz}, 2 \mathrm{H}), 1.33(\mathrm{t}, J=7.1 \mathrm{~Hz}$, $3 \mathrm{H})$. 


\section{Rhodium catalyzed coupling reactions}

General procedure for initial experiments evaluating catalytic coupling reactions (Table 1)

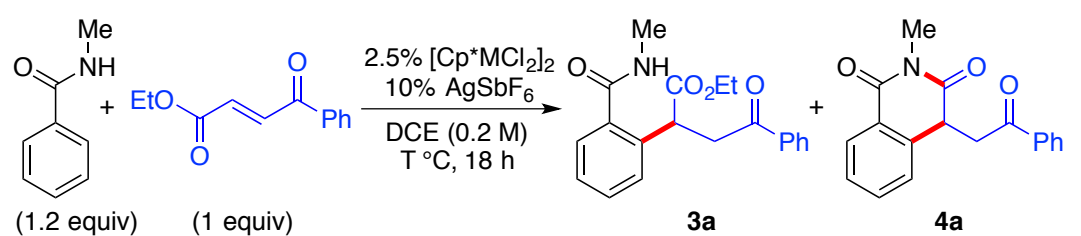

An oven dried Biotage ${ }^{\circledR}$ 0.5-2 mL microwave tube equipped with a stir bar was taken into a nitrogen filled glovebox. $\mathrm{AgSbF}_{6}$ (3.4 mg, $0.010 \mathrm{mmol}, 0.1$ equiv), $N$-methyl benzamide (16 mg, $0.12 \mathrm{mmol}, 1.2$ equiv), and ethyl (E)-4-oxo-4-phenylbut-2-enoate (20 mg, $0.10 \mathrm{mmol}, 1$ equiv) were weighed into the reaction vessel. $\left[\mathrm{Cp}^{*} \mathrm{RhCl}_{2}\right]_{2}$ and $\left[\mathrm{Cp}^{*} \mathrm{CoCl}_{2}\right]_{2}$ were added as freshly prepared solutions in 1,2dichloroethane, such that $\left[\mathrm{Cp}^{*} \mathrm{RhCl}_{2}\right]_{2}(1.6 \mathrm{mg}, 0.0025 \mathrm{mmol}, 0.025$ equiv $)$ or $\left[\mathrm{Cp}^{*} \mathrm{CoCl}_{2}\right]_{2}(1.3 \mathrm{mg}$, $0.0025 \mathrm{mmol}, 0.025$ equiv) could be added in $1.5 \mathrm{~mL}$ of 1,2-dichloroethane. [Cp* $\left.\mathrm{IrCl}_{2}\right]_{2}(2.0 \mathrm{mg}, 0.0025$ mmol, 0.025 equiv) was weighed in directly, and then $1.5 \mathrm{~mL}$ of 1,2-dichloroethane was added. The vessel was sealed using a crimper and the appropriate microwave tube cap with septum. The reaction vessel was removed from the glovebox and submerged in a pre-heated temperature controlled oil bath with stirring. After 18 hours, the reaction vessel was allowed to cool to room temperature, the cap was removed, and the reaction mixture was filtered over celite, eluting with dichloromethane. The reaction mixture was concentrated to an oil and analyzed by ${ }^{1} \mathrm{H}$ NMR spectroscopy with phenyltrimethylsilane added as an external standard.

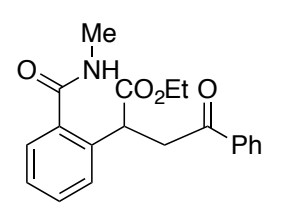

Ethyl 2-(2-(methylcarbamoyl)phenyl)-4-oxo-4-phenylbutanoate 3a: This compound was isolated from the reaction mixture obtained from screening experiment (Table 1, entry 2) with $N$-methyl benzamide (16 mg, $0.12 \mathrm{mmol})$ and ethyl (E)-4-oxo-4phenylbut-2-enoate $(20 \mathrm{mg}, 0.1 \mathrm{mmol})$. The reaction mixture was purified over silica with a $4: 1$ toluene:acetone solvent system to afford the product as a clear oil $(17 \mathrm{mg}, 58 \%$ yield $) .{ }^{1} \mathrm{H}$ $\operatorname{NMR}\left(600 \mathrm{MHz}, \mathrm{CDCl}_{3}\right) \delta 8.00-7.93(\mathrm{~m}, 2 \mathrm{H}), 7.58-7.54(\mathrm{~m}, 1 \mathrm{H}), 7.51(\mathrm{dt}, J=7.3,1.0 \mathrm{~Hz}, 1 \mathrm{H}), 7.45$ $(\mathrm{dd}, J=8.3,7.4 \mathrm{~Hz}, 2 \mathrm{H}), 7.42-7.37$ (m, 2H), 7.32 (ddd, $J=7.6,5.9,2.7 \mathrm{~Hz}, 1 \mathrm{H}), 6.85$ (br s, $1 \mathrm{H}), 4.67$ $(\mathrm{dd}, J=9.2,4.9 \mathrm{~Hz}, 1 \mathrm{H}), 4.19(\mathrm{dq}, J=10.8,7.1 \mathrm{~Hz}, 1 \mathrm{H}), 4.12(\mathrm{dq}, J=10.8,7.1 \mathrm{~Hz}, 1 \mathrm{H}), 3.90(\mathrm{dd}, J=$ $18.2,9.3 \mathrm{~Hz}, 1 \mathrm{H}), 3.53(\mathrm{dd}, J=18.2,4.9 \mathrm{~Hz}, 1 \mathrm{H}), 3.04(\mathrm{~d}, J=4.8 \mathrm{~Hz}, 3 \mathrm{H}), 1.20(\mathrm{t}, J=7.1 \mathrm{~Hz}, 3 \mathrm{H}) ;{ }^{13} \mathrm{C}$ NMR $\left(151 \mathrm{MHz} \mathrm{CDCl}_{3}\right) \delta 197.94,173.28,170.00,137.40,136.48,135.63,133.49,130.39,128.73$, $128.54,128.25,127.79,127.63,61.55,43.09,42.63,26.95,14.17$. IR (neat) $\mathrm{cm}^{-1}: 3311,2980,1729$, 
1684, 1642, 1541, 1174. HRMS (ESI) calculated for $\left[\mathrm{C}_{20} \mathrm{H}_{21} \mathrm{NO}_{4} \mathrm{Na}\right]^{+}$requires $\mathrm{m} / \mathrm{z} 362.1363$, found 362.1364 .

General procedure for catalytic coupling reactions for isolated yields

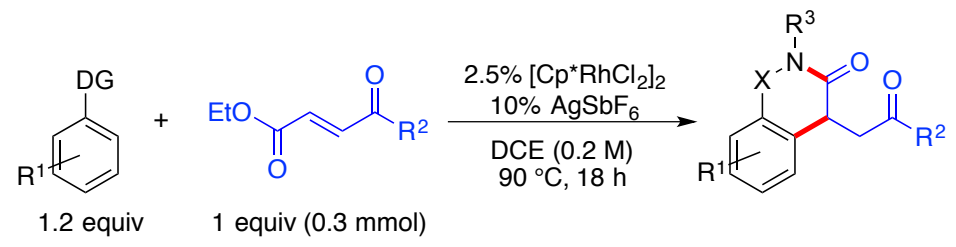

An oven dried Biotage ${ }^{\circledR} 2-5 \mathrm{~mL}$ microwave tube equipped with a stir bar was taken into a nitrogen filled glovebox. [ $\left.\mathrm{Cp}^{*} \mathrm{RhCl}_{2}\right]_{2}$ (4.6 mg, $0.0075 \mathrm{mmol}, 0.025$ equiv), $\mathrm{AgSbF}_{6}(10.3 \mathrm{mg}, 0.030 \mathrm{mmol}, 0.1$ equiv), the indicated $\mathrm{C}-\mathrm{H}$ functionalization substrate $(0.36 \mathrm{mmol}, 1.2$ equiv), and the indicated electrophilic olefin coupling partner $(0.30 \mathrm{mmol}, 1$ equiv $)$ were weighed into the reaction vessel. 1,2-Dichloroethane $(1.5 \mathrm{~mL})$ was added, and the vessel was sealed using a crimper and the appropriate microwave tube cap with septum. The reaction vessel was removed from the glovebox and submerged in a pre-heated temperature controlled oil bath with stirring $\left(90{ }^{\circ} \mathrm{C}\right.$, unless otherwise indicated). After 18 hours, the reaction vessel was allowed to cool to room temperature, the cap was removed and the reaction mixture was filtered over celite, eluting with dichloromethane. The reaction mixture was concentrated to an oil and analyzed by ${ }^{1} \mathrm{H}$ NMR spectroscopy and thin layer chromatography. Purification over silica afforded the pure product.

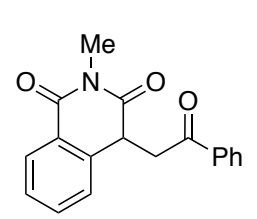

2-Methyl-4-(2-oxo-2-phenylethyl)isoquinoline-1,3(2H,4H)-dione (4a): $\quad$ Prepared according to the general procedure for isolated yields using $\mathrm{C}-\mathrm{H}$ functionalization substrate $N$-methyl benzamide (49 $\mathrm{mg}, 0.36 \mathrm{mmol}$ ) and electrophilic olefin coupling partner ethyl (E)-4-oxo-4-phenylbut-2-enoate $(61 \mathrm{mg}, 0.30 \mathrm{mmol})$. The reaction mixture was purified over silica with a 19:1 toluene:acetone solvent system to afford the product as a white solid $\left(64 \mathrm{mg}, 73 \%\right.$ yield, m.p. $\left.=132-135{ }^{\circ} \mathrm{C}\right) .{ }^{1} \mathrm{H}$ NMR $\left(600 \mathrm{MHz}, \mathrm{CDCl}_{3}\right) \delta 8.28(\mathrm{dd}, J=7.9,1.4$ $\mathrm{Hz}, 1 \mathrm{H}), 7.96-7.88(\mathrm{~m}, 2 \mathrm{H}), 7.60-7.55(\mathrm{~m}, 1 \mathrm{H}), 7.52$ (td, $J=7.6,1.5 \mathrm{~Hz}, 1 \mathrm{H}), 7.48-7.43(\mathrm{~m}, 2 \mathrm{H})$, $7.43-7.39(\mathrm{~m}, 1 \mathrm{H}), 7.27-7.24(\mathrm{~m}, 1 \mathrm{H}), 4.34(\mathrm{t}, J=4.5 \mathrm{~Hz}, 1 \mathrm{H}), 4.07(\mathrm{dd}, J=17.5,4.8 \mathrm{~Hz}, 1 \mathrm{H}), 4.04$ $(\mathrm{dd}, J=17.5,4.2 \mathrm{~Hz}, 1 \mathrm{H}), 3.43$ (s, 3H); ${ }^{13} \mathrm{C} \mathrm{NMR}\left(151 \mathrm{MHz}, \mathrm{CDCl}_{3}\right) \delta 196.31,173.47,165.02,138.24$, $136.14,133.78,133.76,129.38,128.86,128.25,127.69,125.92,125.53,42.22,40.86,27.35$. IR (neat) $\mathrm{cm}^{-1}: 1712,1664,1465,1305,753$. HRMS (ESI) calculated for $\left[\mathrm{C}_{18} \mathrm{H}_{15} \mathrm{NO}_{3} \mathrm{Na}\right]^{+}$requires $\mathrm{m} / z$ 316.0944, found 316.0944 . 


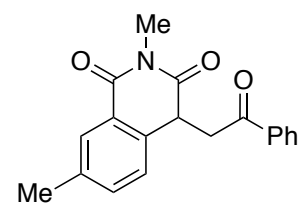

2,7-Dimethyl-4-(2-oxo-2-phenylethyl)isoquinoline-1,3(2H,4H)-dione

(4b):

Prepared according to the general procedure for isolated yields using $\mathrm{C}-\mathrm{H}$ functionalization substrate $N$-methyl 3-methylbenzamide (54 mg, $0.36 \mathrm{mmol}$ ) and electrophilic olefin coupling partner ethyl (E)-4-oxo-4-phenylbut-2-enoate (61 mg,

$0.30 \mathrm{mmol})$. The reaction mixture was purified over silica with a 2:1 hexanes:ethyl acetate solvent system to afford the product as a white solid $\left(82 \mathrm{mg}, 88 \%\right.$ yield, m.p. $\left.=144-148{ }^{\circ} \mathrm{C}\right) .{ }^{1} \mathrm{H}$ NMR $(400 \mathrm{MHz}$, $\left.\mathrm{CDCl}_{3}\right) \delta 8.08(\mathrm{~d}, J=1.9 \mathrm{~Hz}, 1 \mathrm{H}), 7.95-7.88(\mathrm{~m}, 2 \mathrm{H}), 7.61-7.52(\mathrm{~m}, 1 \mathrm{H}), 7.45(\mathrm{dd}, J=8.4,7.1 \mathrm{~Hz}$, 2H), 7.32 (dd, $J=7.9,1.9 \mathrm{~Hz}, 1 \mathrm{H}), 7.14(\mathrm{~d}, J=8.0 \mathrm{~Hz}, 1 \mathrm{H}), 4.29$ (t, $J=4.5 \mathrm{~Hz}, 1 \mathrm{H}), 4.04$ (dd, $J=18.4$, $4.7 \mathrm{~Hz}, 1 \mathrm{H}), 3.99(\mathrm{dd}, J=18.4,4.4 \mathrm{~Hz}, 1 \mathrm{H}), 3.42(\mathrm{~s}, 3 \mathrm{H}), 2.39(\mathrm{~s}, 3 \mathrm{H}) ;{ }^{13} \mathrm{C} \mathrm{NMR}\left(151 \mathrm{MHz}, \mathrm{CDCl}_{3}\right) \delta$ $196.41,173.67,165.20,137.58,136.19,135.30,134.72,133.73,129.51,128.83,128.24,125.63,125.46$, 42.21, 40.60, 27.34, 21.14. IR (neat) $\mathrm{cm}^{-1}: 1703,1680,1654,1302,743$. HRMS (ESI) calculated for $\left[\mathrm{C}_{19} \mathrm{H}_{17} \mathrm{NO}_{3} \mathrm{Na}\right]^{+}$requires $\mathrm{m} / z$ 330.1101, found 330.1100 .

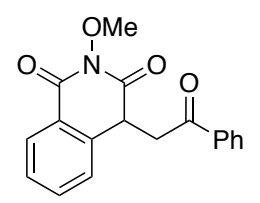

2-Methoxy-4-(2-oxo-2-phenylethyl)isoquinoline-1,3(2H,4H)-dione (4c): Reaction was performed at $60{ }^{\circ} \mathrm{C}$, but otherwise carried out according to the general procedure for isolated yields using $\mathrm{C}-\mathrm{H}$ functionalization substrate $\mathrm{N}$-methoxy benzamide (54 $\mathrm{mg}$, $0.36 \mathrm{mmol}$ ) and electrophilic olefin coupling partner ethyl $(E)$-4-oxo-4-phenylbut-2enoate $(61 \mathrm{mg}, 0.30 \mathrm{mmol})$. The reaction mixture was purified over silica with a 9:1 toluene:acetone solvent system to afford the product as a white solid $\left(86 \mathrm{mg}, 93 \%\right.$ yield, m.p. $\left.=157-159{ }^{\circ} \mathrm{C}\right) .{ }^{1} \mathrm{H} \mathrm{NMR}$ $\left(600 \mathrm{MHz}, \mathrm{CDCl}_{3}\right) \delta 8.29(\mathrm{dd}, J=7.9,1.5 \mathrm{~Hz}, 1 \mathrm{H}), 7.90(\mathrm{dd}, J=8.4,1.3 \mathrm{~Hz}, 2 \mathrm{H}), 7.60-7.56(\mathrm{~m}, 1 \mathrm{H})$, $7.55(\mathrm{td}, J=7.6,1.5 \mathrm{~Hz}, 1 \mathrm{H}), 7.47-7.42(\mathrm{~m}, 3 \mathrm{H}), 7.26(\mathrm{~d}, J=7.7 \mathrm{~Hz}, 1 \mathrm{H}), 4.38(\mathrm{t}, J=4.3 \mathrm{~Hz}, 1 \mathrm{H}), 4.15$ $(\mathrm{dd}, J=18.2,4.9 \mathrm{~Hz}, 1 \mathrm{H}), 4.07(\mathrm{dd}, J=18.3,3.8 \mathrm{~Hz}, 1 \mathrm{H}), 4.05(\mathrm{~s}, 3 \mathrm{H}) ;{ }^{13} \mathrm{C} \mathrm{NMR}\left(151 \mathrm{MHz}, \mathrm{CDCl}_{3}\right) \delta$ 196.00, 169.03, 161.20, 137.52, 135.92, 134.11, 133.92, 129.40, 128.90, 128.24, 127.98, 126.03, 125.79, 63.86, 42.36 (overlapping methine and methylene signals). IR (neat) $\mathrm{cm}^{-1}: 1730,1696,1680,1234,1017$, 752. HRMS (ESI) calculated for $\left[\mathrm{C}_{18} \mathrm{H}_{15} \mathrm{NO}_{4} \mathrm{Na}\right]^{+}$requires $\mathrm{m} / \mathrm{z} 332.0893$, found 332.0895 .

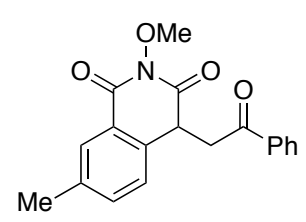

2-Methoxy-7-methyl-4-(2-oxo-2-phenylethyl)isoquinoline-1,3(2H,4H)-dione (4d): Reaction was performed at $60{ }^{\circ} \mathrm{C}$, but otherwise carried out according to the general procedure for isolated yields using $\mathrm{C}-\mathrm{H}$ functionalization substrate $\mathrm{N}$-methoxy 3methylbenzamide ( $60 \mathrm{mg}, 0.36 \mathrm{mmol}$ ) and electrophilic olefin coupling partner ethyl (E)-4-oxo-4-phenylbut-2-enoate $(61 \mathrm{mg}, 0.30 \mathrm{mmol})$. The reaction mixture was purified over silica with a 19:1 toluene:acetone solvent system to afford the product as a white solid (90 mg, 93\% yield, m.p. $=170$ $\left.172{ }^{\circ} \mathrm{C}\right) .{ }^{1} \mathrm{H}$ NMR $\left(400 \mathrm{MHz}, \mathrm{CDCl}_{3}\right) \delta 8.08(\mathrm{~d}, J=1.9 \mathrm{~Hz}, 1 \mathrm{H}), 7.93-7.85(\mathrm{~m}, 2 \mathrm{H}), 7.61-7.53(\mathrm{~m}$, $1 \mathrm{H}), 7.44(\mathrm{t}, J=7.7 \mathrm{~Hz}, 2 \mathrm{H}), 7.34(\mathrm{dd}, J=8.2,1.8 \mathrm{~Hz}, 1 \mathrm{H}), 7.14(\mathrm{~d}, J=7.9 \mathrm{~Hz}, 1 \mathrm{H}), 4.33$ (t, $J=4.3 \mathrm{~Hz}$, 
$1 \mathrm{H}), 4.12(\mathrm{dd}, J=18.2,4.7 \mathrm{~Hz}, 1 \mathrm{H}), 4.04(\mathrm{~s}, 3 \mathrm{H}), 4.03(\mathrm{dd}, J=18.2,4.7 \mathrm{~Hz}, 1 \mathrm{H}), 2.39(\mathrm{~s}, 3 \mathrm{H}) ;{ }^{13} \mathrm{C} \mathrm{NMR}$ $\left(151 \mathrm{MHz}, \mathrm{CDCl}_{3}\right) \delta 196.07,169.20,161.37,138.00,135.98,135.06,134.57,133.87,129.51,128.87$, 128.23, 125.71, 63.82, 42.33, 42.07, 21.08. IR (neat) $\mathrm{cm}^{-1}: 1721,1678,1219,750$. HRMS (ESI) calculated for $\left[\mathrm{C}_{19} \mathrm{H}_{17} \mathrm{NO}_{4} \mathrm{Na}\right]^{+}$requires $m / z 346.1050$, found 346.1053 .

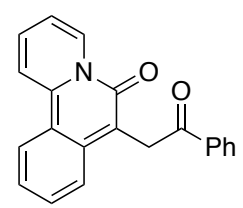

7-(2-Oxo-2-phenylethyl)-6H-pyrido[2,1-a]isoquinolin-6-one (4e): Prepared according to the general procedure for isolated yields using $\mathrm{C}-\mathrm{H}$ functionalization substrate and 1.5 equiv of 2-phenylpyridine (70 $\mathrm{mg}, 0.45 \mathrm{mmol}$ ) and electrophilic olefin coupling partner ethyl $(E)$-4-oxo-4-phenylbut-2-enoate $(61 \mathrm{mg}, 0.30 \mathrm{mmol})$. The reaction mixture was purified over silica with a 4:1 dichloromethane:acetone solvent system to afford the product as an orange solid $\left(74 \mathrm{mg}, 79 \%\right.$ yield, m.p. $\left.=182-189{ }^{\circ} \mathrm{C}\right) .{ }^{1} \mathrm{H}$ NMR $\left(600 \mathrm{MHz}, \mathrm{CDCl}_{3}\right) \delta 9.93(\mathrm{~d}, J=6.9 \mathrm{~Hz}, 1 \mathrm{H})$, $8.71(\mathrm{~d}, J=8.8 \mathrm{~Hz}, 1 \mathrm{H}), 8.36(\mathrm{~d}, J=8.1 \mathrm{~Hz}, 1 \mathrm{H}), 8.28-8.25(\mathrm{~m}, 2 \mathrm{H}), 7.93(\mathrm{ddd}, J=8.6,6.9,1.4 \mathrm{~Hz}$, $1 \mathrm{H}), 7.71(\mathrm{~d}, J=8.6 \mathrm{~Hz}, 1 \mathrm{H}), 7.62-7.52(\mathrm{~m}, 3 \mathrm{H}), 7.46(\mathrm{t}, J=7.7 \mathrm{~Hz}, 2 \mathrm{H}), 7.23(\mathrm{ddd}, J=8.3,6.7,1.1$ $\mathrm{Hz}, 1 \mathrm{H}), 4.88(\mathrm{~s}, 2 \mathrm{H}) ;{ }^{13} \mathrm{C} \mathrm{NMR}\left(151 \mathrm{MHz}, \mathrm{CDCl}_{3}\right) \delta 199.09,154.06,141.73,138.31,137.16,133.10$, 132.97, 131.97, 130.79, 128.78, 128.63, 124.20, 122.52, 121.55, 121.02, 119.04, 114.38, 102.24, 38.26. IR (neat) $\mathrm{cm}^{-1}: 1668,1611,1278,769$. HRMS (ESI) calculated for $\left[\mathrm{C}_{21} \mathrm{H}_{15} \mathrm{NO}_{2} \mathrm{Na}\right]^{+}$requires $\mathrm{m} / z$ 336.0995, found 336.0993.

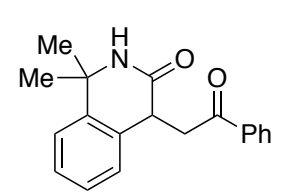

1,1-Dimethyl-4-(2-oxo-2-phenylethyl)-1,4-dihydroisoquinolin-3(2H)-one

(4f): Prepared according to the general procedure for isolated yields using $\mathrm{C}-\mathrm{H}$ functionalization substrate cumylamine $(49 \mathrm{mg}, 0.36 \mathrm{mmol})$ and electrophilic olefin coupling partner ethyl (E)-4-oxo-4-phenylbut-2-enoate $(61 \mathrm{mg}, 0.30 \mathrm{mmol})$. The reaction mixture was purified over silica with a 3:2 hexanes:acetone solvent system to afford the product as a white solid $\left(50 \mathrm{mg}, 57 \%\right.$ yield, m.p. $\left.=195-198{ }^{\circ} \mathrm{C}\right) .{ }^{1} \mathrm{H}$ NMR $\left(600 \mathrm{MHz}, \mathrm{CDCl}_{3}\right) \delta 8.05-8.02(\mathrm{~m}$, 2H), $7.60-7.56(\mathrm{~m}, 1 \mathrm{H}), 7.48(\mathrm{t}, J=7.8 \mathrm{~Hz}, 2 \mathrm{H}), 7.31(\mathrm{dd}, J=7.9,1.4 \mathrm{~Hz}, 1 \mathrm{H}), 7.25(\mathrm{t}, J=7.5 \mathrm{~Hz}, 1 \mathrm{H})$, $7.19(\mathrm{td}, J=7.5,1.4 \mathrm{~Hz}, 1 \mathrm{H}), 7.07(\mathrm{~d}, J=7.8 \mathrm{~Hz}, 1 \mathrm{H}), 6.22(\mathrm{~s}, 1 \mathrm{H}), 4.36(\mathrm{dd}, J=6.8,4.1 \mathrm{~Hz}, 1 \mathrm{H}), 4.06$ $(\mathrm{dd}, J=17.7,4.1 \mathrm{~Hz}, 1 \mathrm{H}), 3.66(\mathrm{dd}, J=17.7,6.7 \mathrm{~Hz}, 1 \mathrm{H}), 1.72(\mathrm{~s}, 3 \mathrm{H}), 1.59(\mathrm{~s}, 3 \mathrm{H}) ;{ }^{13} \mathrm{C}$ NMR $(151$ $\left.\mathrm{MHz}, \mathrm{CDCl}_{3}\right) \delta 197.73,171.90,139.96,136.88,133.88,133.34,128.76,128.37,127.66,127.11,126.41$, 124.01, 55.97, 40.35, 39.66, 32.19, 31.54. IR (neat) $\mathrm{cm}^{-1}: 3199,1678,1653,1448,1238,755$. HRMS (ESI) calculated for $\left[\mathrm{C}_{19} \mathrm{H}_{19} \mathrm{NO}_{2} \mathrm{Na}\right]^{+}$requires $\mathrm{m} / z$ 316.1308, found 316.1304 .

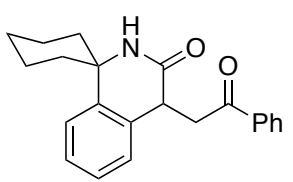

4'-(2-Oxo-2-phenylethyl)-2' $H$-spiro[cyclohexane-1,1'-isoquinolin]-3'(4'H)-one $\quad(\mathbf{4 g})$ : Prepared according to the general procedure for isolated yields using $\mathrm{C}-\mathrm{H}$ functionalization substrate 1-phenylcyclohexan-1-amine (63 $\mathrm{mg}, 0.36 \mathrm{mmol})$ and 
electrophilic olefin coupling partner ethyl (E)-4-oxo-4-phenylbut-2-enoate $(61 \mathrm{mg}, 0.30 \mathrm{mmol})$. The reaction mixture was purified over silica with a 7:3 hexanes:acetone solvent system to afford the product as a white solid $\left(67 \mathrm{mg}, 67 \%\right.$ yield, m.p. $\left.=167-168{ }^{\circ} \mathrm{C}\right) .{ }^{1} \mathrm{H}$ NMR $\left(600 \mathrm{MHz}, \mathrm{CDCl}_{3}\right) \delta 8.07-8.02(\mathrm{~m}$, 2H), $7.62-7.55(\mathrm{~m}, 1 \mathrm{H}), 7.51-7.46(\mathrm{~m}, 2 \mathrm{H}), 7.36(\mathrm{dd}, J=7.9,1.5 \mathrm{~Hz}, 1 \mathrm{H}), 7.26(\mathrm{tt}, J=7.7,1.1 \mathrm{~Hz}$, $1 \mathrm{H}), 7.19(\mathrm{td}, J=7.5,1.3 \mathrm{~Hz}, 1 \mathrm{H}), 7.05(\mathrm{~d}, J=7.8 \mathrm{~Hz}, 1 \mathrm{H}), 6.47(\mathrm{~s}, 1 \mathrm{H}), 4.39$ (dd, $J=6.9,4.0 \mathrm{~Hz}, 1 \mathrm{H})$, $4.07(\mathrm{dd}, J=17.8,4.0 \mathrm{~Hz}, 1 \mathrm{H}), 3.63(\mathrm{dd}, J=17.8,6.9 \mathrm{~Hz}, 1 \mathrm{H}), 2.13(\mathrm{td}, J=13.6,4.3 \mathrm{~Hz}, 1 \mathrm{H}), 2.04-$ $1.94(\mathrm{~m}, 2 \mathrm{H}), 1.91-1.81(\mathrm{~m}, 2 \mathrm{H}), 1.75(\mathrm{dt}, J=16.1,2.6 \mathrm{~Hz}, 1 \mathrm{H}), 1.72-1.55(\mathrm{~m}, 3 \mathrm{H}), 1.35$ (qt, $J=13.0$, $3.7 \mathrm{~Hz}, 1 \mathrm{H}) ;{ }^{13} \mathrm{C}$ NMR $\left(151 \mathrm{MHz}, \mathrm{CDCl}_{3}\right) \delta 197.80,171.86,140.42,136.89,134.50,133.34,128.76$, $128.39,127.63,127.06,126.32,123.83,57.30,40.10,39.61,38.65,37.49,25.12,21.92,21.76$. IR (neat) $\mathrm{cm}^{-1}: 3201,3061,2926,1686,1651,1425,1255,749$. HRMS (ESI) calculated for $\left[\mathrm{C}_{22} \mathrm{H}_{23} \mathrm{NO}_{2} \mathrm{Na}\right]^{+}$ requires $m / z 356.1621$, found 356.1619 .

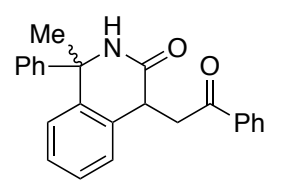

1-Methyl-4-(2-oxo-2-phenylethyl)-1-phenyl-1,4-dihydroisoquinolin-3(2H)-one

(4h): Prepared according to the general procedure for isolated yields using $\mathrm{C}-\mathrm{H}$ functionalization substrate 1,1-diphenylethan-1-amine $(71 \mathrm{mg}, 0.36 \mathrm{mmol})$ and electrophilic olefin coupling partner ethyl (E)-4-oxo-4-phenylbut-2-enoate (61 mg, $0.30 \mathrm{mmol}) .{ }^{1} \mathrm{H}$ NMR Spectroscopic analysis of the crude reaction mixture revealed a 2.7:1 mixture of diastereomeric products. The reaction mixture was purified over silica with a 3:2 hexanes:ethyl acetate solvent system. The minor diastereomer eluted first, affording a white solid $\left(22 \mathrm{mg}, 21 \%\right.$ yield, m.p. $\left.=170-173{ }^{\circ} \mathrm{C}\right)$, and the major diastereomer eluted second, affording a white solid (65 mg, 61\% yield, m.p. $\left.=205-209{ }^{\circ} \mathrm{C}\right)$. The relative stereochemistry was characterized based on NOESY-1D ${ }^{1} \mathrm{H}$ NMR spectroscopic analysis of the purified diastereomers. For the major diastereomer, irradiation of the signal corresponding to the methyl protons $(1.96 \mathrm{ppm})$ resulted in an NOE signal corresponding to the methine proton (4.42 ppm), and vice versa. For the minor diastereomer, the corresponding NOE signal between the methyl protons $(1.97 \mathrm{ppm})$ and methine proton (overlapping with a methylene peak, $4.05-3.96 \mathrm{ppm}$ ) was not observed.

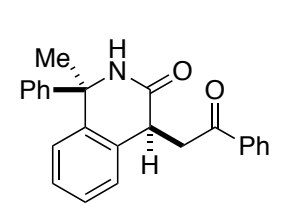

Major diastereomer: ${ }^{1} \mathrm{H}$ NMR $\left(600 \mathrm{MHz}, \mathrm{CDCl}_{3}\right) \delta 7.80(\mathrm{dd}, J=8.3,1.4 \mathrm{~Hz}, 2 \mathrm{H})$, $7.56-7.51(\mathrm{~m}, 1 \mathrm{H}), 7.50-7.46(\mathrm{~m}, 2 \mathrm{H}), 7.45-7.28(\mathrm{~m}, 6 \mathrm{H}), 7.22-7.17(\mathrm{~m}, 3 \mathrm{H})$, $7.07-7.02(\mathrm{~m}, 1 \mathrm{H}), 6.40(\mathrm{~s}, 1 \mathrm{H}), 4.42(\mathrm{dd}, J=7.7,3.7 \mathrm{~Hz}, 1 \mathrm{H}), 3.70(\mathrm{dd}, J=17.6$, $3.8 \mathrm{~Hz}, 1 \mathrm{H}), 3.34(\mathrm{dd}, J=17.6,7.8 \mathrm{~Hz}, 1 \mathrm{H}), 1.96(\mathrm{~s}, 3 \mathrm{H}) ;{ }^{13} \mathrm{C}$ NMR $(151 \mathrm{MHz}$, $\left.\mathrm{CDCl}_{3}\right) \delta 197.25,172.58,146.67,139.80,136.76,134.61,133.32,128.85,128.68,128.29,127.88$, $127.87,127.40,126.94,126.77,126.27,61.53,40.66,40.12,30.67$. IR (neat) $\mathrm{cm}^{-1}: 3179,3055,2893$, $1684,1660,1399,1247,747$. HRMS (ESI) calculated for $\left[\mathrm{C}_{24} \mathrm{H}_{21} \mathrm{NO}_{2} \mathrm{Na}\right]^{+}$requires $m / z 378.1465$, found 378.1462 . 


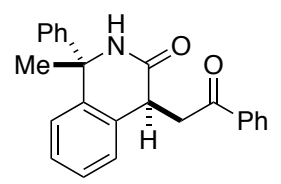

Minor diastereomer: ${ }^{1} \mathrm{H}$ NMR $\left(600 \mathrm{MHz}, \mathrm{CDCl}_{3}\right) \delta 8.07-8.02(\mathrm{~m}, 2 \mathrm{H}), 7.61-7.55$ $(\mathrm{m}, 1 \mathrm{H}), 7.51-7.45(\mathrm{~m}, 3 \mathrm{H}), 7.36-7.22(\mathrm{~m}, 7 \mathrm{H}), 7.04-7.00(\mathrm{~m}, 1 \mathrm{H}), 6.60(\mathrm{~s}, 1 \mathrm{H})$, $4.05-3.96(\mathrm{~m}, 2 \mathrm{H}), 3.57(\mathrm{dd}, J=16.8,5.7 \mathrm{~Hz}, 1 \mathrm{H}), 1.97(\mathrm{~s}, 3 \mathrm{H}) ;{ }^{13} \mathrm{C}$ NMR $(151$ $\left.\mathrm{MHz} \mathrm{CDCl}_{3}\right) \delta 197.65,173.31,144.98,140.25,136.92,135.11,133.31,128.76,128.73,128.37,127.94$, $127.51,126.77,125.69,125.35,124.88,60.89,40.97,37.09,30.66$. IR (neat) $\mathrm{cm}^{-1}: 3232,3065,2911$, $1676,1645,1447,1203,747$. HRMS (ESI) calculated for $\left[\mathrm{C}_{24} \mathrm{H}_{21} \mathrm{NO}_{2} \mathrm{Na}\right]^{+}$requires $\mathrm{m} / z 378.1465$, found 378.1460 .

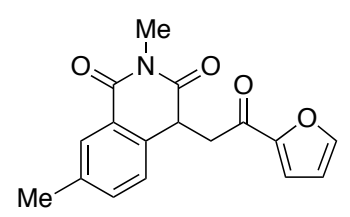

4-(2-(Furan-2-yl)-2-oxoethyl)-2,7-dimethylisoquinoline-1,3(2H,4H)-dione (4i): Prepared according to the general procedure for isolated yields using $\mathrm{C}-\mathrm{H}$ functionalization substrate $N$-methyl 3-methylbenzamide (54 $\mathrm{mg}, 0.36 \mathrm{mmol}$ ) and electrophilic olefin coupling partner ethyl (E)-4-(2-furyl)-4-oxo-but-2-enoate (58 $\mathrm{mg}, 0.30 \mathrm{mmol})$. The reaction mixture was purified over silica with a 9:1 toluene:acetone solvent system to afford the product as a tan solid $\left(78 \mathrm{mg}, 87 \%\right.$ yield, m.p. $\left.=144-146{ }^{\circ} \mathrm{C}\right) .{ }^{1} \mathrm{H} \mathrm{NMR}\left(600 \mathrm{MHz}, \mathrm{CDCl}_{3}\right) \delta$ $8.07-8.05(\mathrm{~m} \mathrm{1H}), 7.59-7.55(\mathrm{~m}, 1 \mathrm{H}), 7.34(\mathrm{dd}, J=7.9,1.9 \mathrm{~Hz}, 1 \mathrm{H}), 7.19-7.15(\mathrm{~m}, 2 \mathrm{H}), 6.53(\mathrm{dd}, J=$ 3.6, $1.7 \mathrm{~Hz}, 1 \mathrm{H}), 4.29$ (t, $J=4.8 \mathrm{~Hz}, 1 \mathrm{H}), 3.87$ (dd, $J=18.0,4.4 \mathrm{~Hz}, 1 \mathrm{H}), 3.82$ (dd, $J=18.0,5.2 \mathrm{~Hz}, 1 \mathrm{H})$, $3.40(\mathrm{~s}, 3 \mathrm{H}), 2.39$ (s, 3H); ${ }^{13} \mathrm{C}$ NMR (151 MHz, $\left.\mathrm{CDCl}_{3}\right) \delta 185.68,173.49,165.10,152.29,146.72,137.70$, 135.04, 134.74, 129.51, 125.71, 125.55, 117.53, 112.64, 42.15, 40.38, 27.34, 21.15. IR (neat) $\mathrm{cm}^{-1}: 1699$, 1649, 1400, 1307, 779. HRMS (ESI) calculated for $\left[\mathrm{C}_{17} \mathrm{H}_{15} \mathrm{NO}_{4} \mathrm{Na}\right]^{+}$requires $\mathrm{m} / z 320.0893$, found 320.0891 .

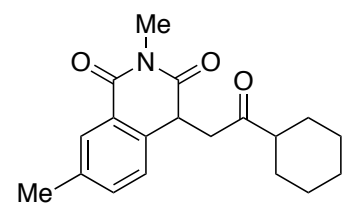

4-(2-Cyclohexyl-2-oxoethyl)-2,7-dimethylisoquinoline-1,3(2H,4H)-dione $\quad(\mathbf{4 j})$ : Prepared according to the general procedure for isolated yields using $\mathrm{C}-\mathrm{H}$ functionalization substrate $N$-methyl 3-methylbenzamide (54 $\mathrm{mg}, 0.36 \mathrm{mmol}$ ) and electrophilic olefin coupling partner ethyl (E)-4-cyclohexyl-4-oxobut-2-enoate $(63 \mathrm{mg}, 0.30 \mathrm{mmol})$. The reaction mixture was purified over silica with a 3:1 hexanes:acetone solvent system to afford the product as a pale yellow solid $\left(73 \mathrm{mg}, 78 \%\right.$ yield, m.p. $\left.=77-82{ }^{\circ} \mathrm{C}\right) .{ }^{1} \mathrm{H}$ NMR $(600$ $\left.\mathrm{MHz}, \mathrm{CDCl}_{3}\right) \delta 8.03(\mathrm{~s}, 1 \mathrm{H}), 7.34(\mathrm{~d}, J=7.8 \mathrm{~Hz}, 1 \mathrm{H}), 7.07(\mathrm{~d}, J=7.9 \mathrm{~Hz}, 1 \mathrm{H}), 4.10(\mathrm{t}, J=4.7 \mathrm{~Hz}, 1 \mathrm{H})$, $3.45(\mathrm{dd}, J=18.4,4.9 \mathrm{~Hz}, 1 \mathrm{H}), 3.41(\mathrm{dd}, J=18.4,4.6 \mathrm{~Hz}, 1 \mathrm{H}), 3.37(\mathrm{~s}, 3 \mathrm{H}), 2.39(\mathrm{~s}, 3 \mathrm{H}), 2.30(\mathrm{tt}, J=$ 10.8, $3.3 \mathrm{~Hz}, 1 \mathrm{H}), 1.82-1.70(\mathrm{~m}, 4 \mathrm{H}), 1.66-1.60(\mathrm{~m}, 1 \mathrm{H}), 1.32-1.09(\mathrm{~m}, 5 \mathrm{H}) ;{ }^{13} \mathrm{C}$ NMR $(151 \mathrm{MHz}$, $\left.\mathrm{CDCl}_{3}\right) \delta 210.30,173.70,165.09,137.50,135.39,134.62,129.45,125.49,125.37,50.58,44.12,40.39$, 28.34, 28.31, 27.27, 25.85, 25.65, 25.62, 21.12. IR (neat) $\mathrm{cm}^{-1}: 2927,2852,1705,1695,1660,1440$, 1302, 1144, 821. HRMS (ESI) calculated for $\left[\mathrm{C}_{19} \mathrm{H}_{23} \mathrm{NO}_{3} \mathrm{Na}\right]^{+}$requires $\mathrm{m} / z$ 336.1570, found 336.1568. 


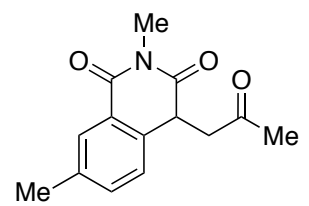

2,7-Dimethyl-4-(2-oxopropyl)isoquinoline-1,3(2H,4H)-dione (4k): $\quad$ Prepared according to the general procedure for isolated yields using $\mathrm{C}-\mathrm{H}$ functionalization substrate $N$-methyl 3-methylbenzamide (54 mg, $0.36 \mathrm{mmol}$ ) and electrophilic olefin coupling partner ethyl (E)-4-oxopent-2-enoate (43 $\mathrm{mg}, 0.30 \mathrm{mmol})$. The reaction mixture was purified over silica with a 9:1 toluene:acetone solvent system to afford the product as a white solid $\left(62 \mathrm{mg}, 84 \%\right.$ yield, m.p. $\left.=107-109{ }^{\circ} \mathrm{C}\right) .{ }^{1} \mathrm{H}$ NMR $\left(600 \mathrm{MHz}, \mathrm{CDCl}_{3}\right) \delta 8.06-8.04(\mathrm{~m}, 1 \mathrm{H}), 7.36$ $(\mathrm{dd}, J=8.0,1.9 \mathrm{~Hz}, 1 \mathrm{H}), 7.11(\mathrm{~d}, J=7.9 \mathrm{~Hz}, 1 \mathrm{H}), 4.11(\mathrm{t}, J=4.7 \mathrm{~Hz}, 1 \mathrm{H}), 3.44(\mathrm{dd}, J=18.2,5.1 \mathrm{~Hz}$, $1 \mathrm{H}), 3.38(\mathrm{dd}, J=18.3,4.3 \mathrm{~Hz}, 1 \mathrm{H}), 3.38(\mathrm{~s}, 3 \mathrm{H}), 2.41$ (s, 3H), $2.14(\mathrm{~s}, 3 \mathrm{H}) ;{ }^{13} \mathrm{C} \mathrm{NMR}\left(151 \mathrm{MHz}, \mathrm{CDCl}_{3}\right)$ $\delta 204.96,173.55,165.06,137.68,135.14,134.74,129.49,125.50,46.94,40.54,30.04,27.28,21.14$. IR (neat) $\mathrm{cm}^{-1}: 1700,1651,1615,1304,1139,782$. HRMS (ESI) calculated for $\left[\mathrm{C}_{14} \mathrm{H}_{15} \mathrm{NO}_{3} \mathrm{Na}\right]^{+}$requires $m / z$ 268.0944 , found 268.0949 .

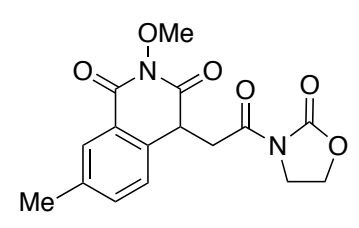

2-Methoxy-7-methyl-4-(2-oxo-2-(2-oxooxazolidin-3-yl)ethyl)isoquinoline1,3(2H,4H)-dione (41): Reaction was performed at $60{ }^{\circ} \mathrm{C}$, but otherwise carried out according to the general procedure for isolated yields using $\mathrm{C}-\mathrm{H}$ functionalization substrate $N$-methoxy 3-methylbenzamide (60 $\mathrm{mg}, 0.36 \mathrm{mmol}$ ) and electrophilic olefin coupling partner ethyl (E)-4-oxo-4-(2-oxooxazolidin-3-yl)but-2-enoate (64 mg, $0.30 \mathrm{mmol}$ ). The reaction mixture was purified over silica with a 3:2 hexanes:acetone solvent system to afford the product as a white solid $\left(62 \mathrm{mg}, 84 \%\right.$ yield, m.p. $\left.=157-162{ }^{\circ} \mathrm{C}\right) .{ }^{1} \mathrm{H} \mathrm{NMR}\left(600 \mathrm{MHz}, \mathrm{CDCl}_{3}\right) \delta$ $8.09-8.05(\mathrm{~m}, 1 \mathrm{H}), 7.41(\mathrm{dd}, J=7.9,1.9 \mathrm{~Hz}, 1 \mathrm{H}), 7.21(\mathrm{~d}, J=7.6 \mathrm{~Hz}, 1 \mathrm{H}), 4.44-4.37(\mathrm{~m}, 1 \mathrm{H}), 4.40-$ $4.33(\mathrm{~m}, 1 \mathrm{H}), 4.22(\mathrm{t}, J=4.5 \mathrm{~Hz}, 1 \mathrm{H}), 4.04(\mathrm{dd}, J=18.4,4.5 \mathrm{~Hz}, 1 \mathrm{H}), 4.00(\mathrm{~s}, 3 \mathrm{H}), 3.99-3.88(\mathrm{~m}, 2 \mathrm{H})$, $3.84(\mathrm{ddd}, J=11.0,9.2,7.2 \mathrm{~Hz}, 1 \mathrm{H}), 2.42(\mathrm{~s}, 3 \mathrm{H}) ;{ }^{13} \mathrm{C} \mathrm{NMR}\left(151 \mathrm{MHz}, \mathrm{CDCl}_{3}\right) \delta 169.91,168.77,161.23$, $153.69,138.25,135.18,133.98,129.50,125.92,125.73,63.81,62.53,42.35,42.21,39.23$, 21.13. IR (neat) $\mathrm{cm}^{-1}: 1775,1722,1678,1396,1207,1023,757$. HRMS (ESI) calculated for $\left[\mathrm{C}_{16} \mathrm{H}_{16} \mathrm{~N}_{2} \mathrm{O}_{6} \mathrm{Na}\right]^{+}$ requires $m / z$ 355.0901, found 355.0902 .

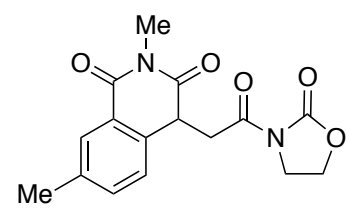

2,7-Dimethyl-4-(2-oxo-2-(2-oxooxazolidin-3-yl)ethyl)isoquinoline-1,3(2H,4H)dione (4m): Prepared according to the general procedure for isolated yields using $\mathrm{C}-\mathrm{H}$ functionalization substrate $\mathrm{N}$-methyl 3-methylbenzamide $(54 \mathrm{mg}, 0.36$ mmol) and electrophilic olefin coupling partner ethyl (E)-4-oxo-4-(2oxooxazolidin-3-yl)but-2-enoate $(64 \mathrm{mg}, 0.30 \mathrm{mmol})$. The reaction mixture was purified over silica with a 85:15 toluene:acetone solvent system to afford the product as a white solid $(63 \mathrm{mg}, 66 \%$ yield, m.p. $=$ 196-199 $\left.{ }^{\circ} \mathrm{C}\right) .{ }^{1} \mathrm{H}$ NMR $\left(600 \mathrm{MHz}, \mathrm{CDCl}_{3}\right) \delta 8.06(\mathrm{~s}, 1 \mathrm{H}), 7.39(\mathrm{~d}, J=7.9 \mathrm{~Hz}, 1 \mathrm{H}), 7.22(\mathrm{~d}, J=7.9 \mathrm{~Hz}$, 1H), $4.43-4.34(\mathrm{~m}, 2 \mathrm{H}), 4.14(\mathrm{t}, J=4.8 \mathrm{~Hz}, 1 \mathrm{H}), 3.97(\mathrm{dd}, J=18.2,4.9 \mathrm{~Hz}, 1 \mathrm{H}), 3.94-3.83(\mathrm{~m}, 3 \mathrm{H})$, 
$3.38(\mathrm{~s}, 3 \mathrm{H}), 2.41$ (s, 3H); ${ }^{13} \mathrm{C}$ NMR (151 MHz, $\left.\mathrm{CDCl}_{3}\right) \delta 173.19,170.20,165.00,153.72,137.80,134.80$, $134.65,129.50,125.65,125.57,62.48,42.39,40.77,39.08,27.25,21.17$. IR (neat) $\mathrm{cm}^{-1}: 1775,1695$, $1658,1392,1207,1118,1035,758$. HRMS (ESI) calculated for $\left[\mathrm{C}_{16} \mathrm{H}_{16} \mathrm{~N}_{2} \mathrm{O}_{5} \mathrm{Na}\right]^{+}$requires $\mathrm{m} / z 339.0951$, found 339.0949 . 


\section{Synthesis of oxoassoanine}
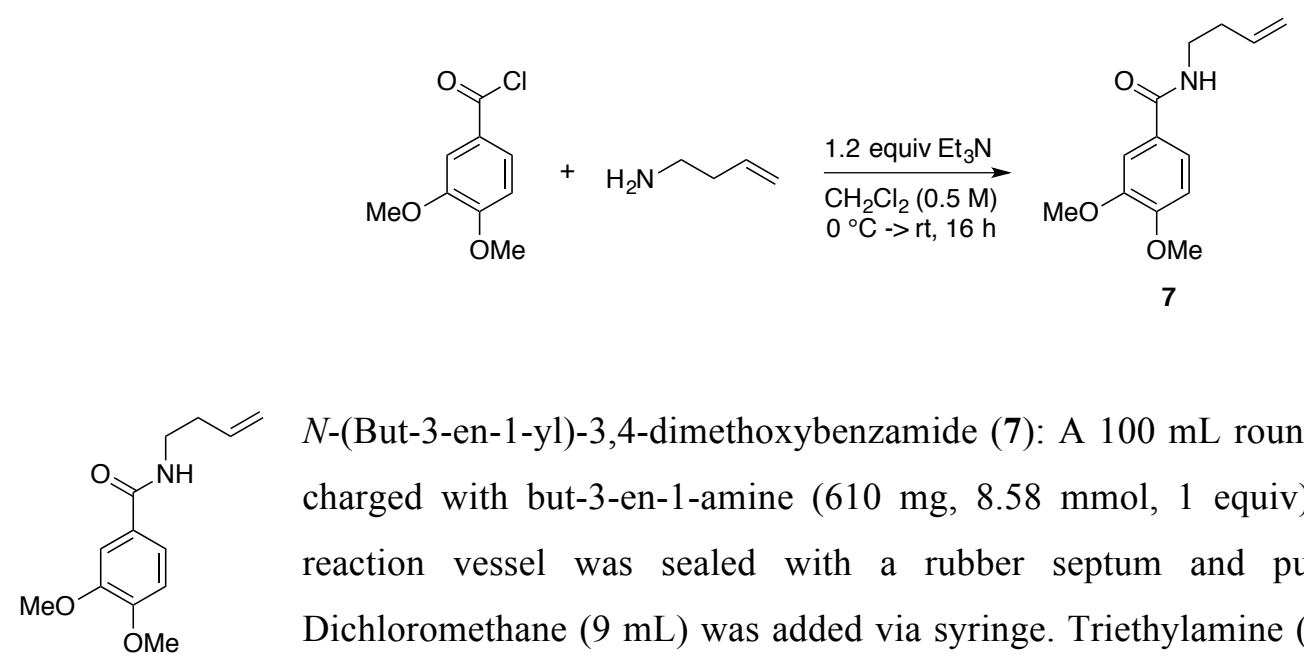

$N$-(But-3-en-1-yl)-3,4-dimethoxybenzamide (7): A $100 \mathrm{~mL}$ round bottomed flask was charged with but-3-en-1-amine $(610 \mathrm{mg}, 8.58 \mathrm{mmol}, 1$ equiv) and a stir bar. The reaction vessel was sealed with a rubber septum and purged with nitrogen. Dichloromethane $(9 \mathrm{~mL})$ was added via syringe. Triethylamine $(1.43 \mathrm{~mL}, 10.3 \mathrm{mmol}$, 1.2 equiv) was added via syringe, and the reaction mixture was cooled to $0{ }^{\circ} \mathrm{C}$. 3,4-Dimethoxybenzoyl chloride was weighed into a separate $50 \mathrm{~mL}$ pear shaped flask, flushed with nitrogen and dissolved in 9 $\mathrm{mL}$ of dichloromethane. The solution of benzoyl chloride was added dropwise to the reaction vessel at 0 ${ }^{\circ} \mathrm{C}$ via cannula. The reaction mixture was stirred at $0{ }^{\circ} \mathrm{C}$ and slowly warmed to room temperature overnight $(16 \mathrm{~h})$. The reaction mixture was partitioned between $100 \mathrm{~mL}$ of diethyl ether and $100 \mathrm{~mL} 1 \mathrm{M}$ $\mathrm{HCl}$ in a separatory funnel. The aqueous layer was re-extracted $2 \mathrm{x}$ with ether. The combined organics were washed with saturated sodium bicarbonate and brine, dried over magnesium sulfate, and concentrated to an oil. The residue was purified over silica using a 1:1 hexanes:ethyl acetate solvent system to afford the product as a white solid $\left(1.475 \mathrm{~g}, 73 \%\right.$, m.p. $\left.=96-99{ }^{\circ} \mathrm{C}\right) .{ }^{1} \mathrm{H} \mathrm{NMR}(600 \mathrm{MHz}$, $\left.\mathrm{CDCl}_{3}\right) \delta 7.41(\mathrm{~d}, J=2.0 \mathrm{~Hz}, 1 \mathrm{H}), 7.22(\mathrm{dd}, J=8.3,2.1 \mathrm{~Hz}, 1 \mathrm{H}), 6.85(\mathrm{~d}, J=8.3 \mathrm{~Hz}, 1 \mathrm{H}), 6.13(\mathrm{~s}, 1 \mathrm{H})$, $5.83(\mathrm{ddt}, J=17.1,10.1,6.9 \mathrm{~Hz}, 1 \mathrm{H}), 5.20-5.09(\mathrm{~m}, 2 \mathrm{H}), 3.92(\mathrm{~s}, 3 \mathrm{H}), 3.91$ (s, 3H), 3.52 (q, $J=6.4 \mathrm{~Hz}$, 2H), $2.38(\mathrm{q}, J=6.8 \mathrm{~Hz}, 2 \mathrm{H}) ;{ }^{13} \mathrm{C}$ NMR $\left(151 \mathrm{MHz}, \mathrm{CDCl}_{3}\right) \delta 167.13,151.76,149.12,135.58,127.55$, 119.12, 117.52, 110.73, 110.35, 56.14, 38.93, 33.99. IR (neat) $\mathrm{cm}^{-1}: 3286,1629,1542,1511,1231,1134$, 1022, 923. HRMS (ESI) calculated for $\left[\mathrm{C}_{13} \mathrm{H}_{17} \mathrm{NO}_{3} \mathrm{Na}\right]^{+}$requires $m / z 258.1101$, found 258.1101 .

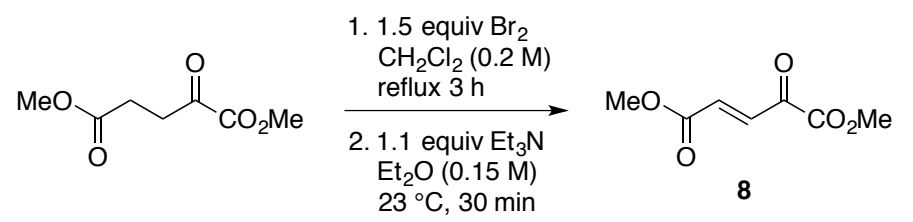

Dimethyl $(E)$-4-oxopent-2-enedioate (8): Based on a literature procedure, ${ }^{10}$ an oven
dried $250 \mathrm{~mL}$ three-necked round bottomed flask equipped with a stir bar and reflux condenser was purged with nitrogen. Dimethyl 2-oxoglutarate $(1.45 \mathrm{~mL}, 10.0 \mathrm{mmol}, 1$ equiv) and 
dichloromethane $(50 \mathrm{~mL})$ were added via syringe, and the mixture was heated to reflux. A $25 \mathrm{~mL}$ pear shaped flask was purged with nitrogen and charged with bromine $(0.77 \mathrm{~mL}, 15 \mathrm{mmol}, 1.5$ equiv) and 2 $\mathrm{mL}$ of dichloromethane. The bromine solution was added slowly to the reaction mixture via syringe, and the mixture was heated to reflux for 3 hours. The reaction mixture was cooled to room temperature and concentrated under a stream of nitrogen set to vent through a bubbler filled with saturated sodium bicarbonate solution. The red orange oil was suspended in diethyl ether $(67 \mathrm{~mL})$, and triethylamine $(1.52$ $\mathrm{mL}, 11.0 \mathrm{mmol}, 1.1$ equiv) was added via syringe. The reaction mixture was stirred at room temperature for 30 minutes, with solids precipitating. The reaction mixture was passed through a plug of silica, eluting diethyl ether, and concentrated to a yellow solid. The crude product was purified over silica using a 2:1 pentane:diethyl ether solvent system to afford the product as a yellow solid $(1.13 \mathrm{~g}, 70 \%$ yield, m.p. $=65$ $\left.70{ }^{\circ} \mathrm{C}\right)$. The characterization data was consistent with previously reported data. ${ }^{10}{ }^{1} \mathrm{H} \mathrm{NMR}(600 \mathrm{MHz}$, $\left.\mathrm{CDCl}_{3}\right) \delta 7.63(\mathrm{~d}, J=16.0 \mathrm{~Hz}, 1 \mathrm{H}), 6.98(\mathrm{~d}, J=16.0 \mathrm{~Hz}, 1 \mathrm{H}), 3.94(\mathrm{~s}, 3 \mathrm{H}), 3.84(\mathrm{~s}, 3 \mathrm{H}) ;{ }^{13} \mathrm{C}$ NMR $(151$ $\left.\mathrm{MHz}, \mathrm{CDCl}_{3}\right) \delta 182.29,165.24,161.10,135.61,134.22,53.54,52.77$.

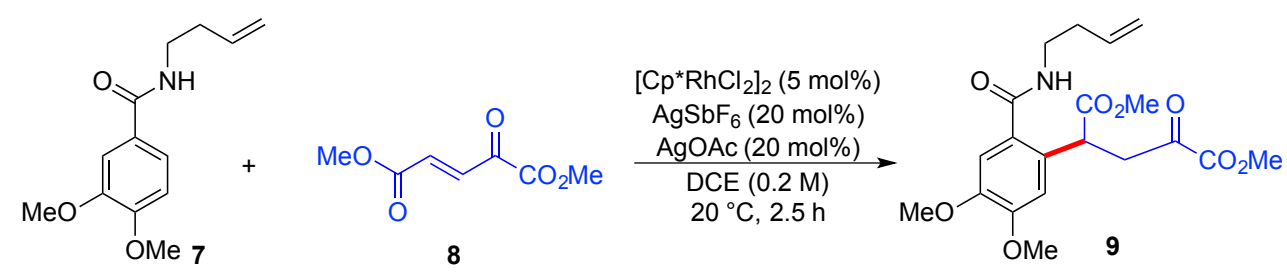

Dimethyl 2-(2-(but-3-en-1-ylcarbamoyl)-4,5-dimethoxyphenyl)-4-
oxopentanedioate (9): An oven dried Biotage $\left.{ }^{\circledR}\right) 2-5 \mathrm{~mL}$ microwave tube
equipped with a stir bar was taken into a nitrogen filled glovebox.
$\left[\mathrm{Cp}^{*} \mathrm{RhCl}_{2}\right]_{2}\left(9.3 \mathrm{mg}, 0.015 \mathrm{mmol}, 0.05\right.$ equiv), $\mathrm{AgSbF}_{6}$ (20.6 $\mathrm{mg}, 0.060$ mmol, 0.2 equiv), AgOAc (10.0 mg, $0.06 \mathrm{mmol}, 0.2$ equiv), $N$-(but-3-en-1-yl)-3,4-dimethoxybenzamide ( $85 \mathrm{mg}, 0.36 \mathrm{mmol}, 1.2$ equiv), and dimethyl $(E)$-4-oxopent-2-enedioate (52 $\mathrm{mg}, 0.30 \mathrm{mmol}, 1$ equiv) were weighed into the reaction vessel. 1,2-Dichloroethane $(1.5 \mathrm{~mL})$ was added, and the vessel was removed from the glovebox and the mixture was stirred under air at $20{ }^{\circ} \mathrm{C}$ for 2.5 hours. The reaction mixture was loaded directly onto a column of silica (pre-equilibrated with a 65:35 hexanes:acetone solvent system), and purification using the 65:35 hexanes:acetone solvent system afforded the product as a pale yellow oil (69 mg, 57\%). Note: approximately 10\% internal alkene isomer of the product co-eluted and was carried through to the next step. ${ }^{1} \mathrm{H}$ NMR $\left(600 \mathrm{MHz}, \mathrm{CDCl}_{3}\right) \delta 6.96(\mathrm{~s}, 1 \mathrm{H}), 6.71(\mathrm{~s}, 1 \mathrm{H}), 6.50(\mathrm{t}$, $J=5.8 \mathrm{~Hz}, 1 \mathrm{H}), 5.84(\mathrm{ddt}, J=17.1,10.2,6.8 \mathrm{~Hz}, 1 \mathrm{H}), 5.16(\mathrm{dq}, J=17.0,1.6 \mathrm{~Hz}, 1 \mathrm{H}), 5.11(\mathrm{dt}, J=10.3$, $1.4 \mathrm{~Hz}, 1 \mathrm{H}), 4.61$ (dd, $J=9.7,4.6 \mathrm{~Hz}, 1 \mathrm{H}), 3.88-3.86$ (m, 9H), 3.77 (dd, $J=19.2,9.8 \mathrm{~Hz}, 1 \mathrm{H}), 3.68$ (s, $3 \mathrm{H}), 3.58(\mathrm{dq}, J=12.3,6.5 \mathrm{~Hz}, 1 \mathrm{H}), 3.50(\mathrm{dq}, J=12.3,6.5 \mathrm{~Hz}, 1 \mathrm{H}), 3.24(\mathrm{dd}, J=19.2,4.5 \mathrm{~Hz}, 1 \mathrm{H}), 2.40$ 
(qd, $J=6.7,3.4 \mathrm{~Hz}, 2 \mathrm{H}) ;{ }^{13} \mathrm{C} \mathrm{NMR}\left(151 \mathrm{MHz}, \mathrm{CDCl}_{3}\right) \delta 191.68,173.30,168.78,160.75,150.67,148.40$, 135.47, 129.33, 127.71, 117.45, 111.13, 110.06, 56.23, 56.17, 53.28, 52.84, 43.25, 41.94, 39.21, 33.87. IR (neat) $\mathrm{cm}^{-1}: 3369,2954,1728,1639,1510,1225,1052,728$. HRMS (ESI) calculated for $\left[\mathrm{C}_{20} \mathrm{H}_{25} \mathrm{NO}_{8} \mathrm{Na}^{+}\right.$ requires $m / z 430.1472$, found 430.1468 .

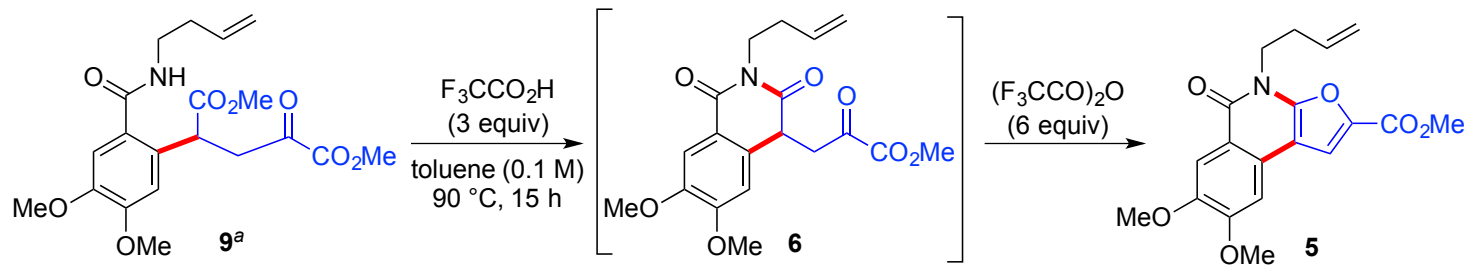

Methyl
$c$-isoquinoline-2-carboxylate (5): Amide 9 (62 mg, $0.15 \mathrm{mmol}, 1$ equiv) was
transferred to a Biotage ${ }^{\circledR} 2-5 \mathrm{~mL}$ microwave tube equipped with a stir bar. The
tube was sealed with a rubber septum and evacuated and back-filled with nitrogen $3 \mathrm{x}$ through a needle inlet. Toluene $(1.5 \mathrm{~mL})$ was added via syringe. Trifluoroacetic acid $(0.04$ $\mathrm{mL}, 0.46 \mathrm{mmol}, 3$ equiv) was added via syringe. The nitrogen inlet was removed and the septum seal was reinforced with electrical tape. The reaction vessel was heated to $90{ }^{\circ} \mathrm{C}$ with stirring for 15 hours. The reaction mixture was allowed to cool to room temperature, the electrical tape was removed, and the nitrogen inlet was replaced. Trifluoroacetic anhydride $(0.13 \mathrm{~mL}, 0.91 \mathrm{mmol}, 6$ equiv) was added via syringe. The nitrogen inlet was again removed and the septum seal was reinforced with electrical tape. The reaction vessel was heated to $90{ }^{\circ} \mathrm{C}$ for 7 hours. The reaction mixture was allowed to cool to room temperature, opened to air, transferred to a vial with dichloromethane and concentrated under a stream of nitrogen. Purification over silica using a 1:1 hexanes:ethyl acetate solvent system afforded the product as a yellow solid $\left(36 \mathrm{mg}, 66 \%\right.$ yield, m.p. $\left.=172-176{ }^{\circ} \mathrm{C}\right)$. Note: approximately $9 \%$ internal alkene isomer of the product co-eluted and was carried through to the next step. ${ }^{1} \mathrm{H}$ NMR $\left(600 \mathrm{MHz}, \mathrm{CDCl}_{3}\right) \delta 7.83(\mathrm{~s}$, 1H), 7.69 (s, 1H), 7.08 (s, 1H), $5.88(\mathrm{ddt}, J=17.1,10.2,7.0 \mathrm{~Hz}, 1 \mathrm{H}), 5.05$ (dq, $J=17.1,1.6 \mathrm{~Hz}, 1 \mathrm{H}), 5.02$ - $4.99(\mathrm{~m}, 1 \mathrm{H}), 4.42(\mathrm{t}, J=7.2 \mathrm{~Hz}, 2 \mathrm{H}), 4.04(\mathrm{~s}, 3 \mathrm{H}), 4.01(\mathrm{~s}, 3 \mathrm{H}), 3.94(\mathrm{~s}, 3 \mathrm{H}), 2.62(\mathrm{q}, J=7.1 \mathrm{~Hz}, 3 \mathrm{H})$; ${ }^{13} \mathrm{C}$ NMR $\left(151 \mathrm{MHz} \mathrm{CDCl}_{3}\right) \delta 160.70,159.16,154.31,150.32,148.70,139.22,134.27,126.42,117.92$, $117.27,114.65,109.80,102.99,101.56,56.39,56.34,52.15,42.02,32.80$. IR (neat) $\mathrm{cm}^{-1}: 2952,1719$, $1651,1570,1254,1087,753$. HRMS (ESI) calculated for $\left[\mathrm{C}_{19} \mathrm{H}_{19} \mathrm{NO}_{6} \mathrm{Na}\right]^{+}$requires $\mathrm{m} / z 380.1105$, found 380.1100 . 

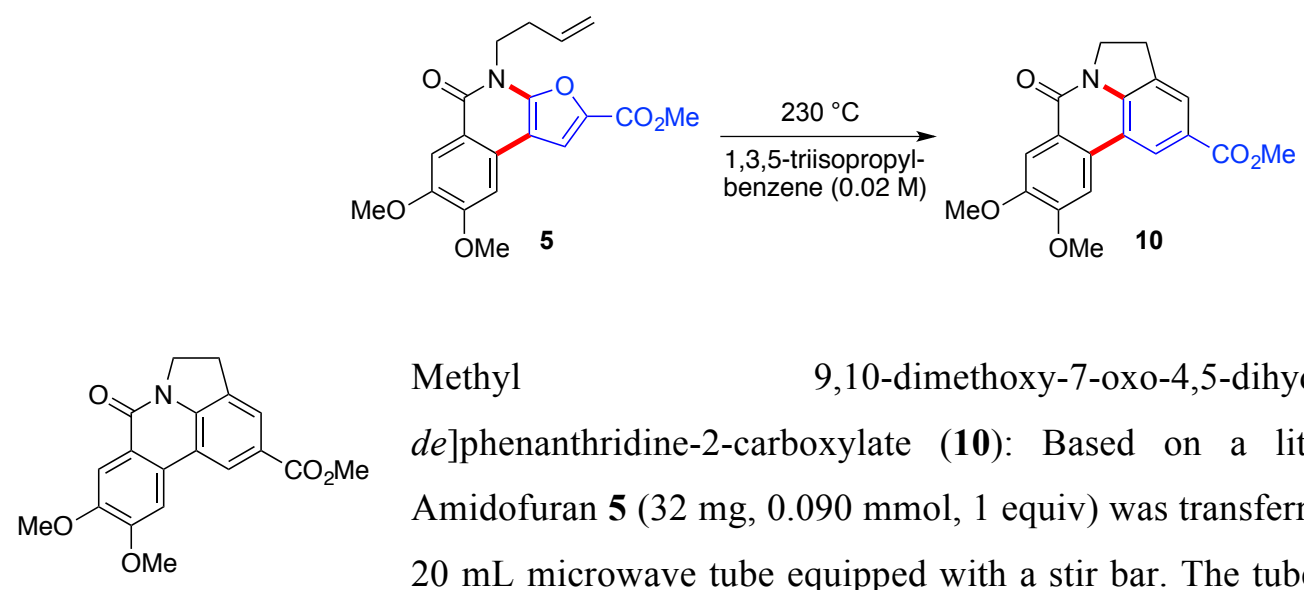

Methyl

9,10-dimethoxy-7-oxo-4,5-dihydro-7H-pyrrolo[3,2,1de]phenanthridine-2-carboxylate (10): Based on a literature procedure, ${ }^{11}$ Amidofuran 5 (32 mg, $0.090 \mathrm{mmol}, 1$ equiv) was transferred to a Biotage ${ }^{\circledR} 10$ $20 \mathrm{~mL}$ microwave tube equipped with a stir bar. The tube was sealed with the appropriate microwave tube cap and evacuated and back-filled with nitrogen $3 \mathrm{x}$ through a needle inlet. 1,3,5-Triisopropylbenzene was passed through a plug of silica, stored under nitrogen in a schlenk flask with activated $3 \AA$ molecular sieves, and degassed under vacuum prior to use. 1,3,5-Triisopropylbenzene $(4.5 \mathrm{~mL})$ was added to the reaction vessel via syringe. The nitrogen inlet was removed and the cap seal was reinforced with electrical tape. The reaction vessel was submerged in a pre-equilibrated sand bath at $230{ }^{\circ} \mathrm{C}$ with stirring for 18 hours. Solid product precipitated on the walls of the microwave tube. The reaction mixture was allowed to cool partially, and the microwave tube cap was removed. The solid product on the walls of the vessel was redissolved in the warm solvent with agitation. While still warm, the reaction mixture was loaded directly onto a column of silica equilibrated with toluene, and toluene was used to transfer the material onto the column. The 1,3,5-triisopropylbenzene was removed by eluting with toluene, and then the solvent system was ramped to $6: 4$ toluene:acetone to afford the product as a white solid $\left(23 \mathrm{mg}, 76 \%\right.$ yield, m.p. $\left.=258-561{ }^{\circ} \mathrm{C}\right)$. The characterization data was consistent with previously reported data. ${ }^{12}{ }^{1} \mathrm{H}$ NMR $\left(600 \mathrm{MHz}, \mathrm{CDCl}_{3}\right) \delta 8.48(\mathrm{~s}, 1 \mathrm{H}), 7.91(\mathrm{~s}, 1 \mathrm{H}), 7.86(\mathrm{~s}, 1 \mathrm{H}), 7.51(\mathrm{~s}$, $1 \mathrm{H}), 4.48(\mathrm{t}, J=8.3 \mathrm{~Hz}, 2 \mathrm{H}), 4.10(\mathrm{~s}, 3 \mathrm{H}), 4.04(\mathrm{~s}, 3 \mathrm{H}), 3.98(\mathrm{~s}, 3 \mathrm{H}), 3.42(\mathrm{t}, J=8.2 \mathrm{~Hz}, 2 \mathrm{H}) ;{ }^{13} \mathrm{C} \mathrm{NMR}$ $\left(151 \mathrm{MHz}, \mathrm{CDCl}_{3}\right) \delta 167.20,159.92,153.28,150.10,142.83,131.21,128.11,125.20,124.50,122.46$, $121.32,116.05,108.79,103.15,56.51,56.40,52.40,47.07,27.14$. IR (neat) $\mathrm{cm}^{-1}: 2945,1710,1658$, 1599, 1256, 1203, 763. HRMS (ESI) calculated for $\left[\mathrm{C}_{19} \mathrm{H}_{17} \mathrm{NO}_{5} \mathrm{Na}\right]^{+}$requires $\mathrm{m} / z$ 362.0999, found 362.0995 . 

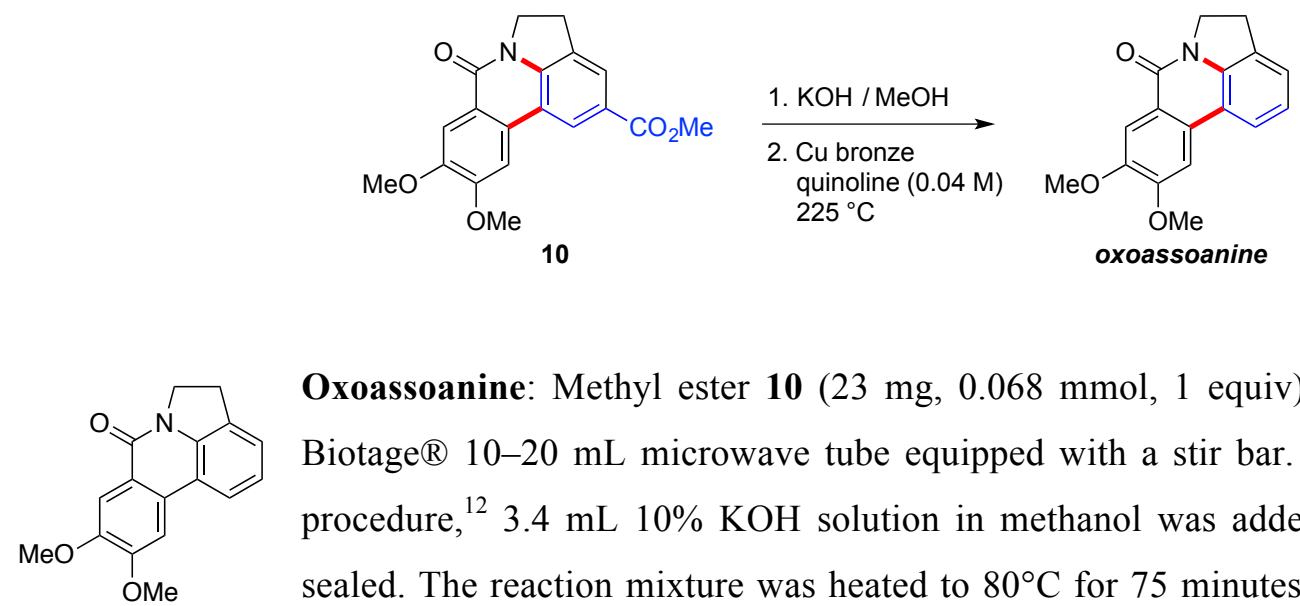

Oxoassoanine: Methyl ester 10 (23 mg, $0.068 \mathrm{mmol}, 1$ equiv) was transferred to a Biotage ${ }^{\circledR} 10-20 \mathrm{~mL}$ microwave tube equipped with a stir bar. Based on a literature procedure, ${ }^{12} 3.4 \mathrm{~mL} 10 \% \mathrm{KOH}$ solution in methanol was added and the vessel was sealed. The reaction mixture was heated to $80^{\circ} \mathrm{C}$ for 75 minutes, then cooled to room temperature and concentrated under a stream of nitrogen. A cold $3 \mathrm{M} \mathrm{HCl}$ solution $(3.4 \mathrm{~mL})$ was added via syringe, and the reaction mixture was stirred at room temperature for 1 hour. The resulting white solids were collected over a Büchner funnel, rinsing with water. The solids were transferred back to the Biotage ${ }^{\circledR}$ 10-20 mL microwave tube equipped with stir bar. Copper bronze (32 mg) was added, and the reaction vessel was sealed and evacuated and back-filled with nitrogen $3 x$ through a needle inlet. Freshly distilled and degassed (freeze-pump-thaw) quinoline $(1.7 \mathrm{~mL}$ ) was added via syringe. The nitrogen inlet was removed and the cap seal was reinforced with electrical tape. The reaction vessel was submerged in a pre-equilibrated sand bath at $225{ }^{\circ} \mathrm{C}$ with stirring for 15 hours. The reaction mixture was allowed to cool to room temperature and then filtered through a plug of celite, eluting with dichloromethane. The reaction mixture was diluted with $50 \mathrm{~mL}$ of dichloromethane and washed $3 \mathrm{x}$ with $3 \mathrm{M} \mathrm{HCl}$, then brine, and then dried over sodium sulfate and concentrated. The residue was purified over silica using a 9:1 dichloromethane:diethyl ether solvent system to afford the product as a white solid $(13 \mathrm{mg}, 68 \%$ yield, m.p. $\left.=267-270{ }^{\circ} \mathrm{C}\right)$. The characterization data was consistent with previously reported data. ${ }^{12}{ }^{1} \mathrm{H}$ NMR $\left(600 \mathrm{MHz}, \mathrm{CDCl}_{3}\right) \delta 7.96(\mathrm{~s}, 1 \mathrm{H}), 7.82(\mathrm{~d}, J=7.9 \mathrm{~Hz}, 1 \mathrm{H}), 7.56(\mathrm{~s}, 1 \mathrm{H}), 7.30(\mathrm{~d}, J=7.2 \mathrm{~Hz}, 1 \mathrm{H}), 7.22(\mathrm{t}$, $J=7.6 \mathrm{~Hz}, 1 \mathrm{H}), 4.50(\mathrm{t}, J=8.2 \mathrm{~Hz}, 2 \mathrm{H}), 4.09(\mathrm{~s}, 3 \mathrm{H}), 4.05(\mathrm{~s}, 3 \mathrm{H}), 3.45(\mathrm{t}, J=8.2 \mathrm{~Hz}, 2 \mathrm{H}) ;{ }^{13} \mathrm{C} \mathrm{NMR}$ $\left(151 \mathrm{MHz}, \mathrm{CDCl}_{3}\right) \delta 159.84,153.04,149.77,139.58,131.07,128.66,123.74,123.33,121.51,119.36$, 116.85, 108.94, 103.10, 56.40, 56.29, 46.65, 27.58. IR (neat) $\mathrm{cm}^{-1}: 2933,1641,1604,1270,1030,756$. HRMS (ESI) calculated for $\left[\mathrm{C}_{17} \mathrm{H}_{15} \mathrm{NO}_{3} \mathrm{Na}\right]^{+}$requires $\mathrm{m} / \mathrm{z} 304.0944$, found 304.0938 . 


\section{References}

1. Fujita, K.; Takahashi, Y.; Owaki, M.; Yamamoto, K.; Yamaguchi, R. Org. Lett. 2004, 6, 2785.

2. Ackermann, L.; Lygin, A. V.; Hofmann, N. Angew. Chem. Int. Ed. 2011, 50, 6379.

3. Wrigglesworth, J. W.; Cox, B.; Lloyd-Jones, G. C.; Booker-Milburn, K. I. Org. Lett. 2011, 13, 5326.

4. Miyoshi, T.; Matsuya, S.; Tsugawa, M.; Sato, S.; Ueda, M.; Miyata, O. Org. Lett. 2013, 13, 3374.

5. Moriarty, R. M.; Berglund, B. A.; Penmasta, R. Tetrahedron Lett. 1992, 33, 6065.

6. Runcie, K. A.; Taylor, R. J. K. Chem. Commun. 2002, 974.

7. Abbasov, M. E.; Hudson, M. B.; Tantillo, D. J.; Romo, D. J. Am. Chem. Soc. 2014, 136, 4492.

8. Arena, G.; Chen, C. C.; Leonori, D.; Aggarwal, V. K. Org. Lett. 2013, 15, 4250.

9. Bazin, S.; Feray, L.; Vanthuyne, N.; Bertrand, M. P. Tetrahedron 2005, 61, 4261.

10. Carrigan, C. N.; Bartlett, R. D.; Esslinger, C. S.; Cybulski, K. A.; Tongcharoensirikul, P.; Bridges, R. J.; Thompson, C. M. J. Med. Chem. 2002, 45, 2260.

11. Wolkenberg, S. E.; Boger, D. L. J. Org. Chem. 2002, 67, 7361.

12. Padwa, A.; Dimitroff, M.; Waterson, A. G.; Wu, T. H. J. Org. Chem. 1998, 63, 3986. 
VII. ${ }^{1} \mathrm{H}$ and ${ }^{13} \mathrm{C}$ NMR spectra

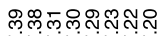

rinivin

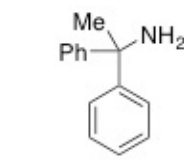

$1 \mathrm{~h}$
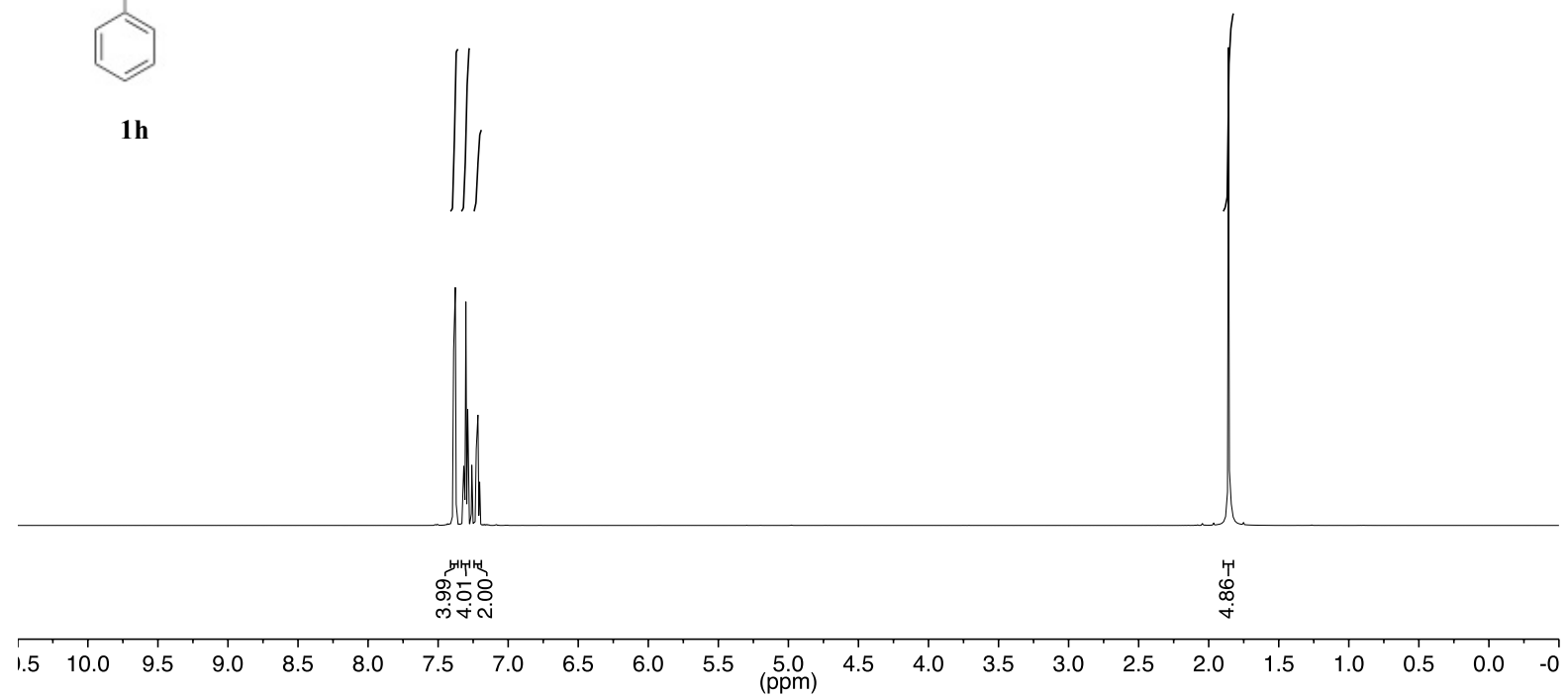

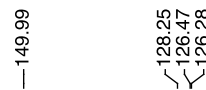
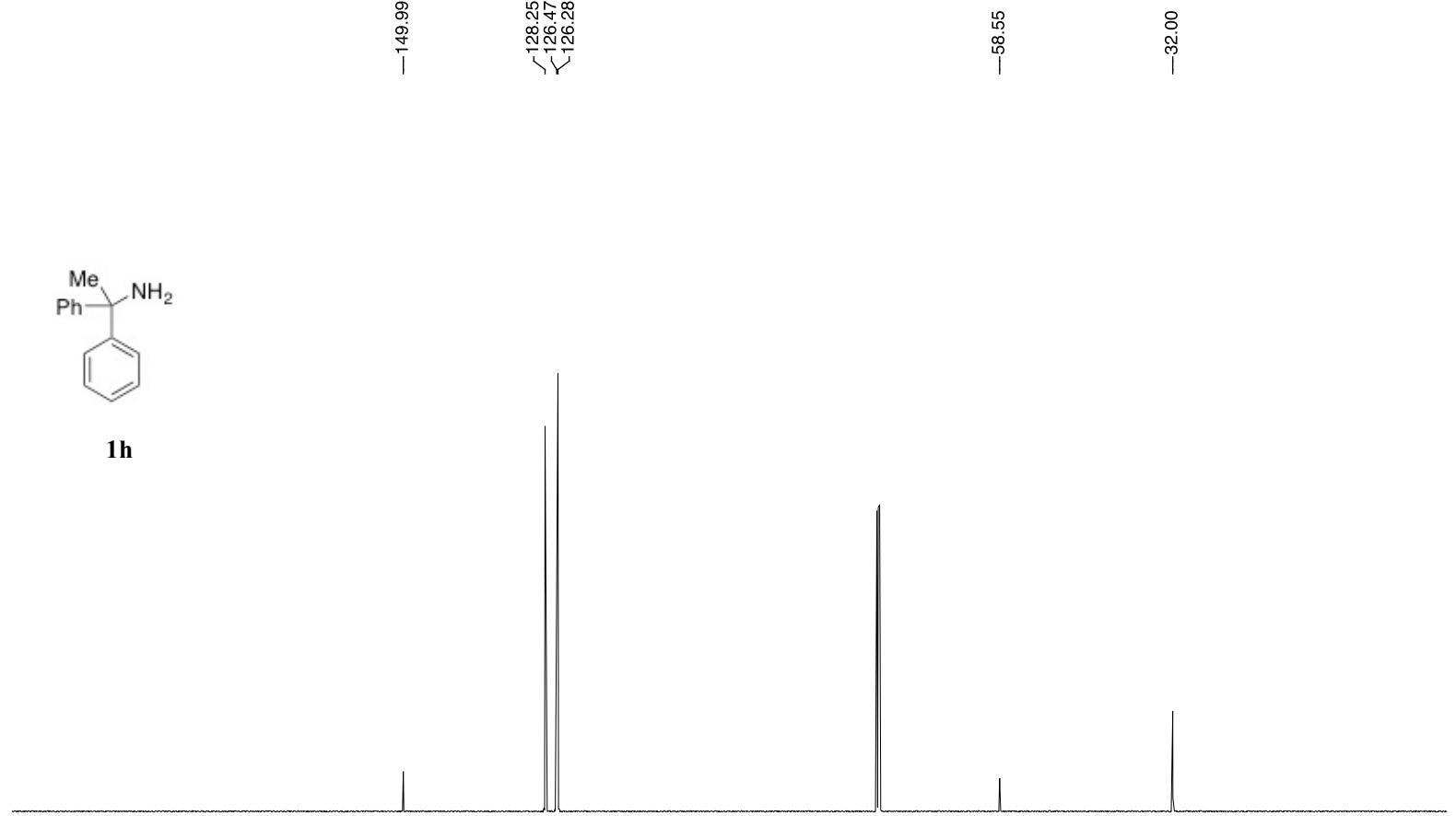

$\begin{array}{lllllllllllllllllllllll}10 & 200 & 190 & 180 & 170 & 160 & 150 & 140 & 130 & 120 & 110 & \begin{array}{c}100 \\ (\mathrm{ppm})\end{array} & 90 & 80 & 70 & 60 & 50 & 40 & 30 & 20 & 10 & 0 & -1\end{array}$ 


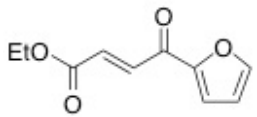

$2 b$

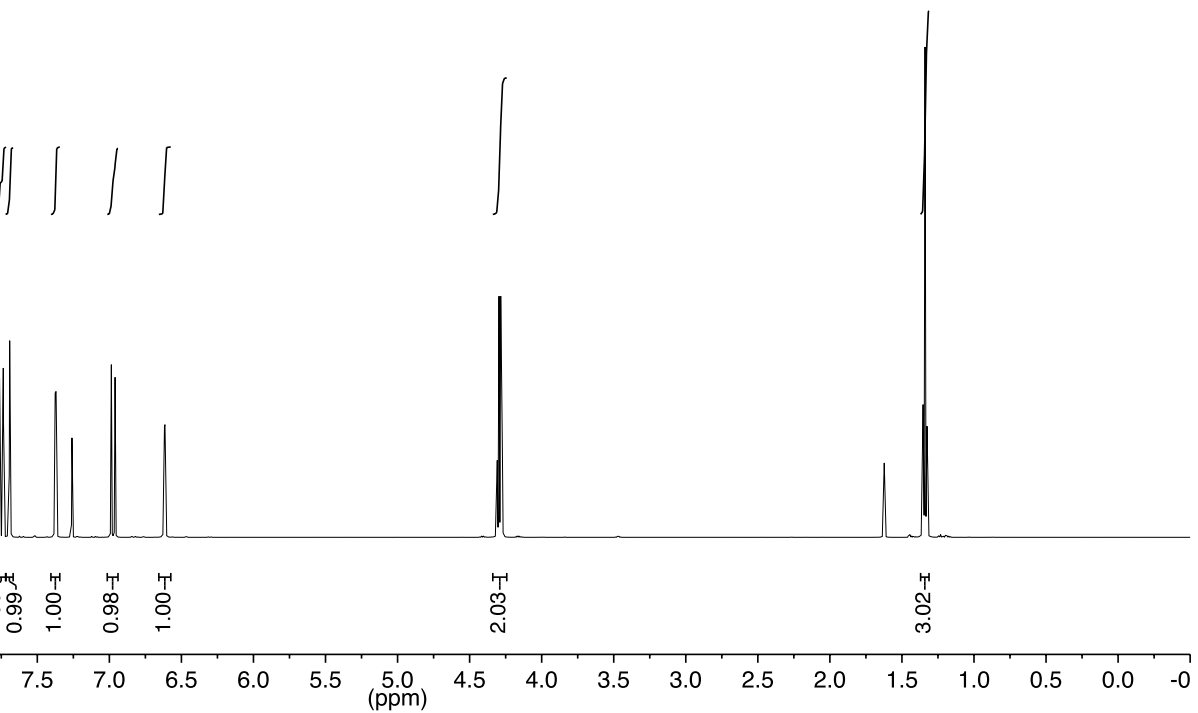

$\underset{\stackrel{\leftrightarrow}{0}}{\stackrel{0}{0}}$

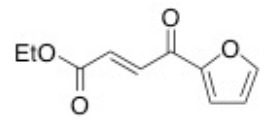

2b

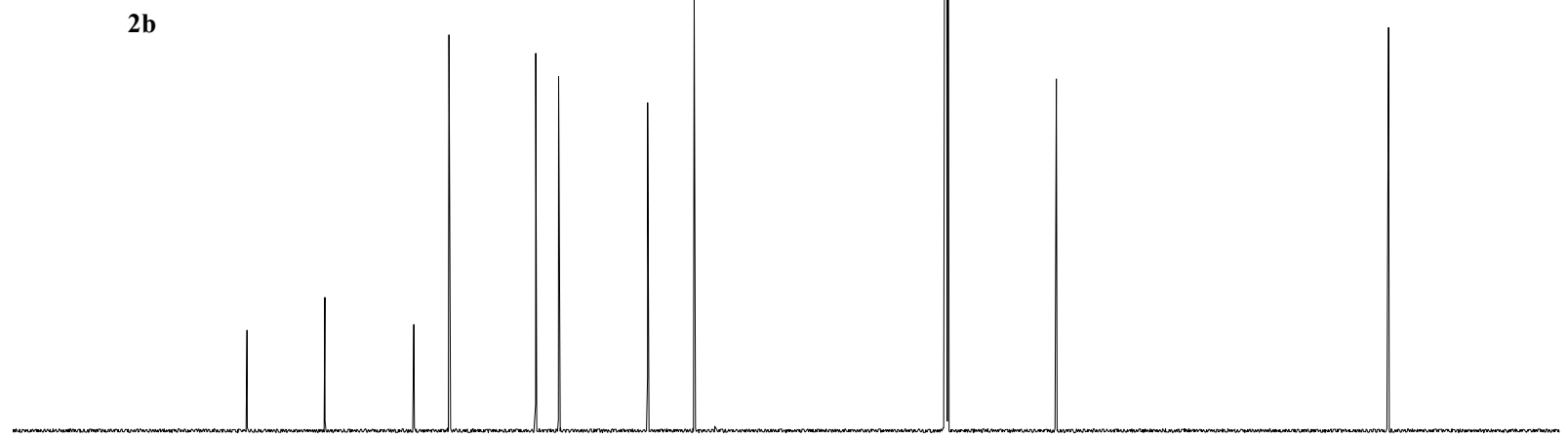

\begin{tabular}{llllllllllllllllllllllll}
\hline 10 & 200 & 190 & 180 & 170 & 160 & 150 & 140 & 130 & 120 & 110 & $\begin{array}{c}100 \\
(\mathrm{ppm})\end{array}$ & 90 & 80 & 70 & 60 & 50 & 40 & 30 & 20 & 10 & 0 & -1
\end{tabular} 

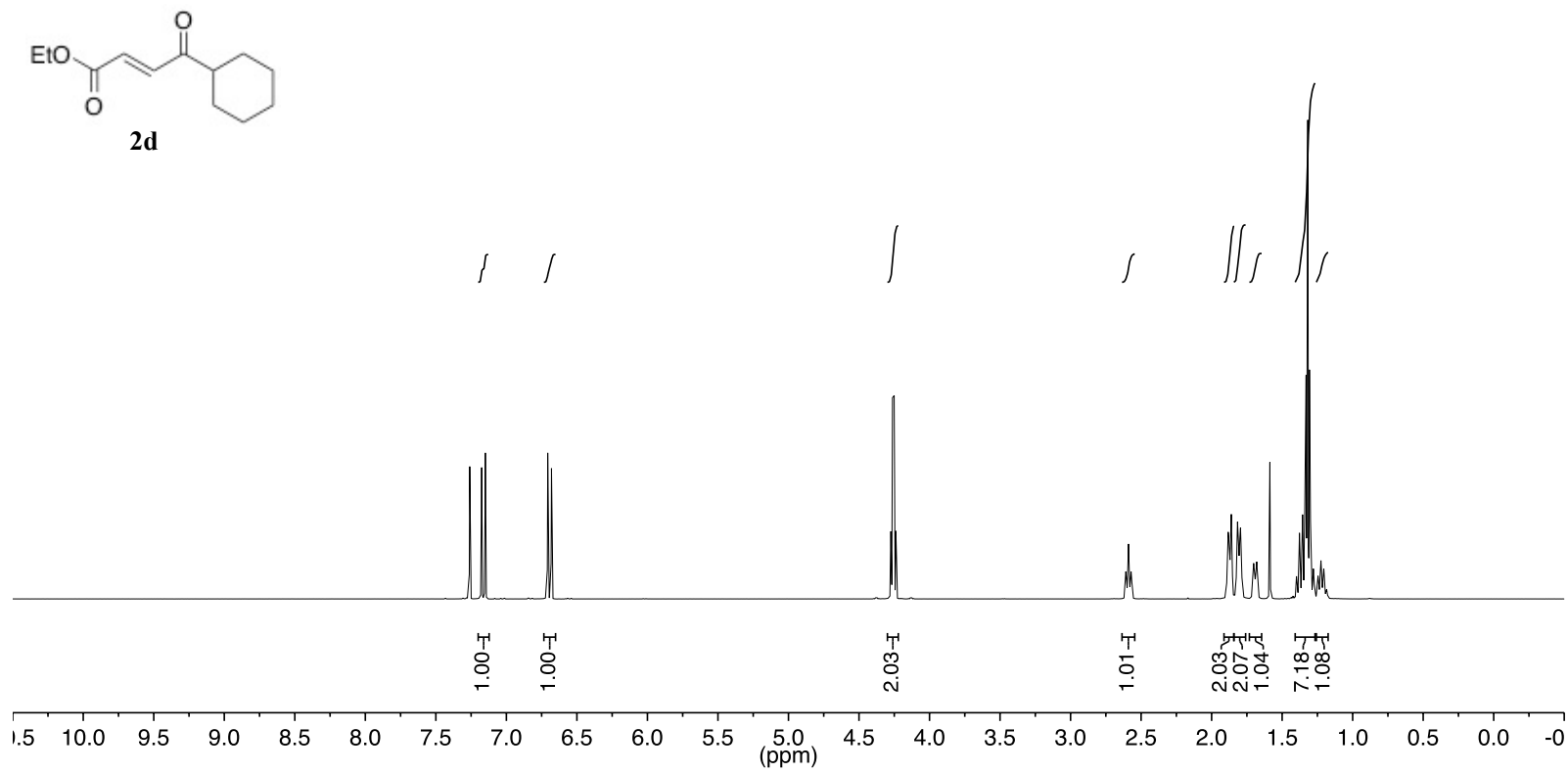

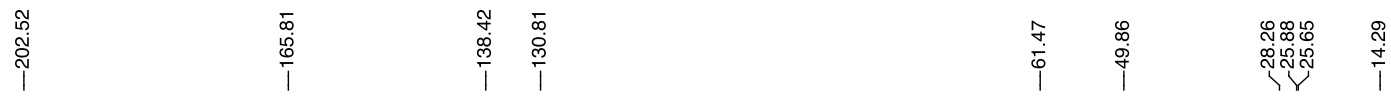
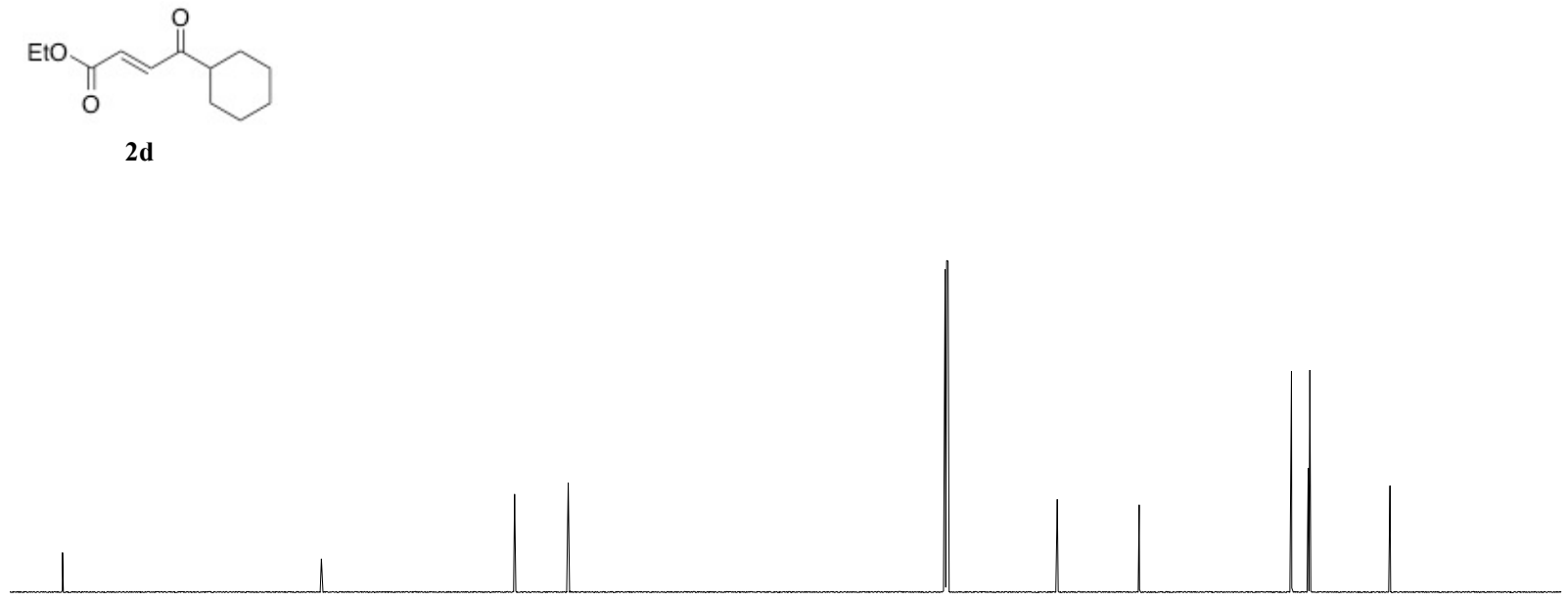

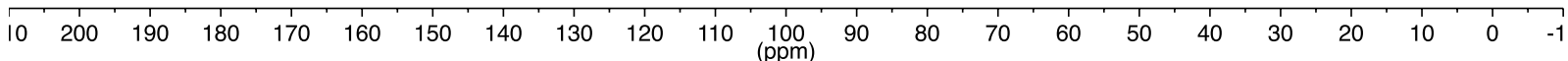




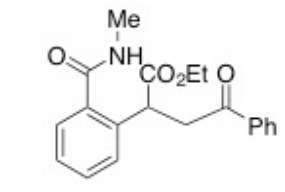

3a
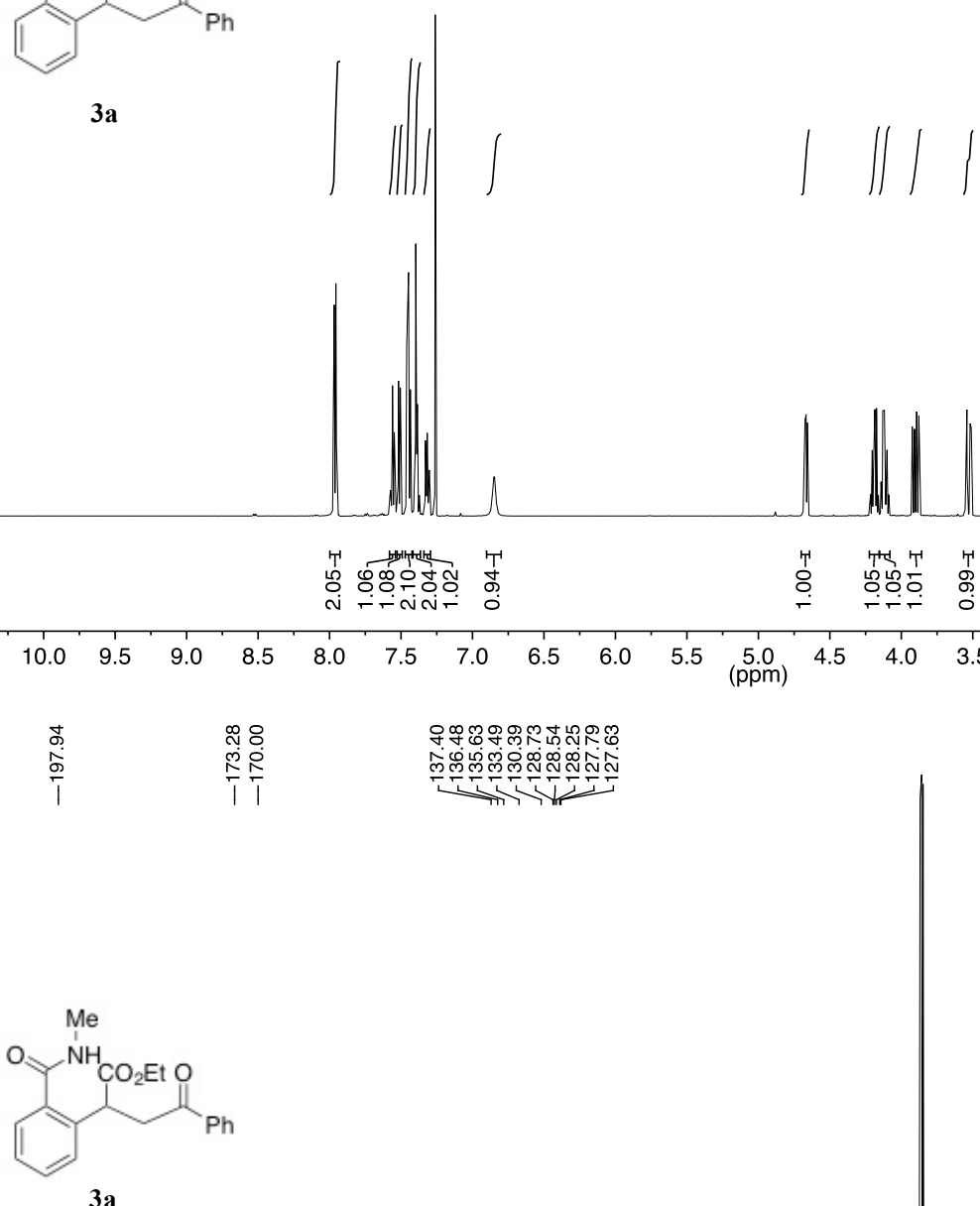

3a

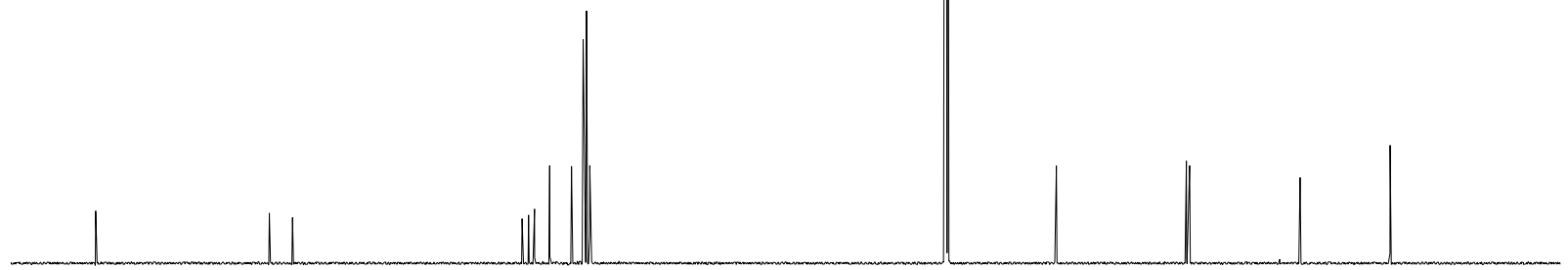

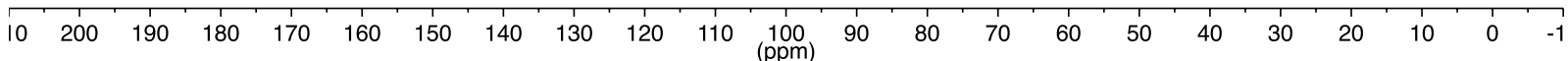




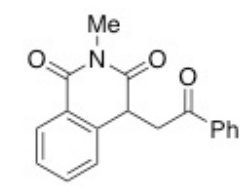

$4 a$
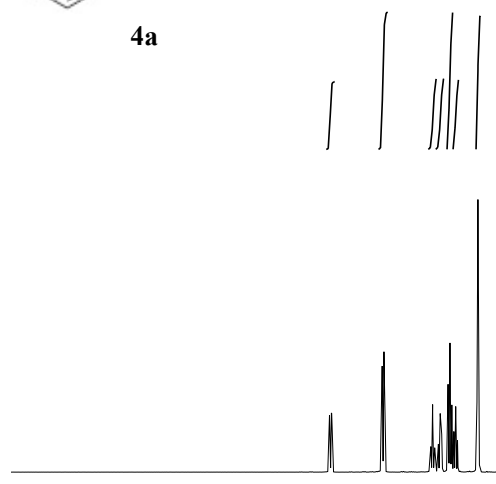

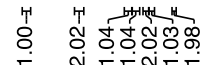

\begin{tabular}{|c|c|c|c|c|c|c|c|c|c|}
\hline 1.5 & 10.0 & 9.5 & 9.0 & 8.5 & 8.0 & 7.5 & 7.0 & 6.5 & 6.0 \\
\hline & $\begin{array}{l}\bar{m} \\
\stackrel{8}{\circ} \\
\stackrel{\rho}{\dagger}\end{array}$ & & & 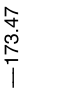 & ְ. & & 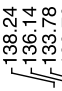 & 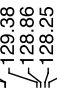 & 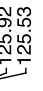 \\
\hline
\end{tabular}

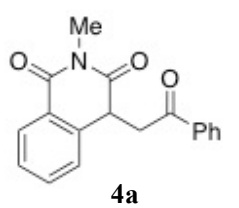

$4 \mathbf{a}$

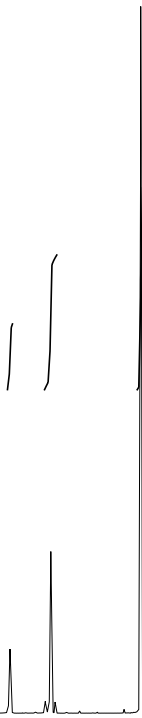

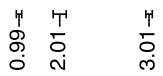

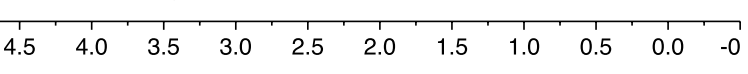

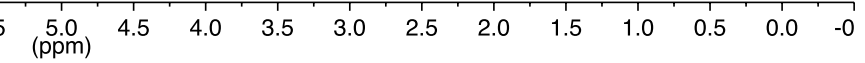

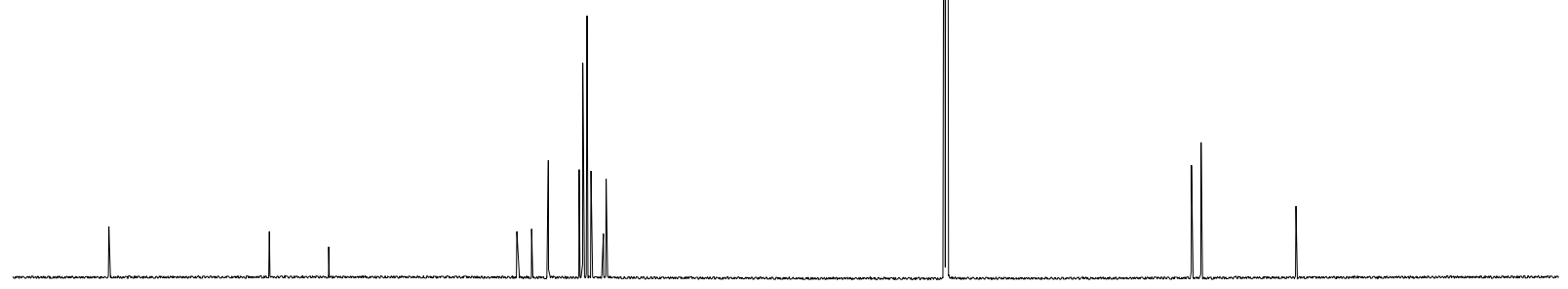

\begin{tabular}{llllllllllllllllllllllll}
\hline 10 & 200 & 190 & 180 & 170 & 160 & 150 & 140 & 130 & 120 & 110 & $\begin{array}{c}100 \\
(\mathrm{ppm})\end{array}$ & 90 & 80 & 70 & 60 & 50 & 40 & 30 & 20 & 10 & 0 & -1
\end{tabular} 

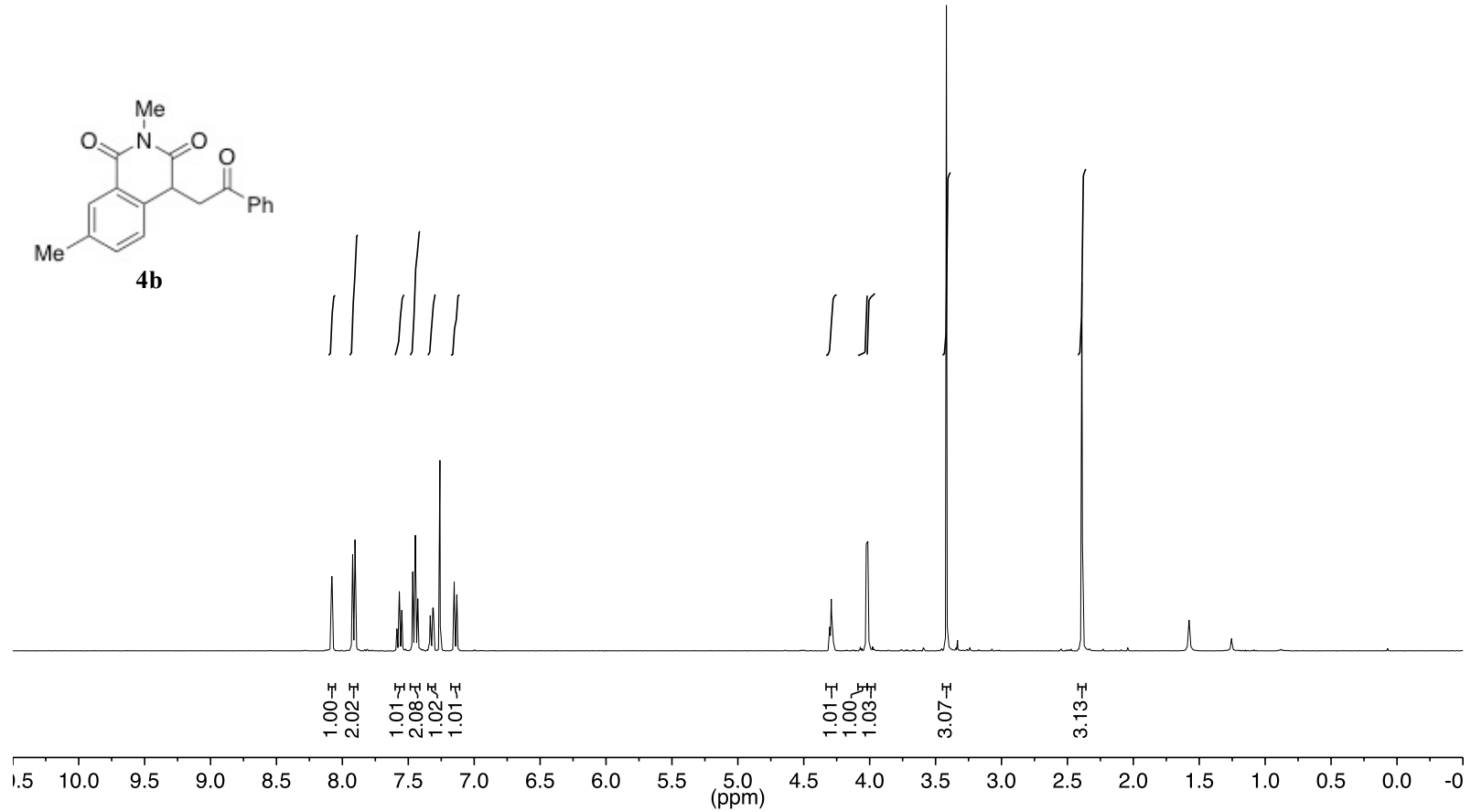

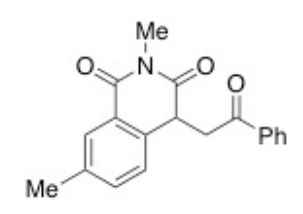

$4 \mathbf{b}$
מ⿱⺈ लَm $5 \div-\div \div$

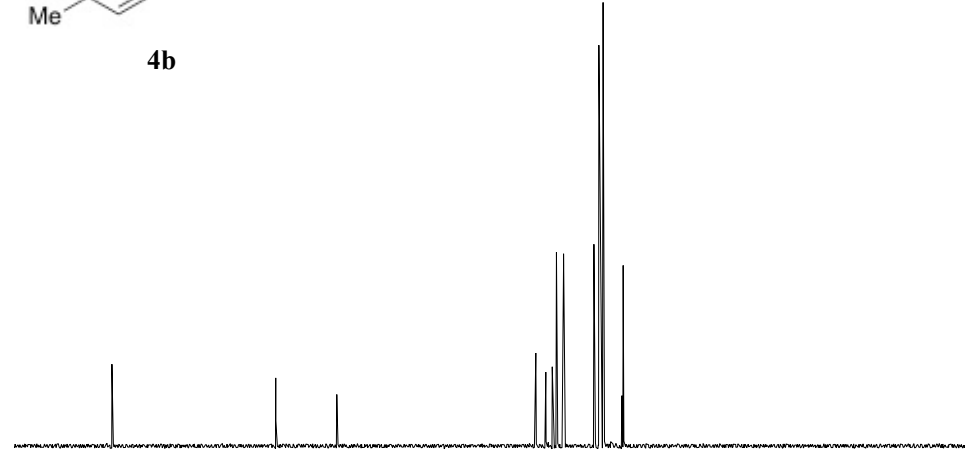

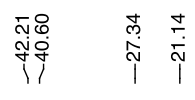

\begin{tabular}{llllllllllllllllllllllll}
\hline 10 & 200 & 190 & 180 & 170 & 160 & 150 & 140 & 130 & 120 & 110 & $\begin{array}{c}100 \\
(\mathrm{ppm})\end{array}$ & 90 & 80 & 70 & 60 & 50 & 40 & 30 & 20 & 10 & 0 & -1
\end{tabular} 


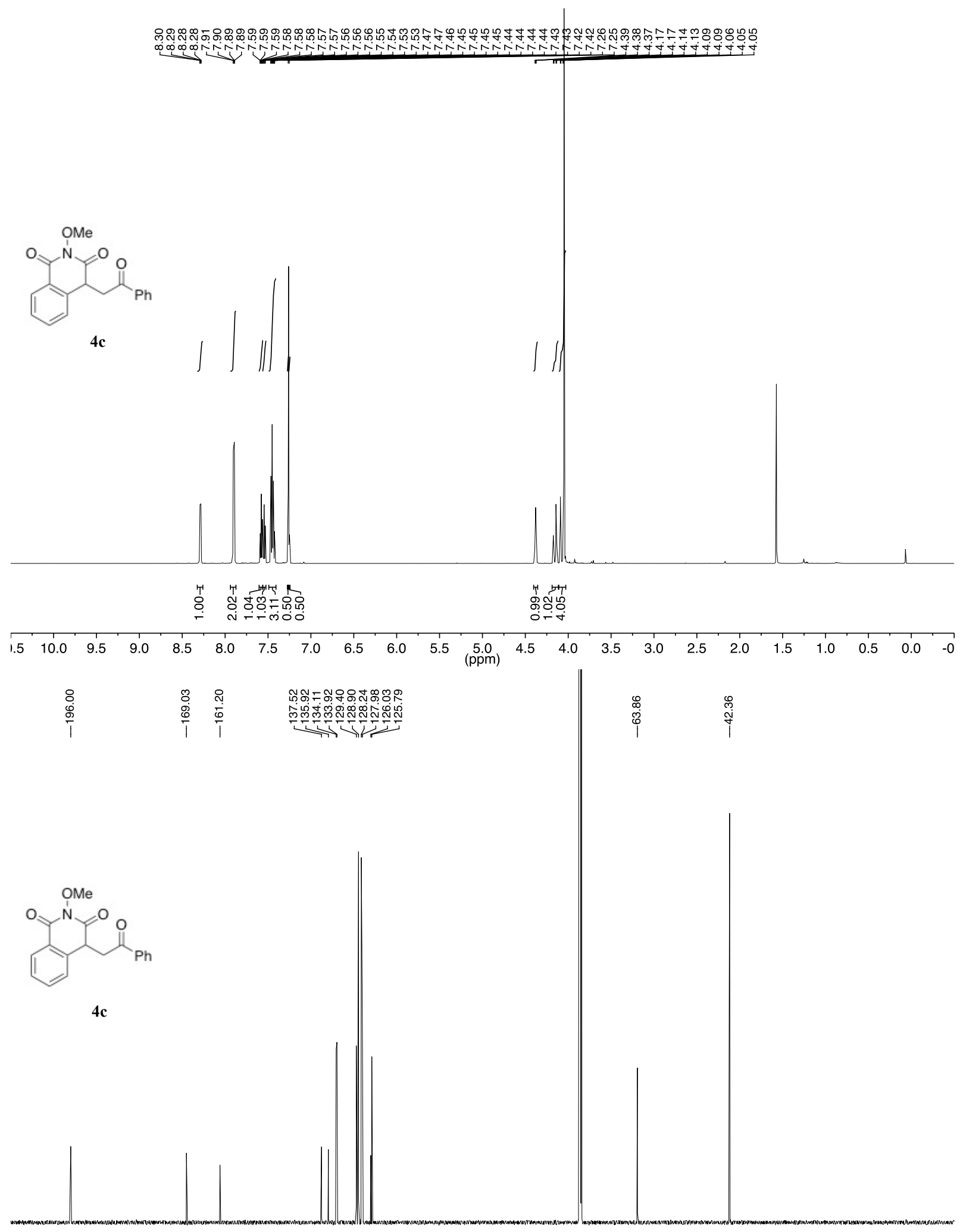

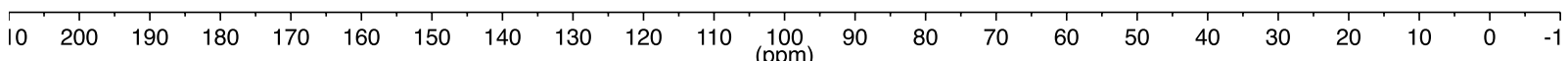



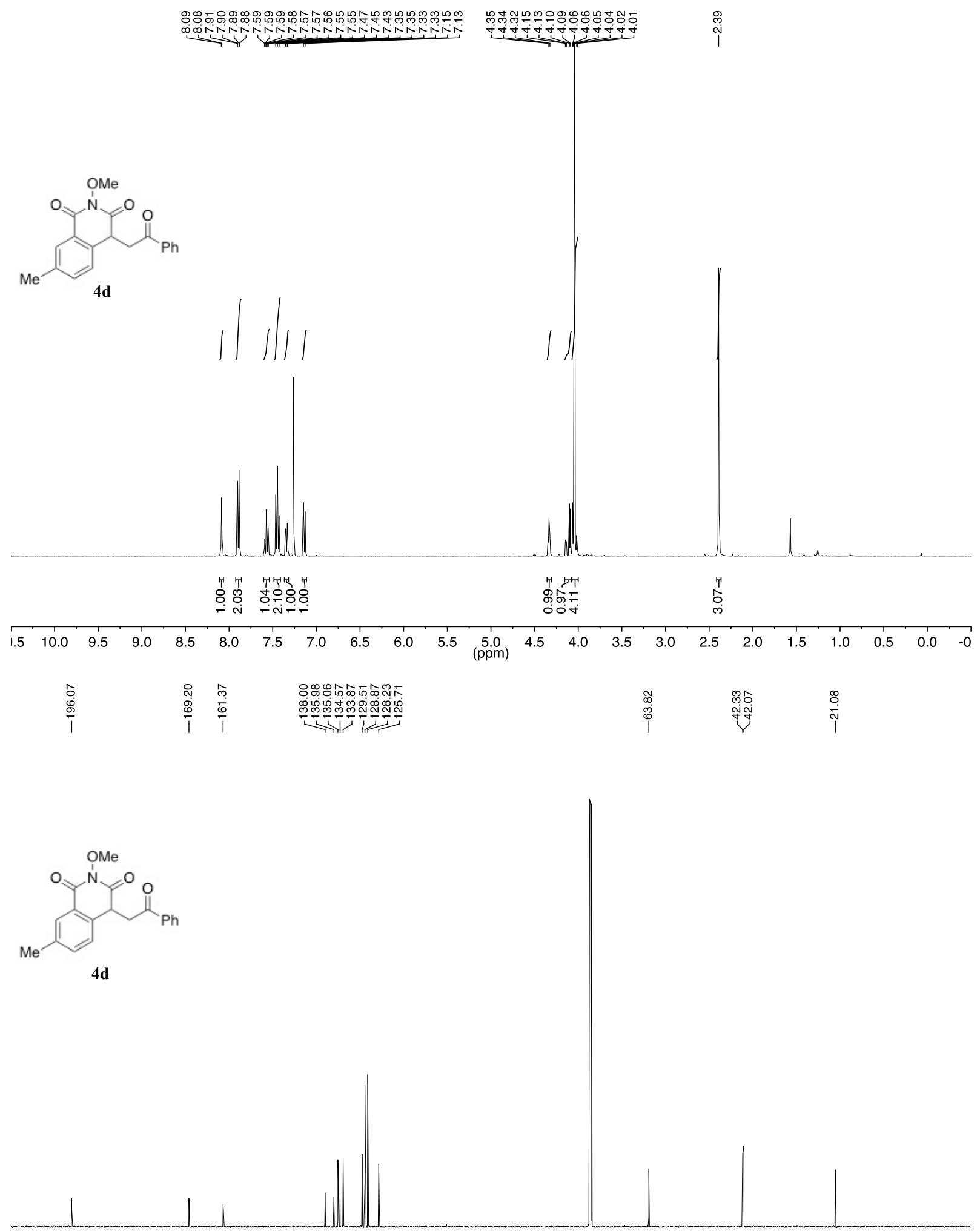

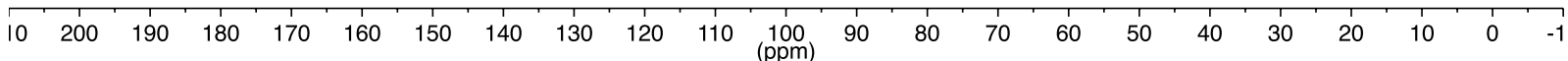




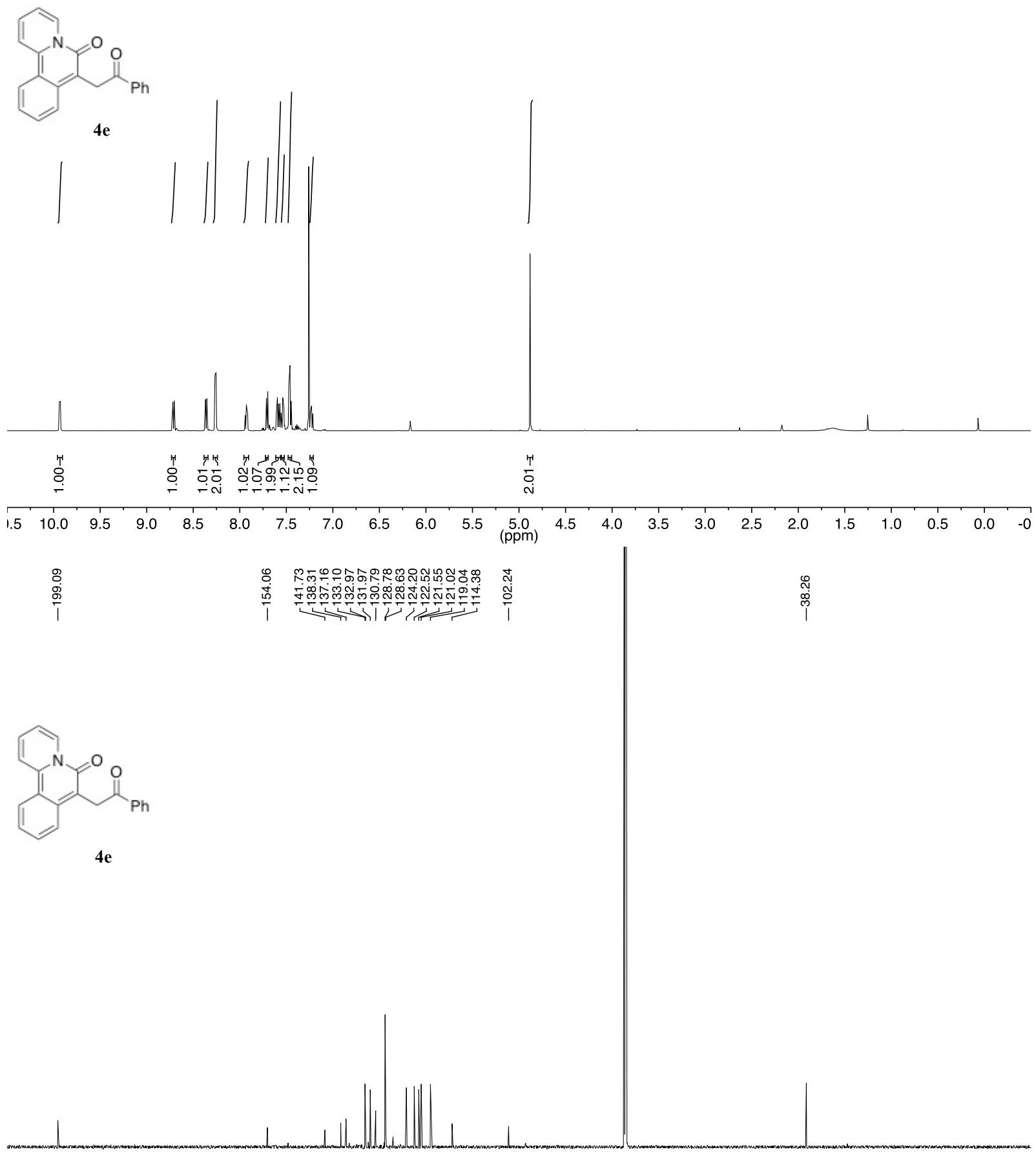

$\begin{array}{lllllllllllllllllllllllllllllllll}10 & 200 & 190 & 180 & 170 & 160 & 150 & 140 & 130 & 120 & 110 & \begin{array}{c}100 \\ \mathrm{ppm})\end{array} & 90 & 80 & 70 & 60 & 50 & 40 & 30 & 20 & 10 & 0 & -1\end{array}$ 

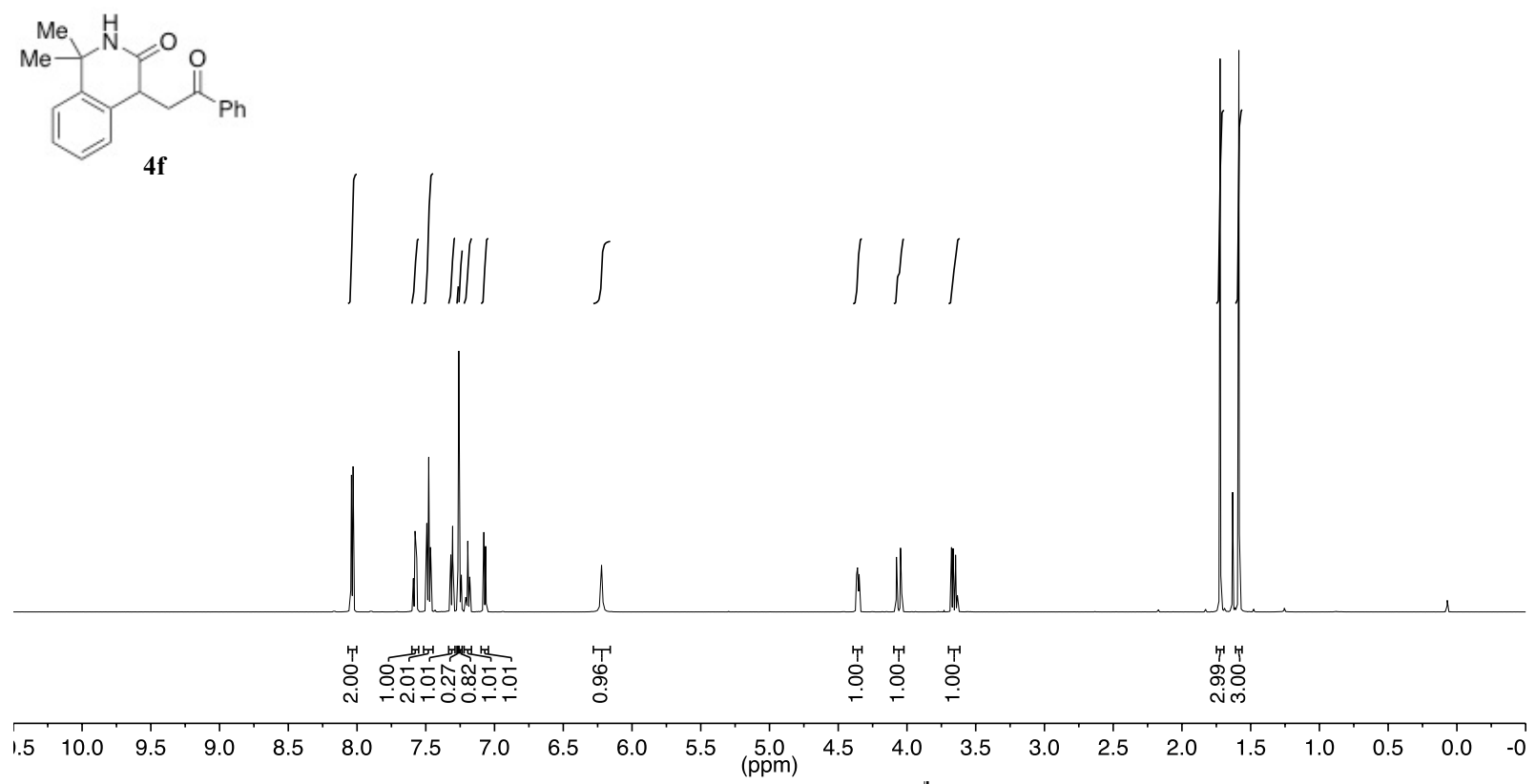

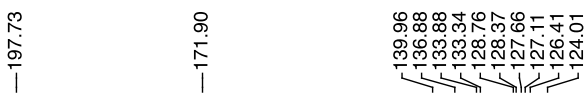

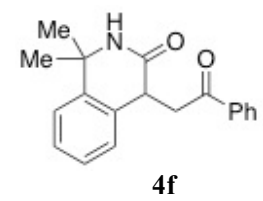

$4 \mathbf{f}$
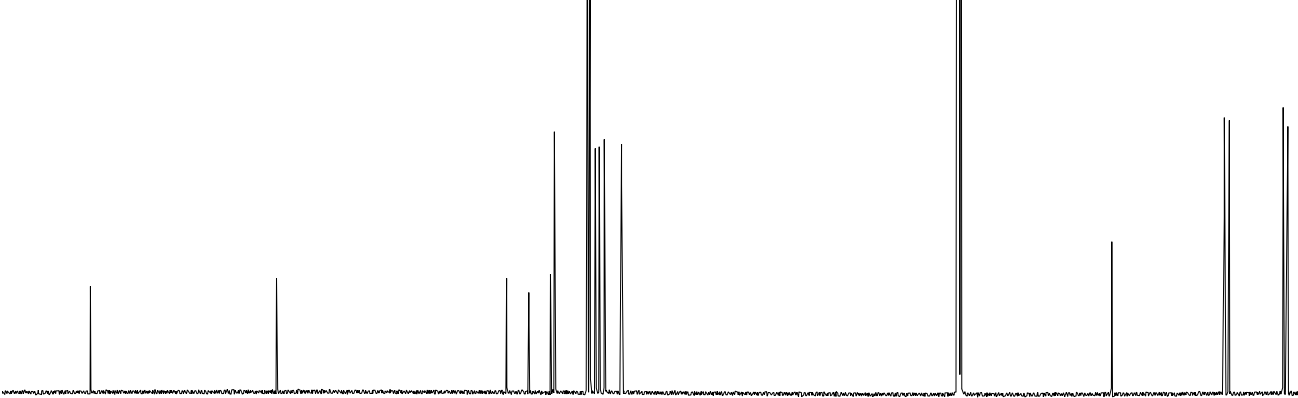

\begin{tabular}{lllllllllllllllllllllll}
\hline 10 & 200 & 190 & 180 & 170 & 160 & 150 & 140 & 130 & 120 & 110 & $\begin{array}{c}100 \\
(\mathrm{ppm})\end{array}$ & 90 & 80 & 70 & 60 & 50 & 40 & 30 & 20 & 10 & 0 & -1
\end{tabular} 


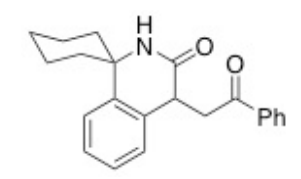

$4 \mathrm{~g}$

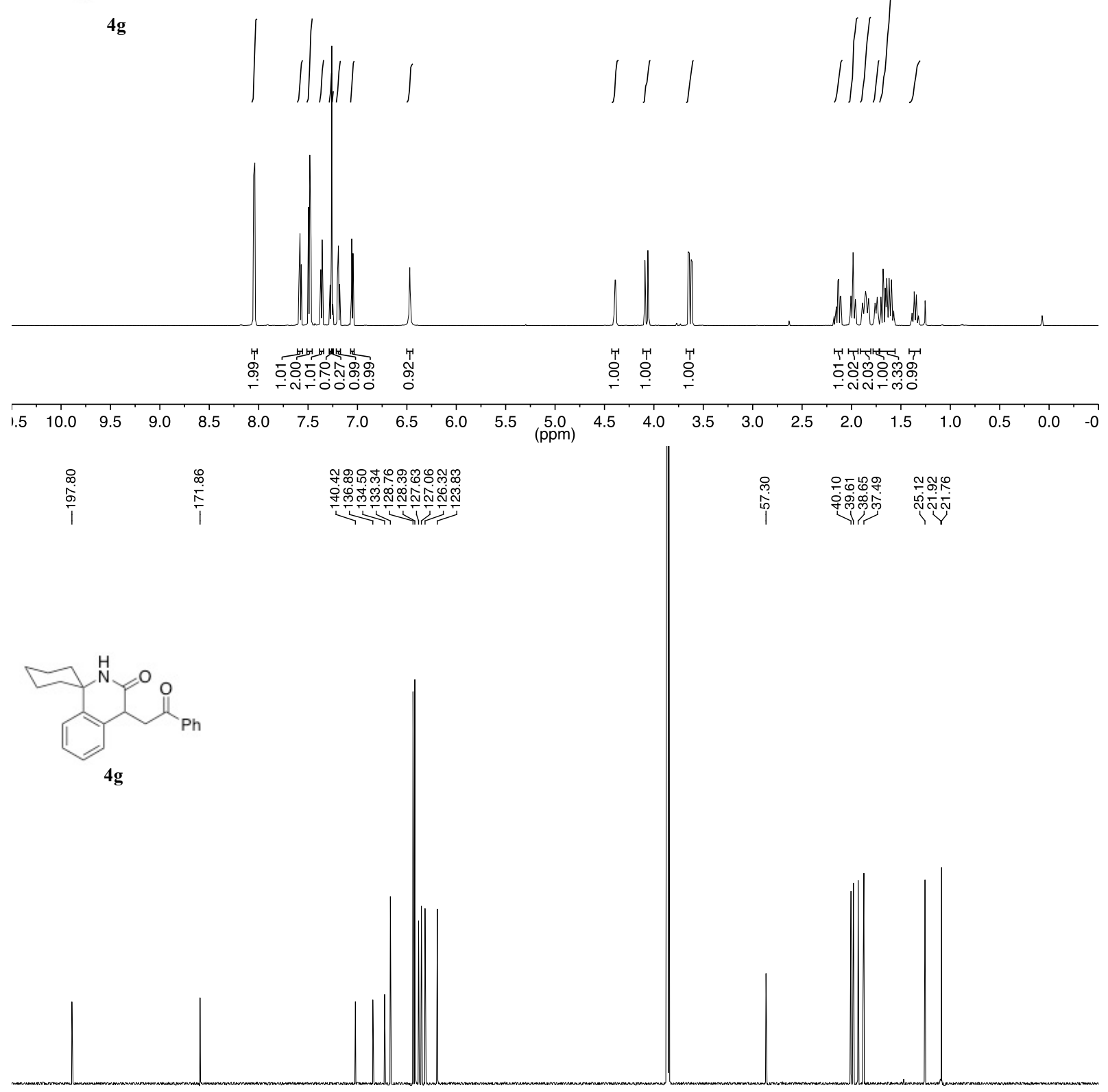

\begin{tabular}{lllllllllllllllllllllll}
\hline 10 & 200 & 190 & 180 & 170 & 160 & 150 & 140 & 130 & 120 & 110 & $\begin{array}{c}100 \\
(\mathrm{ppm})\end{array}$ & 90 & 80 & 70 & 60 & 50 & 40 & 30 & 20 & 10 & 0 & -1
\end{tabular} 

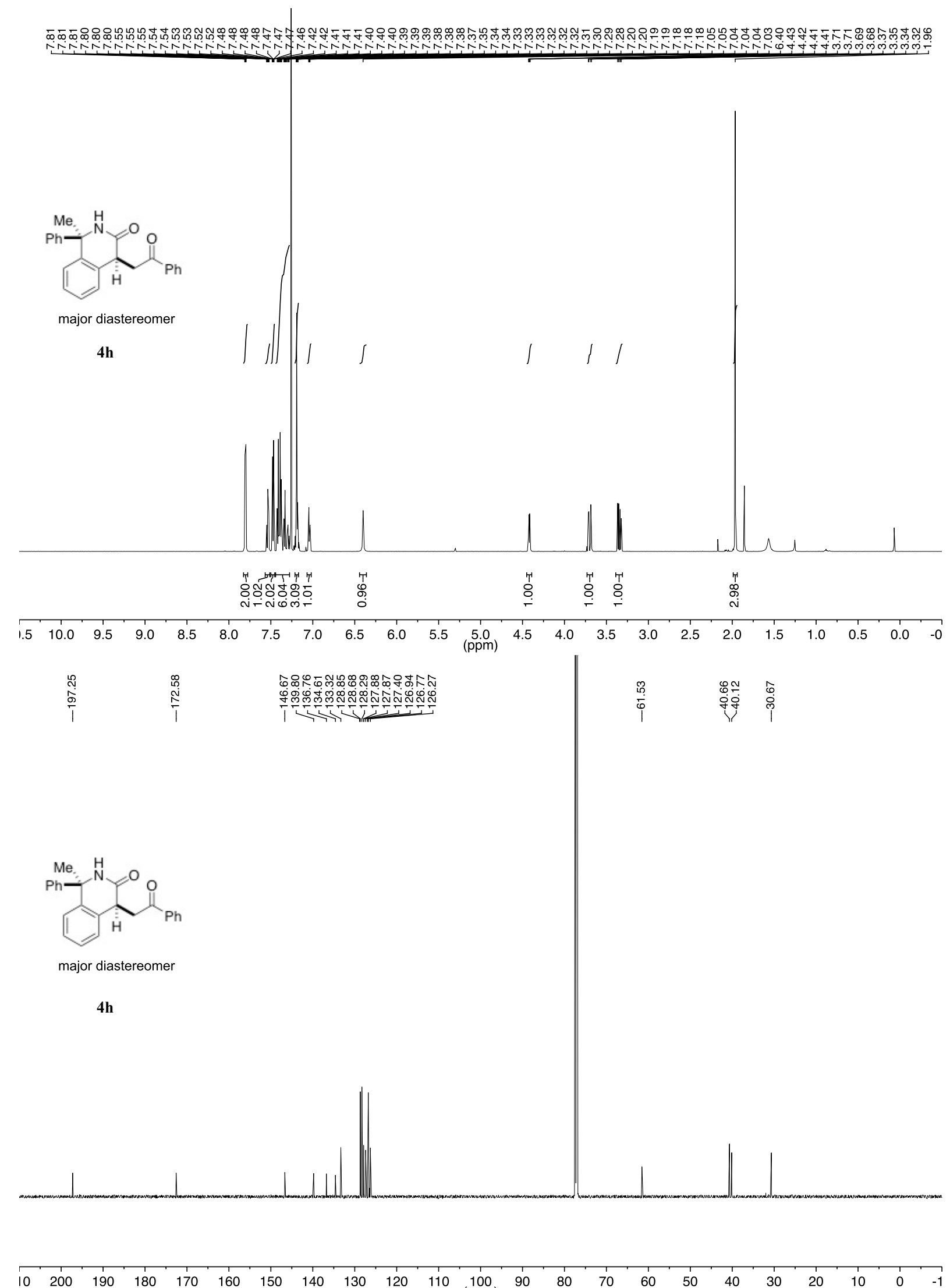

$\begin{array}{llllllllllll}10 & 200 & 190 & 180 & 170 & 160 & 150 & 140 & 130 & 120 & 110 & \begin{array}{c}100 \\ (\mathrm{ppm})\end{array}\end{array}$

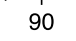

8070 


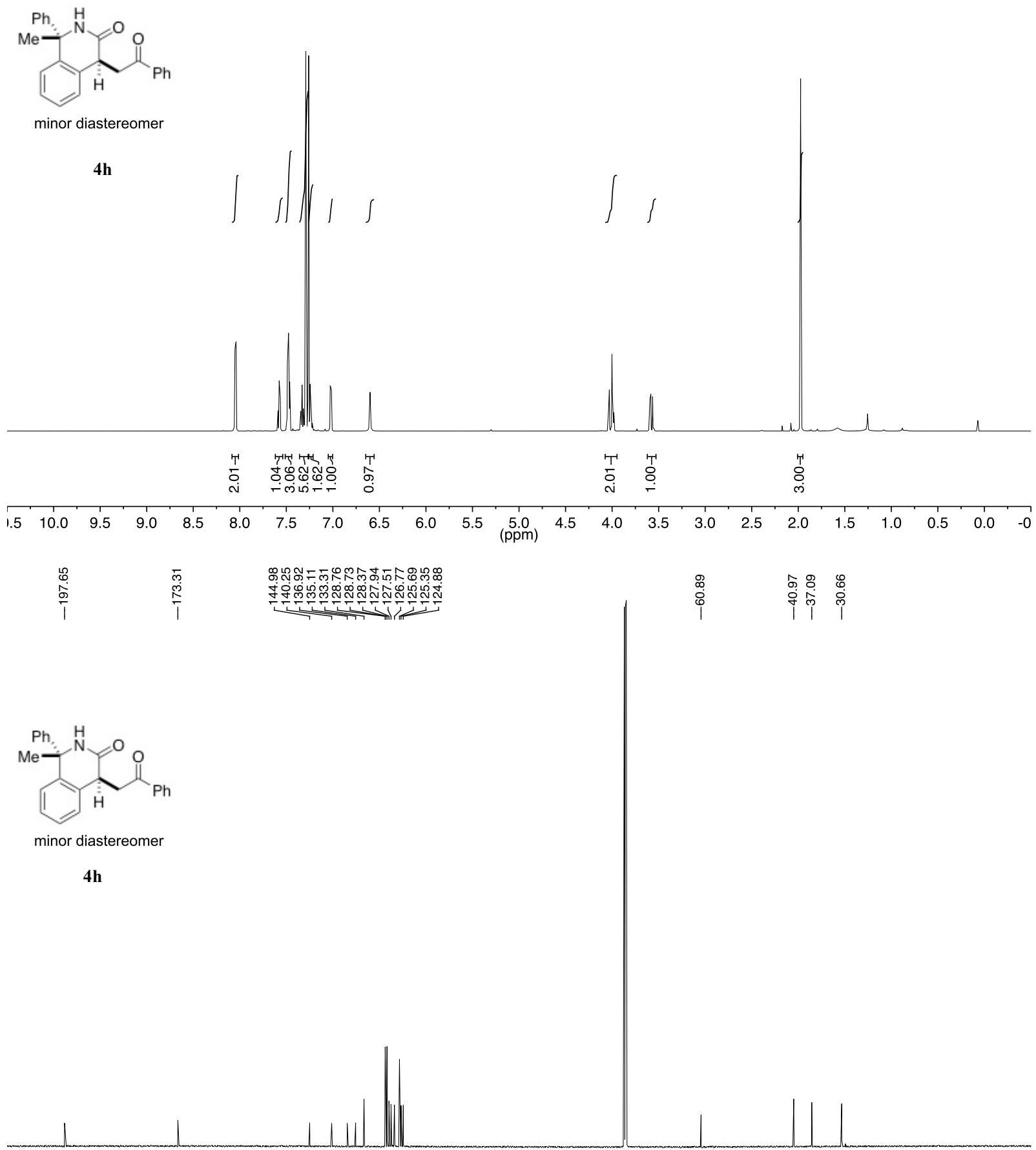

\begin{tabular}{llllllllllllllllllllllll}
\hline 10 & 200 & 190 & 180 & 170 & 160 & 150 & 140 & 130 & 120 & 110 & $\begin{array}{c}100 \\
(\mathrm{ppm})\end{array}$ & 90 & 80 & 70 & 60 & 50 & 40 & 30 & 20 & 10 & 0 & -1
\end{tabular} 

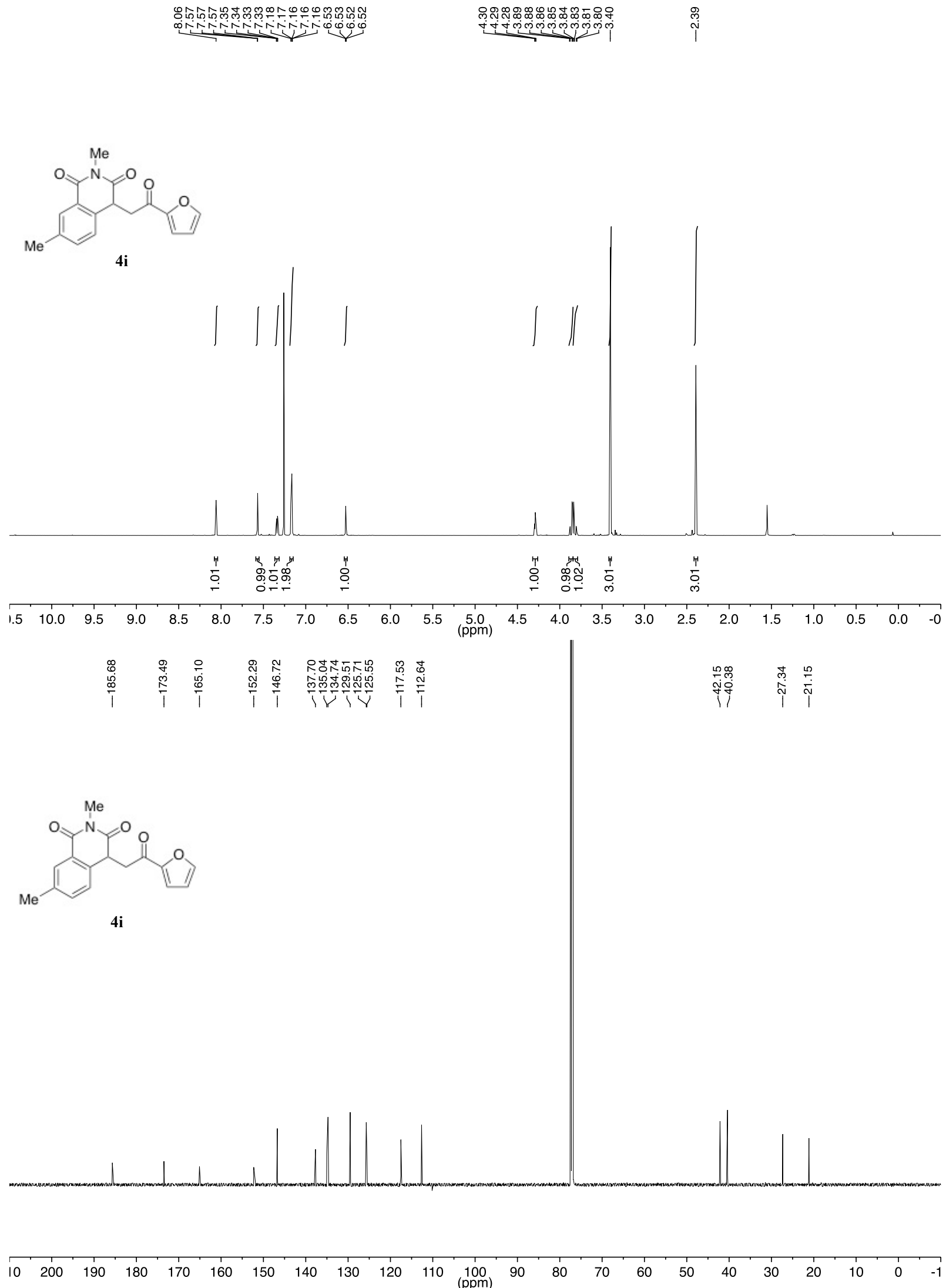


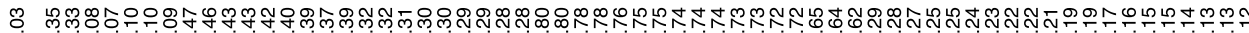
$\underbrace{0}_{0}$

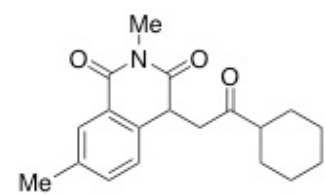

$4 \mathbf{j}$

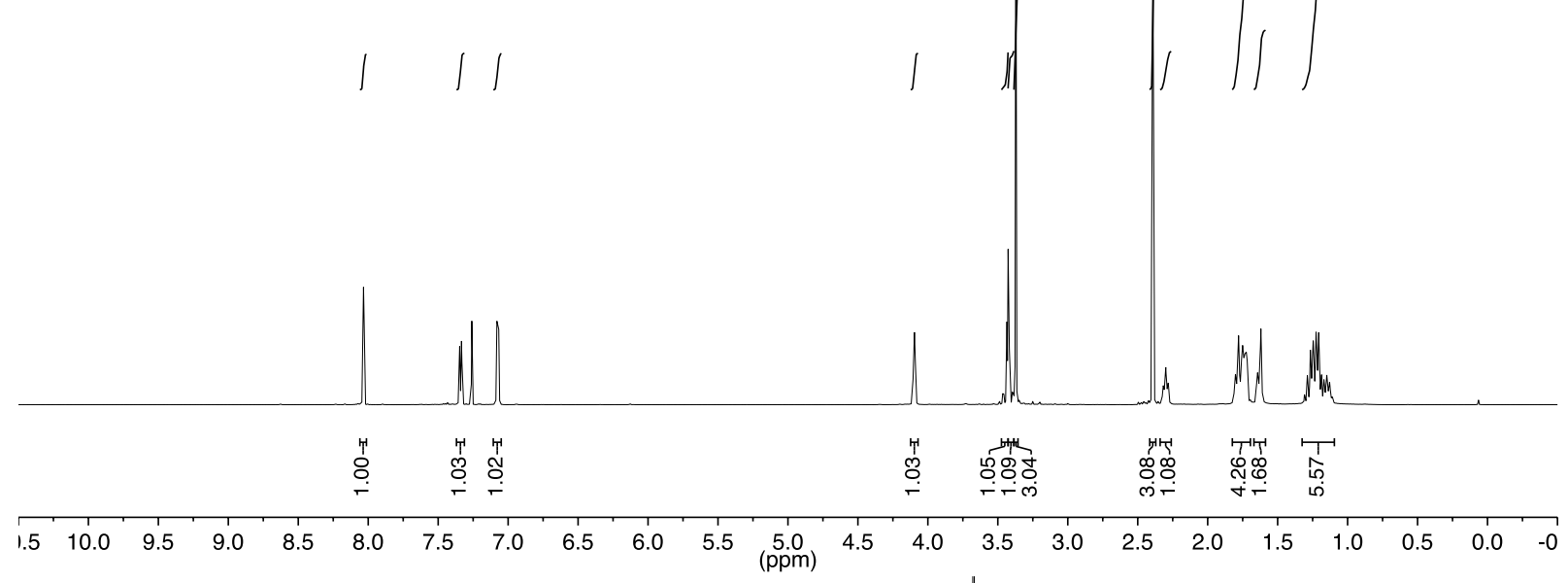

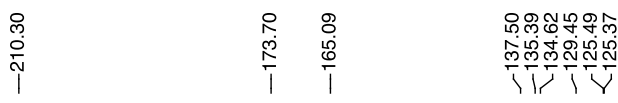

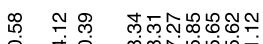

i.

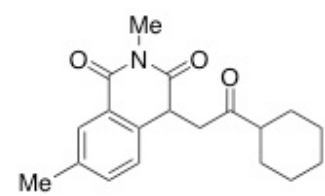

$4 \mathbf{j}$

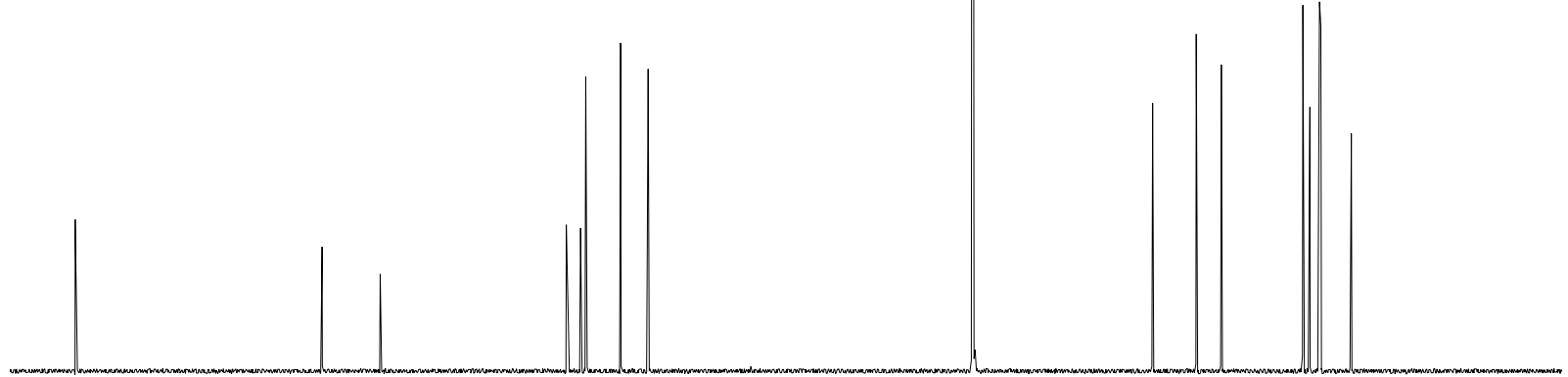

$\begin{array}{lllllllllllllllllllllllllll}20 & 210 & 200 & 190 & 180 & 170 & 160 & 150 & 140 & 130 & 120 & 110 & 100 & 90 & 80 & 70 & 60 & 50 & 40 & 30 & 20 & 10 & 0 & -1\end{array}$ 


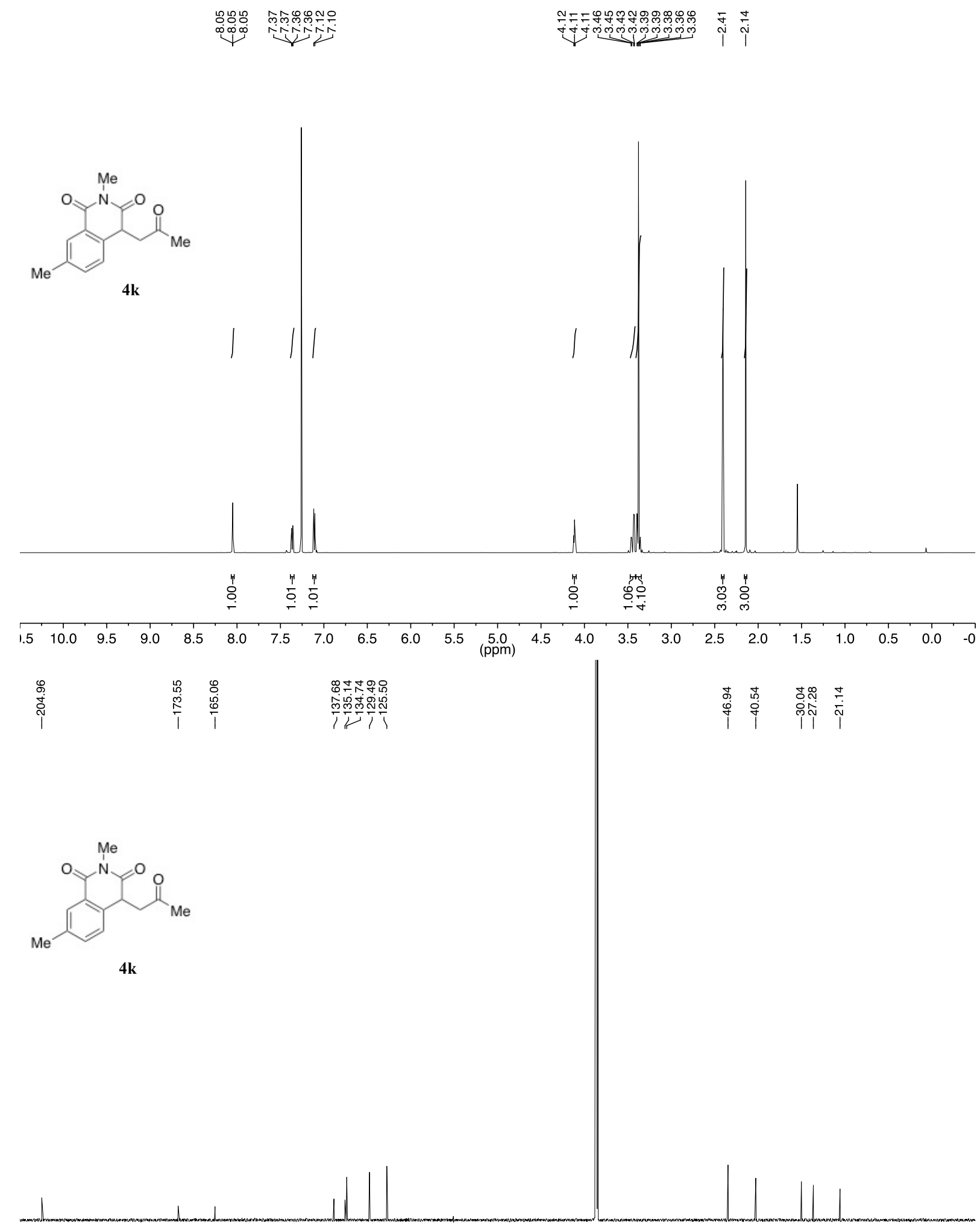

\begin{tabular}{lllllllllllllllllllllll}
\hline 10 & 200 & 190 & 180 & 170 & 160 & 150 & 140 & 130 & 120 & 110 & $\begin{array}{c}100 \\
(\mathrm{ppm})\end{array}$ & 90 & 80 & 70 & 60 & 50 & 40 & 30 & 20 & 10 & 0 & -1
\end{tabular} 


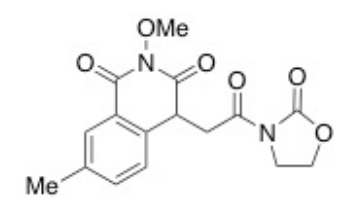

41

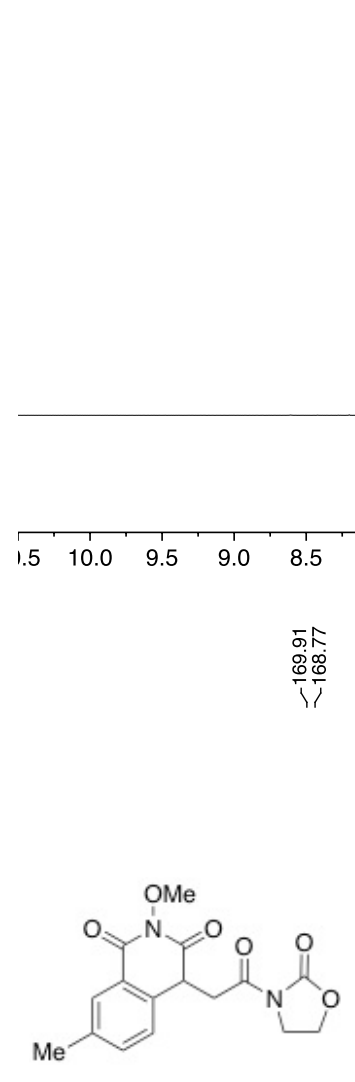

41

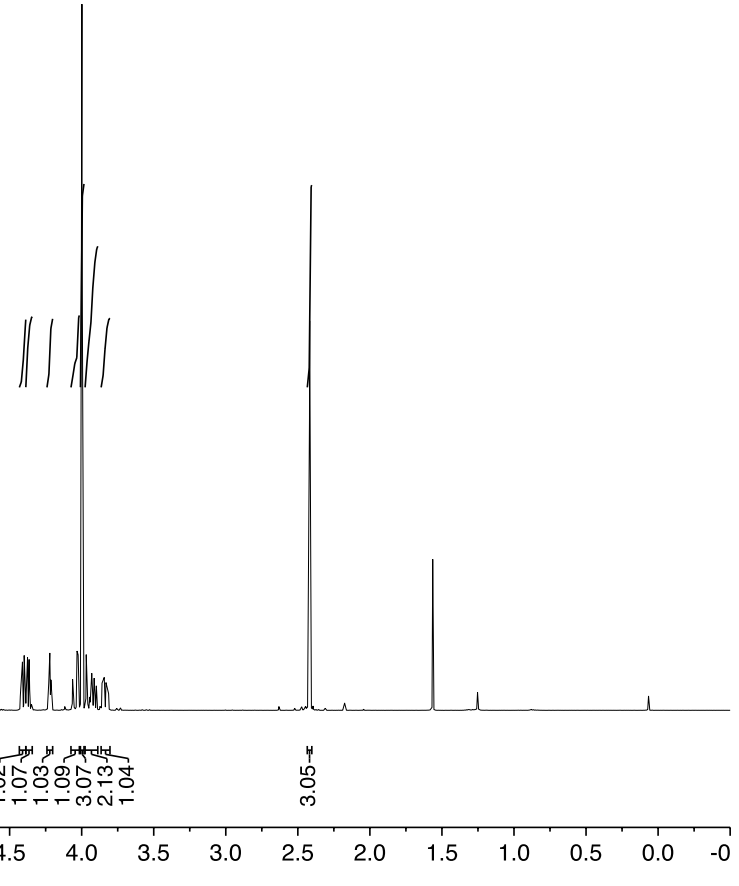

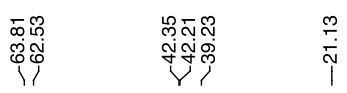

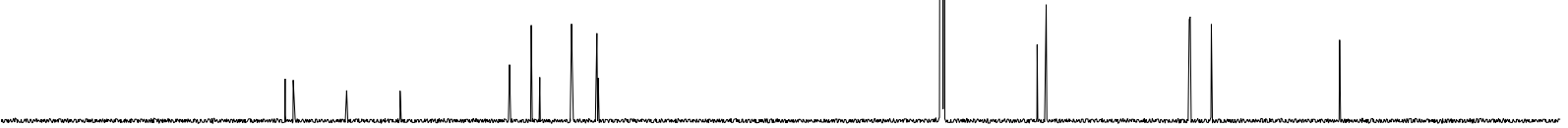

\begin{tabular}{lllllllllllllllllllllllll}
\hline 10 & 200 & 190 & 180 & 170 & 160 & 150 & 140 & 130 & 120 & 110 & $\begin{array}{c}100 \\
(\mathrm{ppm})\end{array}$ & 90 & 80 & 70 & 60 & 50 & 40 & 30 & 20 & 10 & 0 & -1
\end{tabular} 


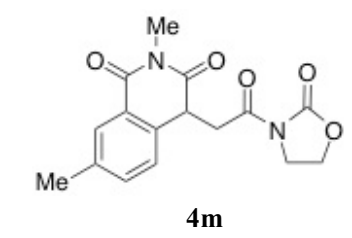

$4 m$

11
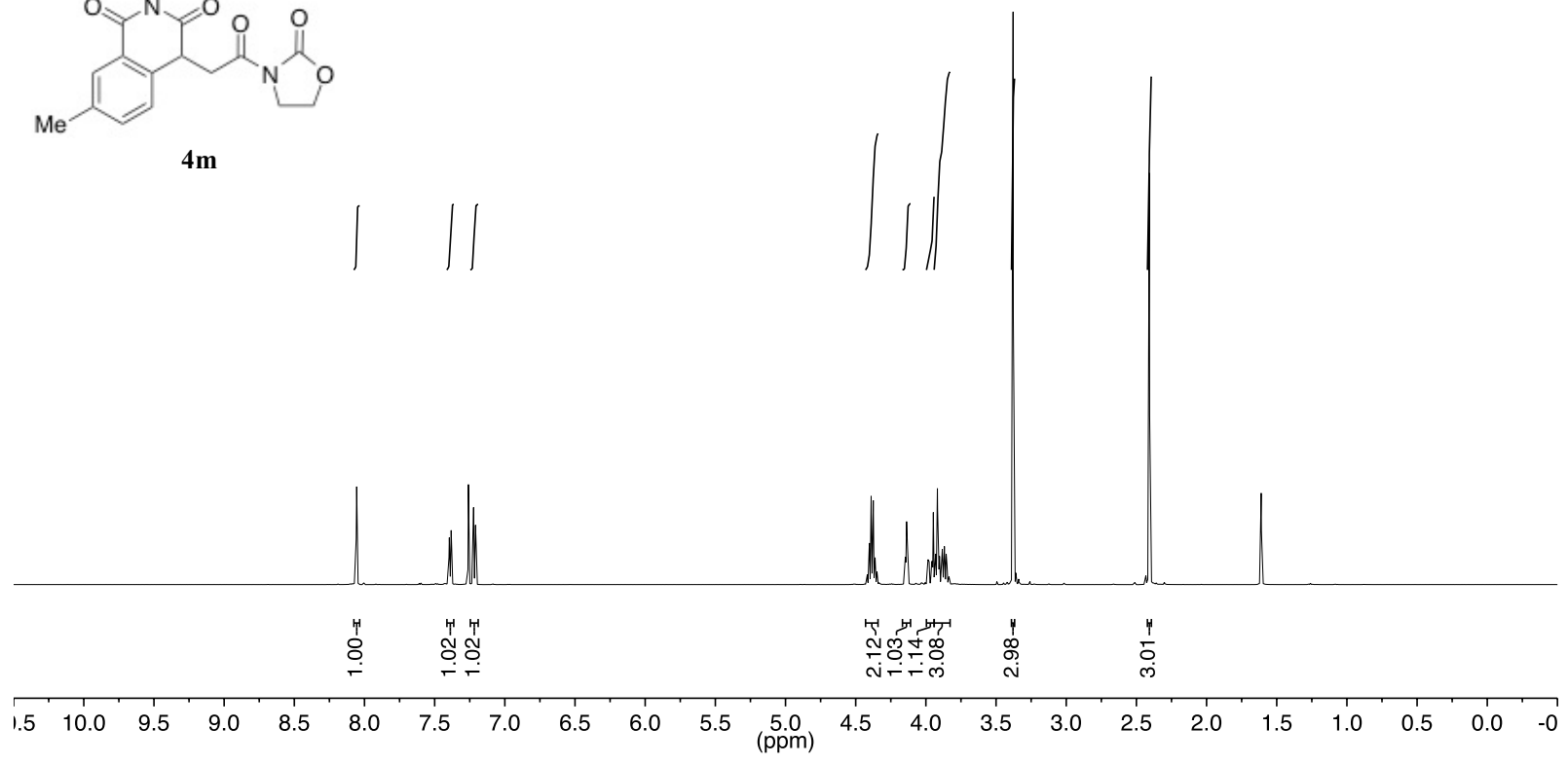

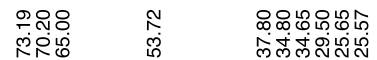

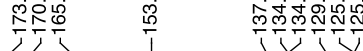

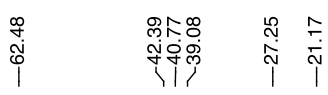
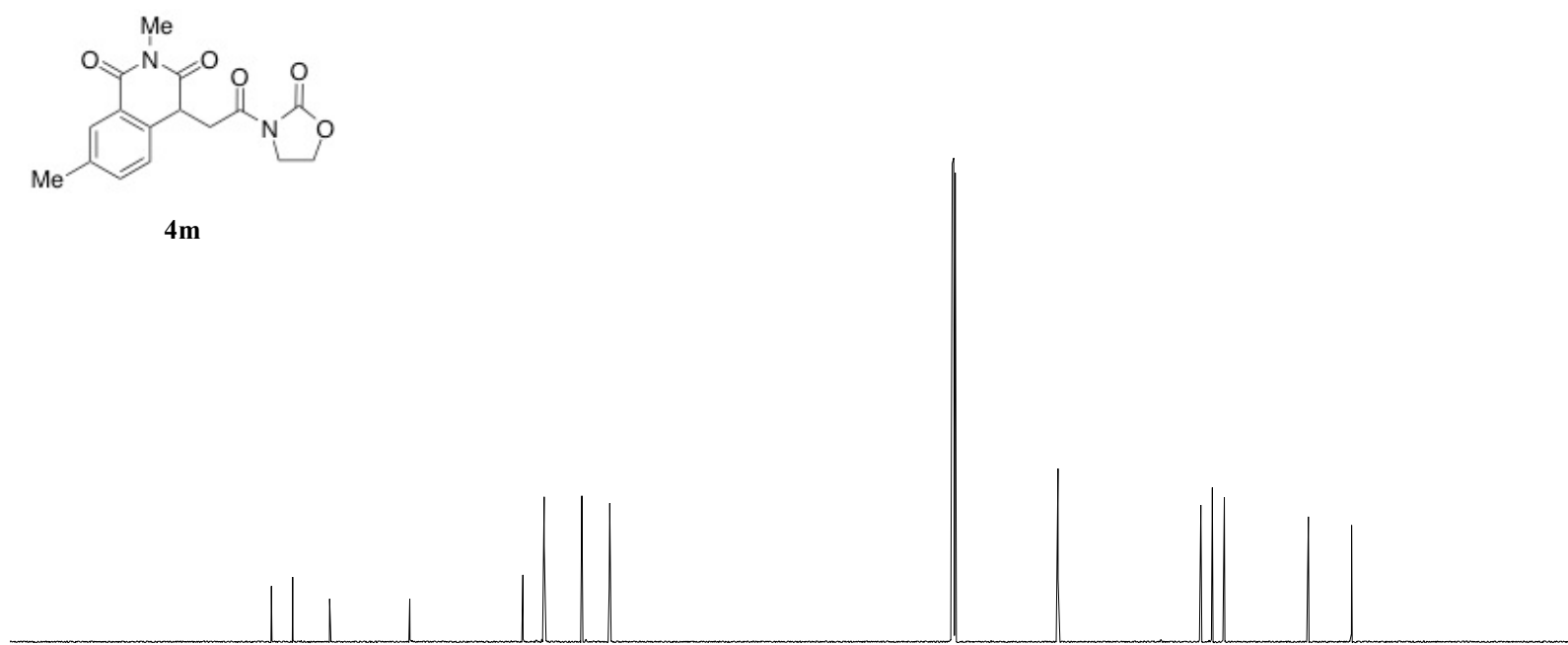

\begin{tabular}{lllllllllllllllllllllll}
\hline 10 & 200 & 190 & 180 & 170 & 160 & 150 & 140 & 130 & 120 & 110 & $\begin{array}{c}100 \\
(\mathrm{ppm})\end{array}$ & 90 & 80 & 70 & 60 & 50 & 40 & 30 & 20 & 10 & 0 & -1
\end{tabular} 

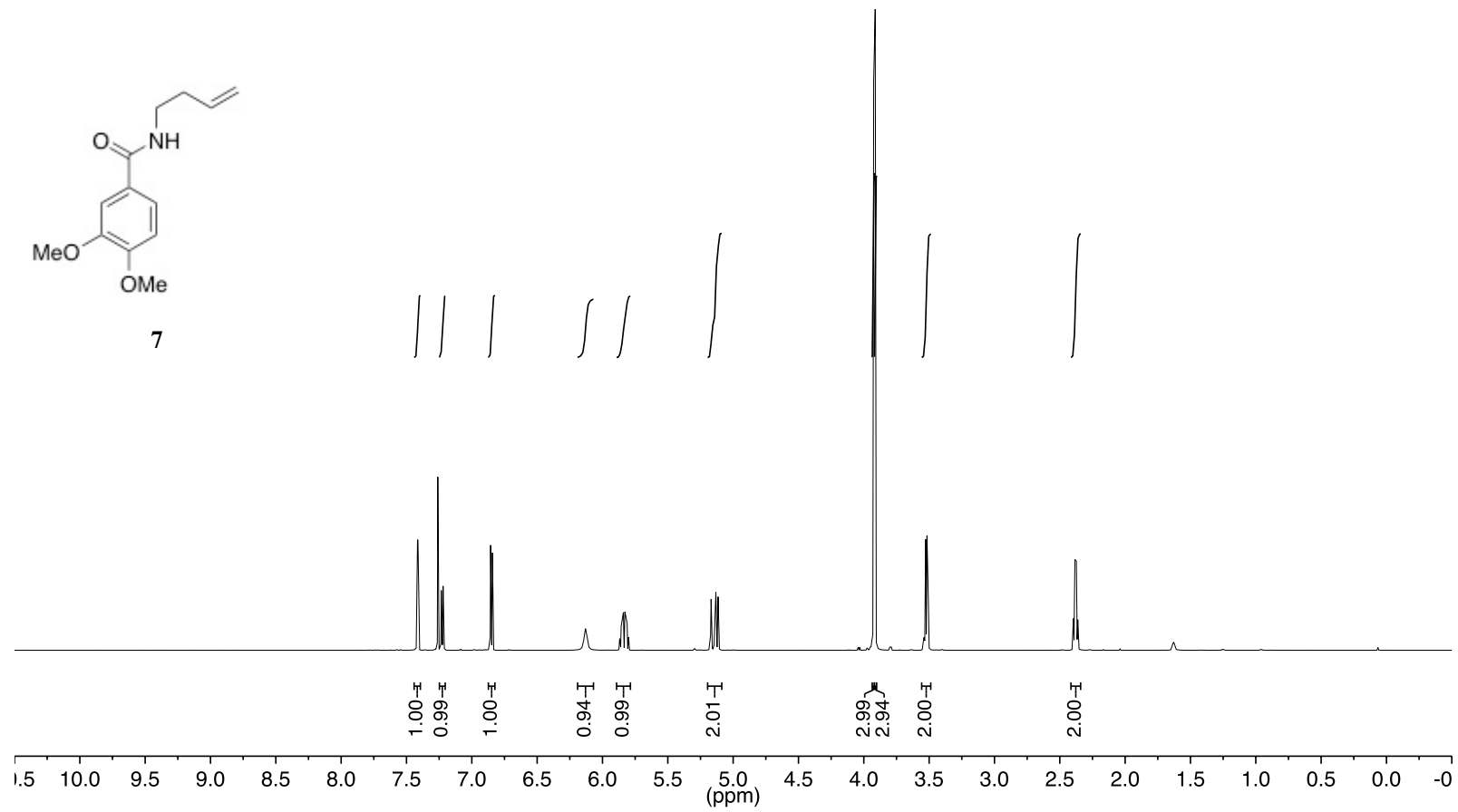

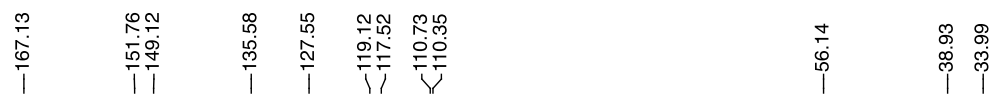

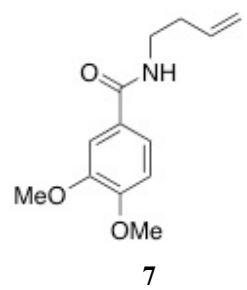

7

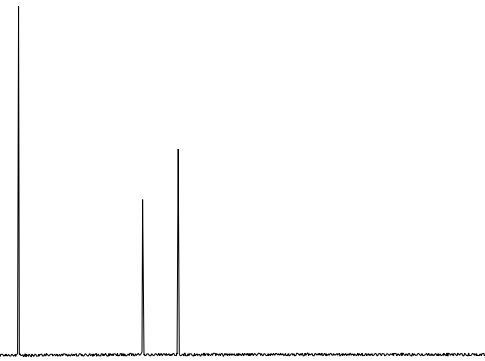

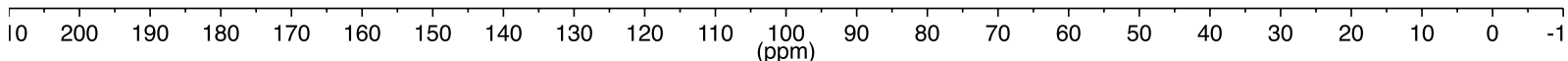



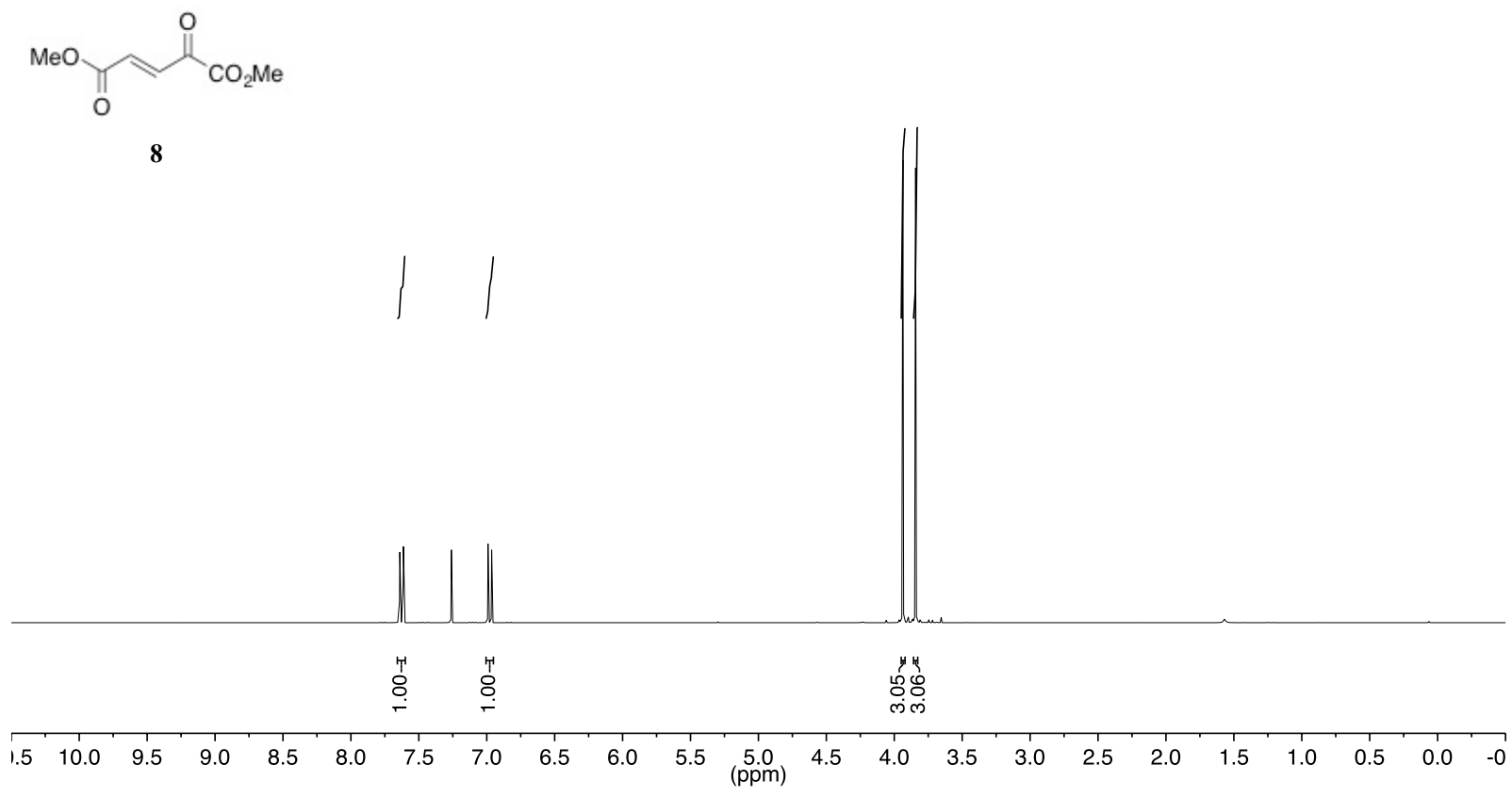

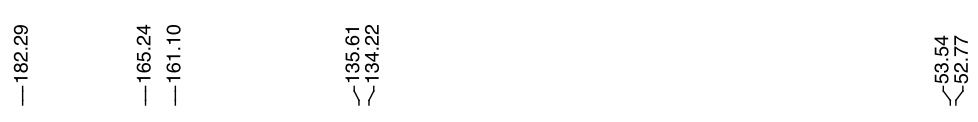
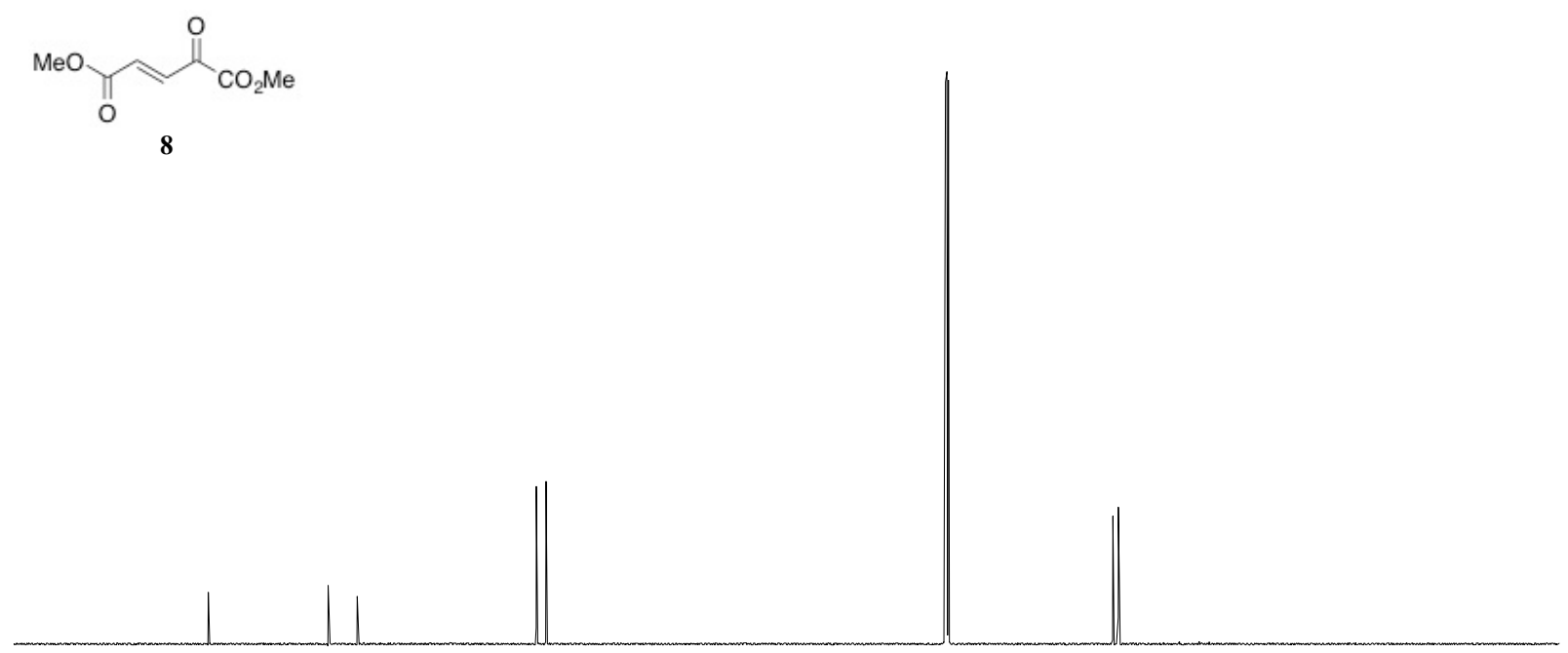

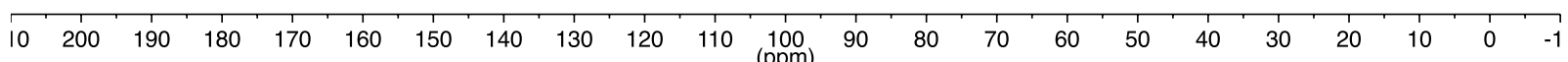




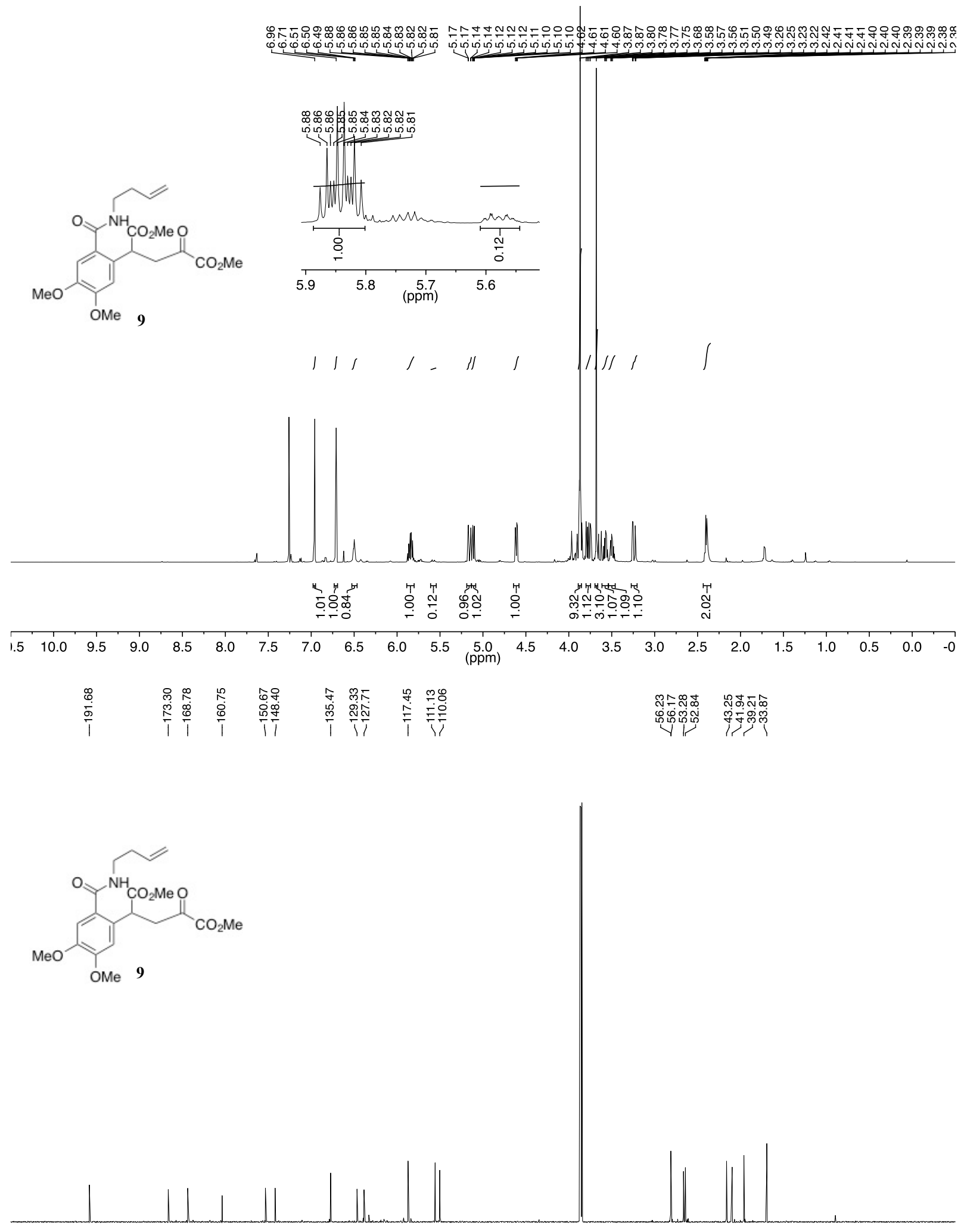

\begin{tabular}{lllllllllllllllllllllll}
\hline 0 & 200 & 190 & 180 & 170 & 160 & 150 & 140 & 130 & 120 & 110 & $\begin{array}{c}100 \\
(\mathrm{ppm})\end{array}$ & 90 & 80 & 70 & 60 & 50 & 40 & 30 & 20 & 10 & 0 & -1
\end{tabular} 


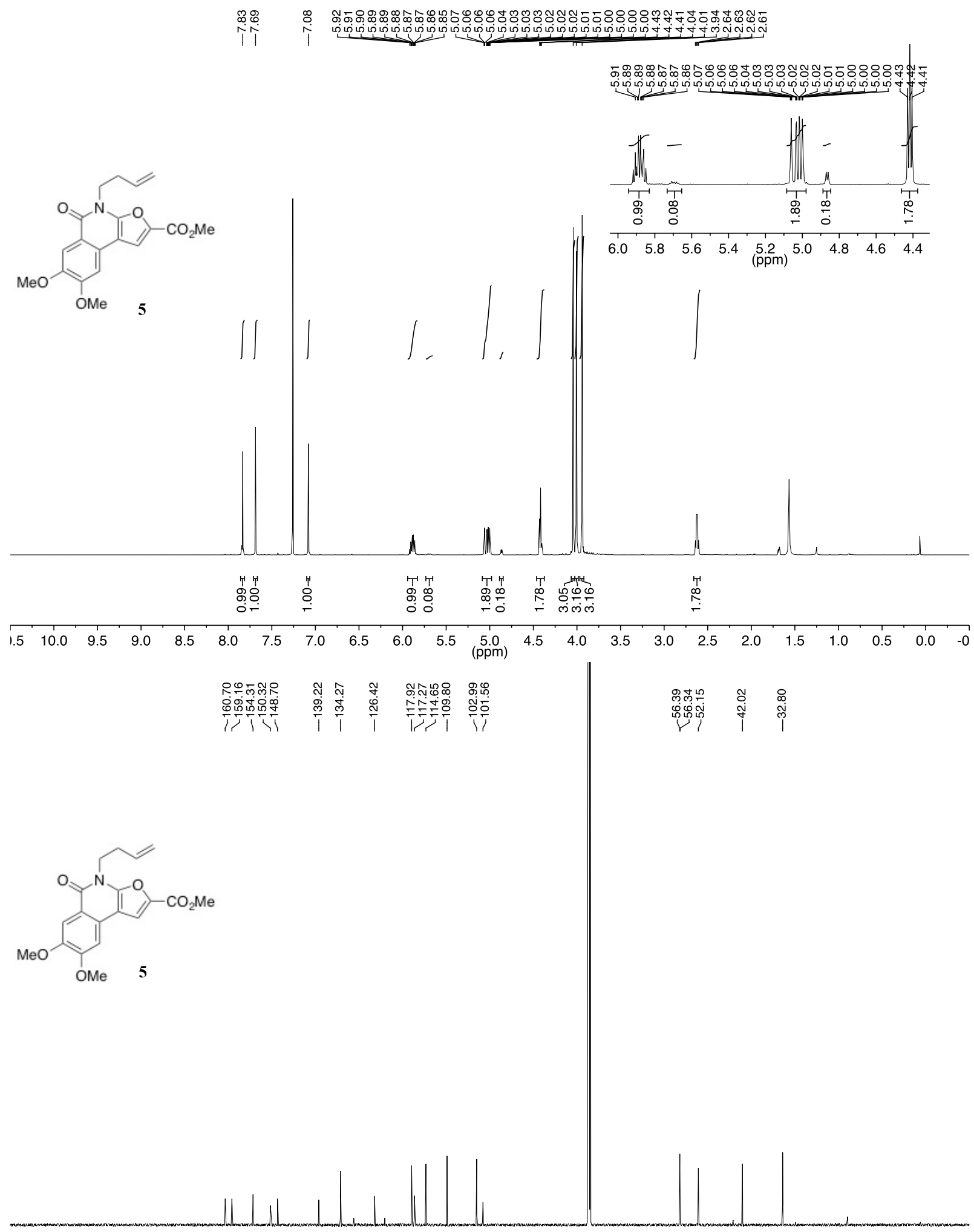

\begin{tabular}{lllllllllllllllllllllll}
\hline 10 & 200 & 190 & 180 & 170 & 160 & 150 & 140 & 130 & 120 & 110 & $\begin{array}{c}100 \\
(\mathrm{ppm})\end{array}$ & 90 & 80 & 70 & 60 & 50 & 40 & 30 & 20 & 10 & 0 & -1
\end{tabular} 


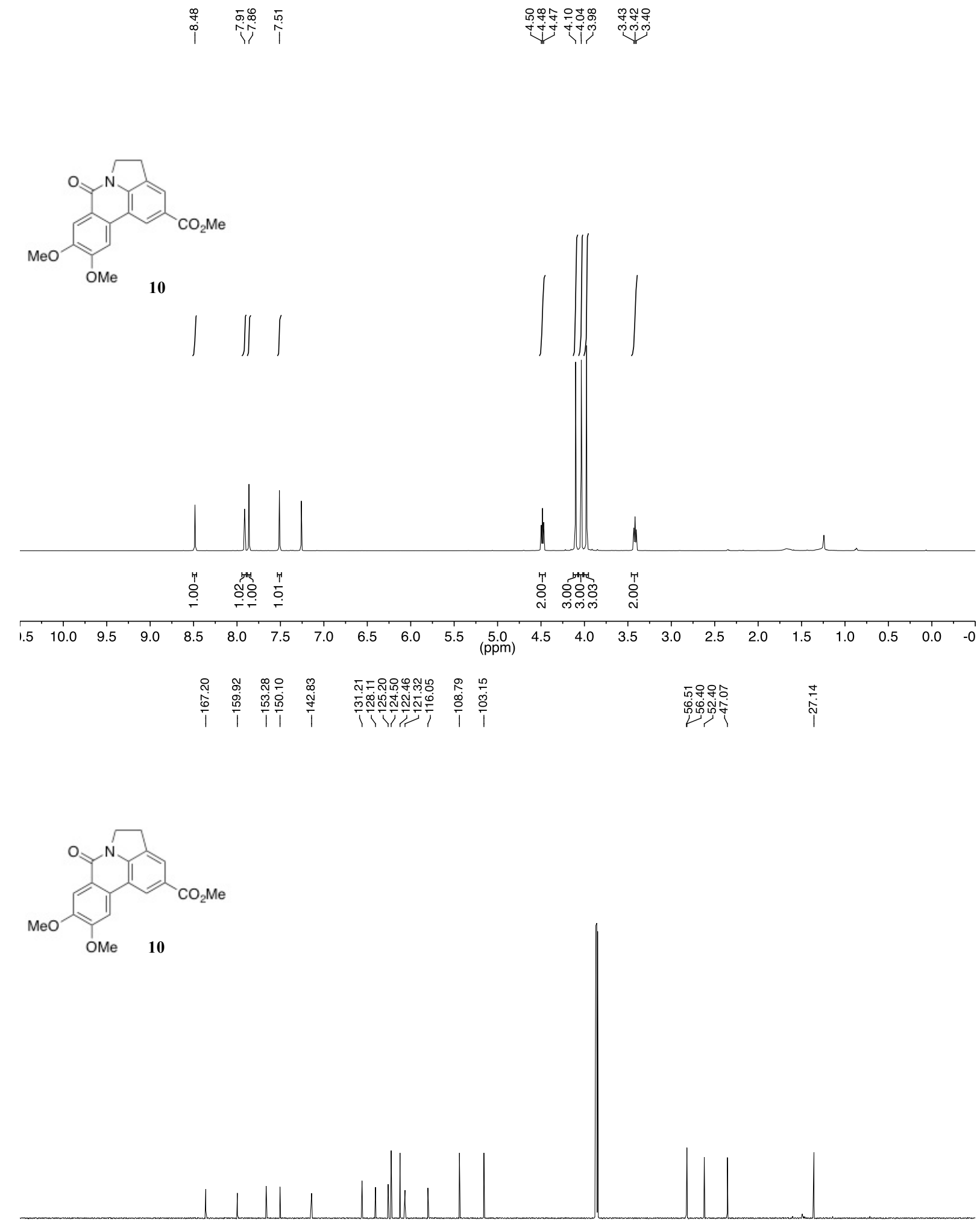

\begin{tabular}{lllllllllllllllllllllll}
\hline 0 & 200 & 190 & 180 & 170 & 160 & 150 & 140 & 130 & 120 & 110 & $\begin{array}{c}100 \\
(\mathrm{ppm})\end{array}$ & 90 & 80 & 70 & 60 & 50 & 40 & 30 & 20 & 10 & 0 & -1
\end{tabular} 


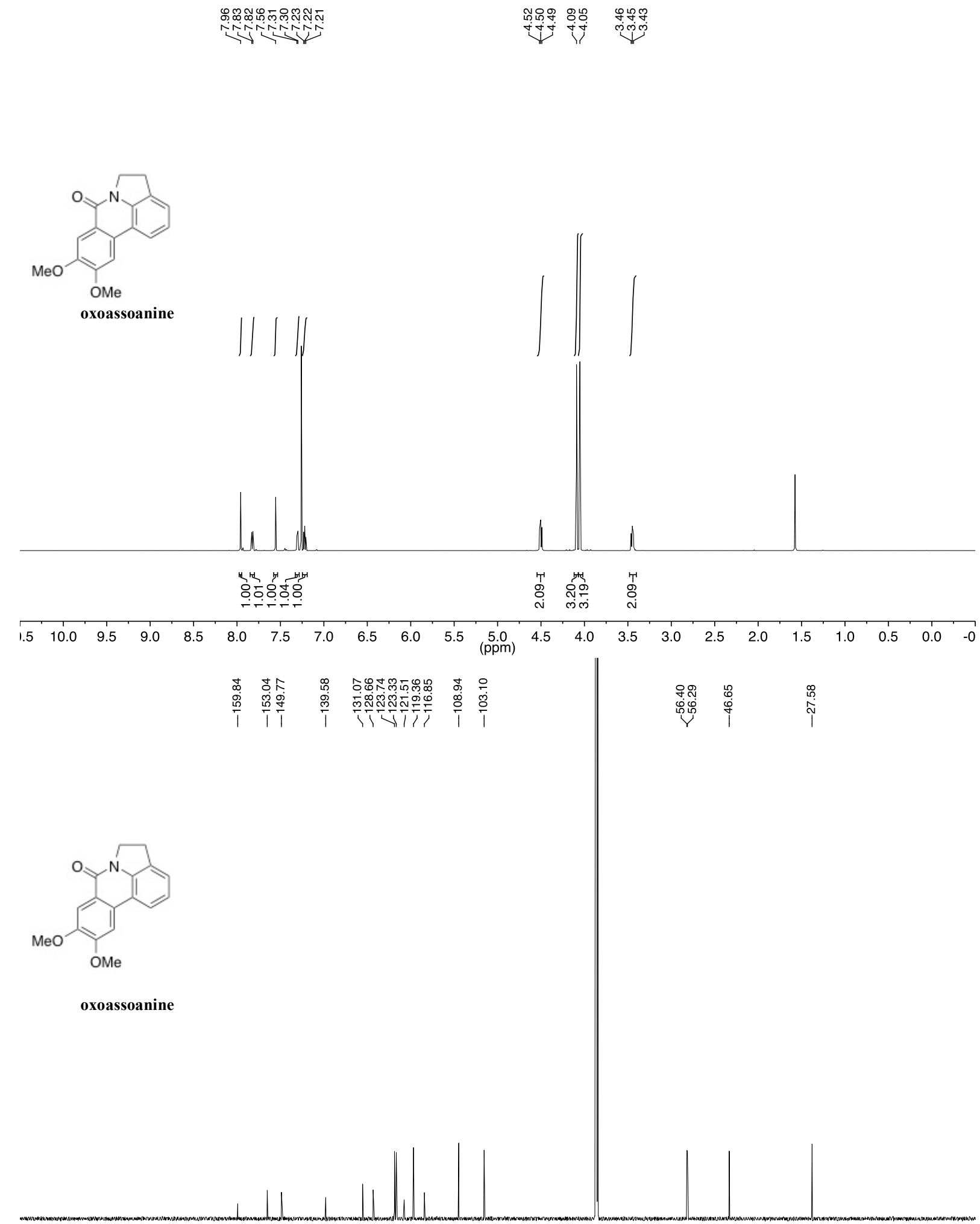

\begin{tabular}{lllllllllllllllllllllll}
\hline 10 & 200 & 190 & 180 & 170 & 160 & 150 & 140 & 130 & 120 & 110 & $\begin{array}{c}100 \\
(\mathrm{ppm})\end{array}$ & 90 & 80 & 70 & 60 & 50 & 40 & 30 & 20 & 10 & 0 & -1
\end{tabular} 